\title{
O teorema de Amitsur para identidades racionais em anéis com divisão
}

\author{
Pedro Russo de Oliveira
}

\author{
DisSERTAÇÃO APRESENTADA \\ $\mathrm{AO}$ \\ Instituto de Matemática e Estatística \\ DA \\ Universidade de SÃo Paulo \\ PARA \\ OBTENÇÃO DO TÍTULO \\ $\mathrm{DE}$ \\ Mestre em CiÊnCIAS
}

Programa: Matemática

Orientador: Prof. Dr. Jairo Zacarias Gonçalves

Durante o desenvolvimento deste trabalho o autor recebeu auxílio financeiro da CAPES

São Paulo, março de 2015 



\section{O teorema de Amitsur para identidades racionais em anéis com divisão}

Esta versão da dissertação contém as correções e alterações sugeridas pela Comissão Julgadora durante a defesa da versão original do trabalho, realizada em 15/05/2015. Uma cópia da versão original está disponível no Instituto de Matemática e Estatística da Universidade de São Paulo.

Comissão Julgadora:

- Prof. Dr. Jairo Zacarias Gonçalves (orientador) - IME-USP

- Prof. Dr. Vitor de Oliveira Ferreira - IME-USP

- Prof. Dr. Daniel Levcovitz - ICMC-USP 



\section{Resumo}

\section{OLIVEIRA, P. R. O teorema de Amitsur para identidades racionais em anéis com}

divisão. 2015. 79 f. Dissertação (Mestrado) - Instituto de Matemática e Estatística, Universidade de São Paulo, São Paulo, 2015.

Sejam $D$ um anel com divisão de centro infinito $K$ e $C$ um subcorpo infinito de $K$. Se $\operatorname{dim}_{K} D=\infty$, provaremos que uma identidade racional (com coeficientes em $C$ ) é válida em $D$ se e somente se é válida em todos os anéis $M_{n}(C)$ de matrizes $n \times n$ sobre $C$, para qualquer $n \geq 1$. Para tais fins, exporemos a teoria de identidades racionais em sua forma original, proposta por Amitsur em [Ami66]. Os resultados obtidos serão usados em duas aplicações. Inicialmente, mostraremos que o grupo multiplicativo de $D$ não satisfaz identidades de grupo não triviais. Em seguida, construiremos um anel com divisão de todas as funções racionais em $D$, o qual denotamos por $C_{D}(x)$, cuja estrutura depende apenas da dimensão de $D$ sobre $K$. Quando $\operatorname{dim}_{K} D=\infty$, vamos mostrar que $C_{D}(x)=C(x)$ pode ser compreendido como um anel universal de frações da álgebra livre com unidade $C\langle x\rangle$ gerada por infinitas indeterminadas não comutativas $x_{1}, x_{2}, \ldots$ Enfatizamos que existe uma versão em língua inglesa do presente trabalho.

Palavras-chave: identidades, identidades racionais, anéis com divisão. 


\section{Abstract}

OLIVEIRA, P. R. Amitsur's theorem for rational identities in division rings. 2015. 79 f. Dissertation (Masters) - Instituto de Matemática e Estatística, Universidade de São Paulo, São Paulo, 2015.

Let $D$ be a division ring with infinite center $K$, and let $C$ be an infinite subfield of $K$. If $\operatorname{dim}_{K} D=\infty$, we shall prove that a rational identity (with coefficients in $C$ ) holds in $D$ if and only if it is a rational identity holding in every ring $M_{n}(C)$ of $n \times n$ matrices over $C$, for all $n \geq 1$. In order to do that, we shall expose the theory of rational identities in its original form, proposed by Amitsur, in [Ami66]. The results we are to obtain will be used in two major applications. Firstly, we will show that the multiplicative group of $D$ does not satisfy a non-trivial group identity. Afterwards, we construct a division ring of all rational functions in $D$, which we denote by $C_{D}(x)$, whose structure depends only on the dimension of $D$ over $K$. When $\operatorname{dim}_{K} D=\infty$, we show that $C_{D}(x)=C(x)$ may be understood as an universal ring of fractions of the free unitary algebra $C\langle x\rangle$ generated by infinite noncommutative indeterminates $x_{1}, x_{2}, \ldots$ We emphasize that there exists an English version of the whole text.

Keywords: identities, rational identities, division rings. 


\section{Sumário}

Algumas notações usadas com freqüência vii

Introdução $\quad$ ix

1 Preliminares 1

1.1 Anéis de frações . . . . . . . . . . . . . . . . . . . . . . . . . 1

1.1.1 O Método de localização de Ore . . . . . . . . . . . . . . . . 1

1.1.2 Anéis de polinômios e extensão de escalares . . . . . . . . . . . . . 4

1.2 Um pouco de topologia . . . . . . . . . . . . . . . . . . . . . . 12

1.3 Filtros e ultra-produtos . . . . . . . . . . . . . . . . . . 13

2 Identidades racionais $\quad 19$

2.1 Identidades Polinomiais . . . . . . . . . . . . . . . . . . . . . . . . 19

2.2 Expressões Racionais . . . . . . . . . . . . . . . . . . . . 20

2.3 Homomorfismos Admissíveis . . . . . . . . . . . . . . . . . . . . . 22

2.4 Extensão de escalares . . . . . . . . . . . . . . . . . . . 26

2.5 Álgebras centrais simples de dimensão finita . . . . . . . . . . . . . . . . . 29

2.6 Álgebras com divisão de dimensão infinita . . . . . . . . . . . . . . . . . . 30

2.7 Um último teorema sobre identidades racionais . . . . . . . . . . . . . . . 40

3 Aplicações $\quad 43$

3.1 O grupo multiplicativo de anéis com divisão . . . . . . . . . . . . . . . . 43

3.2 O anel de funções racionais em um anel com divisão . . . . . . . . . . . . . 48

$\begin{array}{ll}\text { Referências Bibliográficas } & 61\end{array}$

$\begin{array}{ll}\text { Índice Remissivo } & 63\end{array}$ 


\section{Algumas notações usadas com freqüência}

$\begin{array}{ll}\mathbb{Z} & \text { anel dos inteiros } \\ \mathbb{R} & \text { corpo dos reais } \\ C & \text { corpo infinito } \\ D & \text { anel com divisão arbitrário } \\ \mathscr{D}_{m} & \text { álgebra central simples de dimensão } m^{2} \\ \mathfrak{R}=M_{n}(D) & \text { conjunto das matrizes } n \times n \text { com entradas em } D \\ \mathfrak{D}_{\alpha}=\mathfrak{R} \otimes_{C} C(\alpha) & \text { produto tensorial de } \mathfrak{R} \text { com } C(\alpha), \\ & \text { em que } \alpha \text { é algébrico sobre } C \\ \subseteq & \text { inclusão } \\ \subsetneq & \text { inclusão estrita } \\ A \hookrightarrow B & \text { monomorfismo de } A \text { em } B \\ & \text { (alternativamente, } A \text { é subestrutura de } B) \\ \delta_{i j} & \text { delta de Kronecker } \\ E_{i j} & \text { matriz } i j \text {-elementar } \\ \mathfrak{R}[t] & \text { anel de polinômios na indeterminada comutativa } t, \\ & \text { com coeficientes em } \mathfrak{R} \\ \mathfrak{R}(t) & \text { anel total de frações de } \mathfrak{R}[t] \\ S_{\alpha} & \{p[t] \in \mathfrak{R}[t] \mid p[\alpha] \text { é regular em } \mathfrak{R}\} \\ \mathfrak{R}(t) & \text { localização de } \mathfrak{R}[t] \text { com denominadores em } S_{\alpha} \\ \epsilon_{\alpha} & \text { aplicação de } \mathfrak{R} \alpha(t) \text { em } \mathfrak{D}_{\alpha} \text { dada por } a[t] b[t]^{-1} \mapsto a[\alpha] b[\alpha]^{-1} \\ \mathscr{F} & \text { filtro } \\ C_{0}=C\langle x\rangle & C \text {-álgebra associativa, livre e unitária, nos geradores } \\ C_{n}=C\langle x ; y\rangle & \{x\}=\left\{x_{1}, x_{2}, \ldots\right\} \\ \mathfrak{I}(D) & C \text {-álgebra associativa, livre e unitária, nos geradores } \\ I(D) & \{x\}=\left\{x_{1}, x_{2}, \ldots\right\} \text { e }\{y\}=\left\{y_{1}, \ldots, y_{n}\right\} \\ \mathfrak{F}[\mathfrak{F}] & \text { conjunto dos homomorfismos admissíveis de } C\langle x ; y\rangle \text { em } D \\ & \text { conjunto (ideal) de identidades racionais de } D \\ & \text { grupo livre gerado por }\{\xi\}=\left\{\xi_{1}, \xi_{2}, \ldots\right\} \\ & C \text {-álge de } \mathfrak{F} \text { sobre } C \\ & \end{array}$




\section{Introdução}

O estudo de identidades na teoria de anéis começou com Dehn [Deh22], com a pretensão - que veio a fracassar - de resolver problemas ligados à área de geometria projetiva, usando identidades polinomiais. A relevância do estudo de relações polinomiais em álgebras veio a se estabelecer, de maneira definitiva, após trabalhos de Jacobson [Jac45] - sobre o problema de Kurosch [Kur41] - e de Kaplansky [Kap48], anos mais tarde. Um sem-número de publicações relacionadas ao tema veio logo em seguida, entre as quais destacamos um material conjunto de Amitsur e de Levitski, em [AL50]. Mais à frente no tempo, Amitsur, em [Ami65], classificou o que se havia desenvolvido até então como a teoria clássica das relações polinomiais e deu um passo à frente, estabelecendo o conceito de identidades polinomiais generalizadas. Nesse artigo, o autor utiliza ultra-produtos, os quais desde já se revelam bastante eficazes, para mostrar que a álgebra dos polinômios generalizados sobre um anel com divisão $D$ arbitrário pode ser embutida em um anel com divisão construído a partir de $D$. Um ano depois, Amitsur publica, pioneiramente, uma teoria de identidades racionais em [Ami66], como uma alternativa às polinomiais para sedimentar - desta vez, com êxito - uma solução para os problemas que ocuparam Dehn.

A abordagem original de Amitsur não tardou a ser burilada e generalizada, conforme Bergman, em [Ber70], [Ber76a] e [Ber76b], cujos trabalhos são tratados por Rowen, em [Row80], capítulo 8. Mais recentemente, encontramos, na literatura, tópicos dedicados a identidades racionais, elaborados de maneira mais simples e geral que a pioneira, como em muitos materiais de Cohn, por exemplo [Coh95], capítulo 7. Em [Ber76a] (nota de rodapé, p.258), Bergman fala sobre identidades racionais em anéis com divisão de centros finitos. Até hoje, carecemos de resultados conclusivos sobre o assunto.

A noção de identidade racional é anterior ao estabelecimento da teoria por Amitsur em [Ami66]. Grosso modo, uma identidade racional é uma expressão em indeterminadas que envolve inversos multiplicativos e, para quaisquer substituições (com respeito à existência de inversos) em uma dada álgebra, tal expressão é identicamente nula. Um exemplo vastamente explorado, o qual trataremos com mais cuidado adiante, é a identidade de Hua (e.g. Rowen [Row80], p. 290):

$$
a-\left[a^{-1}+\left(b^{-1}-a\right)^{-1}\right]^{-1}-a b a=0 .
$$

Embora uma primeira olhada não revele, esta é uma identidade racional de qualquer álgebra em que estejam definidos $a^{-1}, b^{-1},\left(b^{-1}-a\right)^{-1}$ e $\left[a^{-1}+\left(b^{-1}-a\right)^{-1}\right]^{-1}$. Em 
outras palavras, podemos afirmar, por exemplo, que se trata de uma identidade trivial na classe dos anéis com divisão, pois não provoca impacto algum sobre a classe, ou seja, não determina uma subclasse própria. Isso contrasta com as identidades polinomiais: um anel com divisão que satisfaz uma identidade polinomial tem, necessariamente, dimensão finita sobre seu centro (cf. Kaplansky [Kap48]). Não obstante, o fato de uma identidade racional ser trivial não lhe nubla a importância: a identidade de Hua é aplicada na demonstração do teorema fundamental da geometria projetiva.

Como não dispomos de algoritmos para julgar se uma identidade racional em um anel com divisão é ou não trivial, a pergunta natural, a esta altura, é: existem identidades racionais não triviais em anéis com divisão? Uma resposta satisfatória vem em [Ami66]. O objetivo do nosso texto é tratar a teoria das identidades racionais e algumas de suas aplicações, da maneira originalmente proposta por Amitsur. Nosso material será estruturado em três capítulos.

Todo primeiro capítulo pode sempre ser maior, abrangendo mais e mais fatos elementares, ao ponto de extrapolar o escopo do trabalho. Para evitar isso, o nosso primeiro capítulo é um breve mosaico de tópicos preliminares, os quais julgamos essenciais aos seguintes. Exporemos de maneira sumária alguns resultados sobre anéis de frações, no sentido de Ore [Ore31], sobremaneira aqueles obtidos a partir de álgebras de polinômios. Dedicamos especial atenção à seção que trata de filtros e de ultra-produtos. Estes são usados extensivamente durante o texto para embutir uma álgebra sem divisores de zero em uma com divisão.

O segundo - e o mais importante - capítulo traz a teoria das identidades racionais. Vamos trabalhar com um corpo infinito $C$ e mostrar que as identidades racionais de $C$ álgebras centrais simples de dimensão finita $n^{2}$ são precisamente as do anel de matrizes $M_{n}(C)$. Em seguida, provaremos o resultado principal: o conjunto $I(D)$ das identidades racionais de uma $C$-álgebra $D$ com divisão de dimensão infinita sobre seu centro é, precisamente, $I(D)=\bigcap I\left(M_{n}(D)\right)$, ou seja, o de todas as identidades racionais comuns a todos os anéis de matrizes sobre $C$. Destacamos alguns passos importantes no curso deste capítulo:

- As identidades de uma $C$-álgebra $D$ com divisão são as mesmas de $D(t)$, o anel total de frações obtido a partir do anel de polinômios $D[t]$. A fortiori, isso será usado para mostrar que o conjunto de identidades racinais é invariante sob extensão de escalares.

- O conjunto das identidades racionais do anel com divisão $D((t))$ das séries formais de Laurent com coeficientes em $D$ é, ainda, o mesmo de $D(t)$, portanto o mesmo de $D$.

- Dadas $D$ e $E C$-álgebras com divisão tais que $D \subseteq E$ e a dimensão de $D$ sobre seu centro é infinita, então as identidades racionais de ambas são as mesmas. 
Por fim, dada uma $C$-álgebra $D$ com divisão de dimensão infinita sobre seu centro, vamos construir $C$-álgebra com divisão $\mathscr{D}$ também de dimensão infinita sobre seu centro, cujas identidades racionais são todas aquelas satisfeitas por todos os anéis de matrizes sobre $C$, e ainda uma álgebra $E$ que contém ambas $D$ e $\mathscr{D}$. O resultado principal seguirá com auxílio do último passo listado acima.

Reservamos o terceiro capítulo para tratar de duas aplicações importantes. Na primeira seção, vamos embutir a $C$-álgebra de grupo $C[\mathfrak{F}]$, em que $\mathfrak{F}$ é um grupo livre de infinitos geradores, em um anel com divisão de dimensão infinita sobre seu centro. Usaremos isso para mostrar que o grupo multiplicativo de uma $C$-álgebra com divisão de dimensão infinita não verifica identidade de grupo alguma. Em particular, o grupo multiplicativo de uma álgebra com divisão, nessas condições, não pode ser solúvel. Na segunda seção, vamos construir um anel com divisão $C_{D}(x)$ de funções racionais na $C$ álgebra com divisão $D$, a partir da álgebra livre com unidade $C\langle x\rangle$. Usaremos os resultados sobre identidades racionais para mostrar que tal construção depende apenas da dimensão de $D$ sobre seu centro: no caso finito, $C_{D}(x)$ é uma álgebra central simples de dimensão finita (igual à dimensão de $D$ ). Por outro lado, se ambas $D$ e $D^{\prime}$ têm dimensão infinita, então $C_{D}(x)$ e $C_{D^{\prime}}(x)$ são naturalmente isomorfos. Denotaremos, neste último caso, $C(x)$ e vamos ainda mostrar que se trata de um anel universal de frações da álgebra livre $C\langle x\rangle$. Enfatizamos que, embora ainda não exista um tratamento para identidades racionais em anéis com divisão de centro finito, é possível empregar um método alternativo (e.g. Cohn [Coh95], capítulo 6) para construir um anel universal de frações para $C\langle x\rangle$, mesmo quando $C$ é um corpo finito.

Apesar de o destaque ser dado aos principais resultados, este trabalho envolve outros menores, mas também importantes para o estudo de anéis com divisão, como, por exemplo, os meios pelos quais produzem-se novos anéis com divisão, sobretudo ultra-produtos e o método de Ore. Esperamos que seja proveitoso ao leitor o contato com a teoria em sua forma original, perfazendo lemas, proposições, definições e observações envolvidos, e que o inspire não só a explorar o tema, mas também a transcender os limites do conhecimento em álgebra. Por termos estas expectativas, procuramos analisar o nosso material, sempre que possível, em fatos mais elementares, próximos daqueles relacionados com os tópicos que compõem a formação dos que lidam com álgebra. 



\section{Capítulo 1}

\section{Preliminares}

O resultado principal do nosso trabalho é concernente a anéis com divisão de centro infinito arbitrários. Ainda assim, devemos apresentar, a partir de agora, algumas estruturas algébricas - sobremaneira novos anéis com divisão construídos a partir de um anel com divisão dado inicialmente - que desempenham papel coadjuvante por todo o caminho que traçaremos para atingir nossos objetivos. Este capítulo estabelece uma base mínima para a compreensão do texto global, porquanto é dedicado àquelas pequenas peças que se entrelaçam e originam argumentos robustos e importantes no nosso estudo. Ele provê uma exposição de alguns resultados importantes que serão usados nos próximos capítulos. Como o nosso trabalho não é um material auto-contido, espera-se que o leitor possua considerável conhecimento de estruturas algébricas elementares, homomorfismos e os teoremas a eles relacionados (indicamos Jacobson, [Jac85] e [Jac89]), e de tópicos em teoria de corpos (indicamos Kaplansky, [Kap72]).

Todos os anéis (álgebras) com os quais trabalharemos possuem um elemento identidade, genericamente denotado por 1 . Usaremos o símbolo $\hookrightarrow$ de maneira indistinta para monomorfismos e para denotar subanéis (subálgebras). Convencionamos, ainda, que a identidade de um subanel é a mesma do anel, de modo que será consistente também convencionar que homomorfismos preservam elementos identidade.

\subsection{Anéis de frações}

\subsubsection{O Método de localização de Ore}

O método usualmente empregado para embutir um dado anel (sem divisores de zero não triviais) em um anel com divisão consiste em construir inversos para os elementos não nulos no dado anel. Isso pode ser alcançado de maneira descrita pioneiramente por Ore [Ore31]. Os próximos resultados relacionados ao método de Ore, cujas demonstrações podem ser encontradas em [Lam99], capítulo 4, por exemplo, serão enunciados de maneira mais geral e, em seguida, faremos a restrição deles aos nossos fins.

Em um anel arbitrário $R$, um subconjunto $S \subseteq R$ é dito multiplicativo se $1 \in S$, 
$0 \notin S$ e se $S$ é fechado para a multiplicação. Um homomorfismo de anéis $\psi: R \rightarrow R^{\prime}$ é dito $S$-invertível se $\psi(S) \subseteq \mathcal{U}\left(R^{\prime}\right)$, em que este último é o grupo das unidades de $R^{\prime}$. No que seguirá, $S \subseteq R$ denota um subconjunto multiplicativo de $R$.

Definição 1.1.1. Dados um anel $R^{\prime}$ e um homomorfismo $\psi: R \rightarrow R^{\prime}$, o par $\left(R^{\prime}, \psi\right)$ é dito uma localização (à direita) de $R$ com respeito a $S$ se são verificadas as três seguintes condições:

L1 $\psi$ é S-invertível.

L2 Para todo $x$ em $R^{\prime}$, existem a em $R$ e b em $S$ tais que $x=\psi(a)[\psi(b)]^{-1}$.

L3 $\operatorname{ker} \psi=\{a \in R \mid \exists s \in S$ tal que as $=0\}$.

Neste caso, dizemos que $R$ admite uma localização (à direita) $R^{\prime}$ com respeito a $S$.

Definição 1.1.2. S é dito um conjunto de denominadores (à direita) se ambas as condições abaixo são verificadas:

D1 Quaisquer $s$ em $S$ e r em $R$ verificam $s R \cap r S \neq \emptyset$

D2 Dado $r$ em $R$, se $s r=0$ acontece para algum s em $S$, então existe $s^{\prime}$ em $S$ tal que $r s^{\prime}=0$.

D1 é conhecida como condição de Ore (à direita). Se $S$ satisfizer D1, então dizemos que é um conjunto de Ore. Os resultados que seguirão estabelecem ambas existência e unicidade, no sentido de uma propriedade universal, de localizações.

Teorema 1.1.3. Um anel $R$ admite uma localização com respeito a $S$ se e somente se este é um conjunto de denominadores.

Lema 1.1.4. Dada uma localização $\left(R^{\prime}, \psi\right)$ de $R$ com respeito a $S$, se um homomorfismo $\varphi: R \rightarrow R^{\prime \prime}$ verifica $\mathbf{L} \mathbf{1}$, então existe um único homomorfismo $\eta: R^{\prime} \rightarrow R^{\prime \prime}$ tal que $\eta \psi=\varphi$.

Corolário 1.1.5. Em termos do lema acima, se $\varphi$ é um monomorfismo, então $\eta$ também $o$ é.

Prova: Dado $x \in \operatorname{ker} \eta \subseteq R^{\prime}$, devem existir $a \in R$ e $b \in S$ tais que $x=\psi(a)[\psi(b)]^{-1}$. Logo $0=\eta(x)=\eta\left(\psi(a)[\psi(b)]^{-1}\right)=(\eta \psi)(a)[(\eta \psi)(b)]^{-1}=\varphi(a)[\varphi(b)]^{-1}$. Como $[\varphi(b)]^{-1}$ é uma unidade em $R^{\prime \prime}$, ocorre $\varphi(a)=0$. Por hipótese, resulta que $a=0$, o que nos conduz à conclusão final $x=0$, completando esta prova.

Ilustramos o corolário 1.1.5 no seguinte diagrama comutativo:

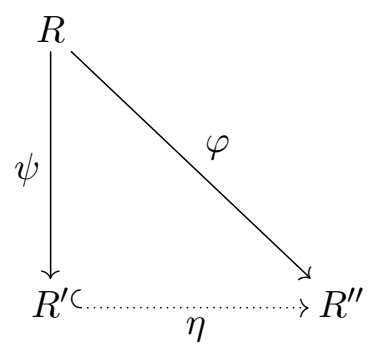


Teorema 1.1.6. Se $\left(R^{\prime}, \psi\right)$ e $\left(R^{\prime \prime}, \varphi\right)$ são localizações de $R$ com respeito a $S$, então existe um único isomorfismo $\eta: R^{\prime} \rightarrow R^{\prime \prime}$ tal que $\eta \psi=\varphi$.

Prova: Temos o seguinte diagrama comutativo:

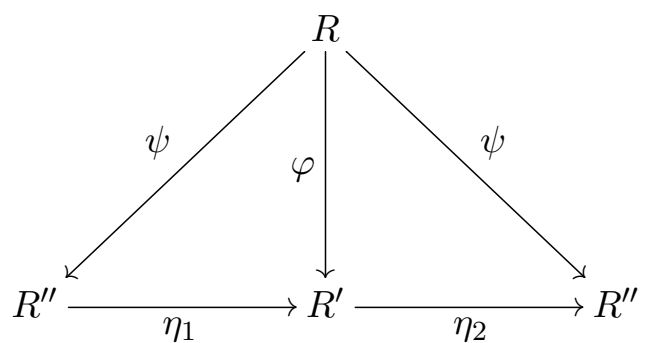

De acordo com o lema 1.1.4, $\eta_{1}$ e $\eta_{2}$ são os únicos homomorfismos que verificam

$$
\eta_{1} \psi=\varphi \text { e } \eta_{2} \varphi=\psi
$$

do que sucede

$$
\eta_{2} \eta_{1} \psi=\psi \text { e } \eta_{1} \eta_{2} \varphi=\varphi
$$

Mais uma vez pelo lema 1.1.4, as aplicações identidade $i d^{\prime}$ e $i d^{\prime \prime}$ de $R^{\prime}$ e de $R^{\prime \prime}$, respectivamente, são as únicas que verificam $i d^{\prime} \varphi=\varphi$ e $i d^{\prime \prime} \psi=\psi$. Concluímos, assim, que

$$
\eta_{1} \eta_{2}=i d^{\prime} \text { e } \eta_{2} \eta_{1}=i d^{\prime \prime}
$$

o que encerra esta prova.

À luz do precedente teorema, faz sentido denotar por $\left(R_{S}, \psi_{S}\right)$ a localização de $R$ com respeito a $S$. Devemos, agora, particularizar os resultados acima aos nossos propósitos. Introduzimos a próxima

Definição 1.1.7. Um elemento em um anel $R$ é dito regular se não é um divisor de zero nem à direita, nem à esquerda em $R$.

Como vamos trabalhar apenas com conjuntos multiplicativos $S$ compostos de elementos regulares, a condição D2 é imediatamente verificada. Por conseguinte, a fim de mostrar que $S$ é um conjunto de denominadores, restará validar D1. Sendo isso verdade, decorre de $\mathbf{L} 3$ que $\operatorname{ker} \psi_{S}=(0), \operatorname{logo} R$ é isomorfo a um subanel de $R_{S}$. Em virtude desta última observação, para sermos breves, podemos abandonar em segurança o monomorfismo $\psi_{S}$ e identificar diretamente $R$ como um subanel de $R_{S}$, cujos elementos, daqui em diante, serão descritos em termos da condição L2. Faremos referência a $R_{S}$ como o anel de frações (à direita, com denominadores em $S$ ) de $R$. No caso especial em que $S=\{r \in R \mid r$ é regular em $R\}$ é um conjunto de Ore, $R$ é dito ser um anel de Ore e $R_{S}$ é dito o anel total de frações de $R$.

Definição 1.1.8. Um anel (não necessariamente comutativo) é dito ser um dominio se todos os seus elementos não nulos são regulares. 
Destacamos que, se um domínio é um anel de Ore, então o seu anel total de frações é um anel com divisão, porquanto os seus elementos não nulos são unidades. A próxima proposição estabelece uma útil conexão entre o método de Ore e a condição de cadeia ascendente para ideais.

Proposição 1.1.9. Seja $R$ um domínio noetheriano (à direita). Então $R$ é um anel de Ore e está embutido em um anel com divisão, a saber, o seu anel total de frações.

Prova: Suponhamos que $R$ seja um domínio noetheriano (à direita). Resta mostrar que o conjunto multiplicativo $R^{*}$ dos elementos não nulos em $R$ é um conjunto de Ore. Dados $a \in R^{*}$ e $b \in R$, se $b=0$ o resultado segue prontamente. Se também $b$ é não nulo, o ideal à direita gerado pelos elementos não nulos $b, a b, a^{2} b, \ldots$ deve ser finitamente gerado, portanto os listados geradores devem ser linearmente dependentes à direita sobre $R$. Seja $\sum_{i=0}^{n} a^{i} b c_{i}=0$ uma equação de dependência linear, em que os $c_{i}$ 's não são todos nulos. Seja $k \in\{0, \ldots, n\}$ o primeiro índice tal que $c_{k} \neq 0$. Como $R$ é um domínio, $k<n$ e podemos cancelar $a^{k}$, o que implica $b c_{k}+a b c_{k+1}+\cdots+a^{n-k} b c_{n}=0$. Logo $a\left(b c_{k+1}+\cdots+a^{n-k-1} b c_{n}\right)=-b c_{k} \neq 0$ é um múltiplo comum de $a$ e $b$, o que valida D1 para $R$, como queríamos.

Claramente, tudo o que expusemos até aqui pode ser simetricamente reconstruído para o lado esquerdo. Mais adiante, os conjuntos de denominadores com os quais trabalharemos verificam a condição de Ore bilateralmente. Usaremos, pois, o termo anel (total) de frações para fazer referência a estruturas cujos elementos são descritos em termos tanto da condição L2 ora apresentada, quanto da sua análoga para a esquerda. Encerramos esta discussão com o próximo critério, que fundamenta estas nossas convenções.

Proposição 1.1.10. Se $S$ é um conjunto de Ore à direita e à esquerda e seus elementos são todos regulares em $R$, então $R_{S}$ é, simultaneamente, uma localização à direita e à esquerda de $R$ com respeito a $S$.

Ressaltamos que, nos termos desta última proposição, para todo $x \in R$, devem existir $a_{1}, a_{2} \in R$ e $b_{1}, b_{2} \in S$ tais que $x=a_{1} b_{1}^{-1}=b_{2}^{-1} a_{2}$.

\subsubsection{Anéis de polinômios e extensão de escalares}

De agora em diante, $m$ e $n$ são inteiros positivos, $M_{n}(R)$ é o anel das matrizes $n \times n$ sobre o anel $R,\left\{t_{1}, \ldots, t_{m}\right\}$ é um conjunto de indeterminadas comutativas e $D$ um anel com divisão. O trabalho com matrizes envolve uma porção de índices. Com o fim de não apresentar notações muito carregadas, omitiremos muitas vezes os intervalos nos quais variam tais índices, sempre que o texto trouxer de forma implícita essas informações.

Embora $D$ não seja necessariamente comutativo, muitos resultados que se aplicam aos anéis de polinômios sobre corpos ainda são verdadeiros para $D[t]$. Para os nossos propósitos, enfatizamos que todos os ideais (à esquerda, à direita, bilaterais) de $D[t]$ são principais. Isto é facilmente provado por meio do algoritmo da divisão euclidiana - devemos 
apenas tratar separadamente quocientes e restos à esquerda e quocientes e restos à direita. Uma primeira conseqüência importante deste fato é a possibilidade de estabelecer a noção de máximo divisor comum (à direita, à esquerda) em $D[t]$ : dados $p=p[t]$ e $q=q[t]$ em $D[t]$, basta tomar o gerador mônico do ideal à direita gerado por ambos $p$ e $q$ (e o análogo à esquerda). A segunda conseqüência que podemos extrair desta propriedade do anel de polinômios é o fato de $D[t]$ ser noetheriano, bilateralmente. Como também $D[t]$ é um domínio, usamos a proposição 1.1.9 para concluir que $D[t]$ é um anel de Ore e está embutido em um anel com divisão, a saber, o seu anel total de frações, denotado por $D(t)$. Vamos, agora, elaborar resultados mais abrangentes.

Proposição 1.1.11. O anel de polinômios $D\left[t_{1}, \ldots, t_{m}\right]$ é um anel de Ore, cujo anel total de frações será denotado por $D\left(t_{1}, \ldots, t_{m}\right)$.

Prova: O resultado segue facilmente de uma combinação entre o teorema da base de Hilbert e a proposição 1.1.9. Todavia vamos apresentar uma demonstração direta, por indução sobre $m$. O caso $m=1$ já está validado. Assumamos $m>1$ e que $D\left[t_{1}, \ldots, t_{m-1}\right]$ seja um anel de Ore, com anel total de frações $D\left(t_{1}, \ldots, t_{m-1}\right)$. Portanto $D\left(t_{1}, \ldots, t_{m-1}\right)\left[t_{m}\right]$ é um anel de polinômios na indeterminada comutativa $t_{m}$ com coeficientes em $D\left(t_{1}, \ldots, t_{m-1}\right)$, um anel com divisão. Então podemos formar o anel total de frações $D\left(t_{1}, \ldots, t_{m-1}\right)\left(t_{m}\right)$ e existe um monomorfismo natural $D\left[t_{1}, \ldots, t_{m}\right] \hookrightarrow D\left(t_{1}, \ldots, t_{m-1}\right)\left(t_{m}\right)$ que claramente verifica a condição L1. Resta, portanto, verificar L2. Com efeito, um elemento em $D\left(t_{1}, \ldots, t_{m-1}\right)\left(t_{m}\right)$ é da forma

$$
\frac{\sum_{i}\left(p_{i} q_{i}^{-1}\right) t_{m}^{i}}{\sum_{j}\left(f_{j} g_{j}^{-1}\right) t_{m}^{j}}
$$

para convenientes $p_{i}, q_{i}, f_{j}, g_{j}$ em $D\left[t_{1}, \ldots, t_{m-1}\right]$. Empregamos sucessivas vezes a condição D1 em $D\left[t_{1}, \ldots, t_{m-1}\right]$ e tomamos um denominador comum a todos os $q_{i}$ 's e a todos os $g_{j}$ 's, digamos $0 \neq h$ em $D\left[t_{1}, \ldots, t_{m-1}\right]$. Então a expressão acima é reformulada como segue:

$$
\frac{\sum_{i}\left(p_{i} q_{i}^{-1}\right) t_{m}^{i}}{\sum_{j}\left(f_{j} g_{j}^{-1}\right) t_{m}^{j}}=\frac{\sum_{i}\left(\bar{p}_{i} h^{-1}\right) t_{m}^{i}}{\sum_{j}\left(\bar{f}_{j} h^{-1}\right) t_{m}^{j}}=\frac{\left(\sum_{i} \bar{p}_{i} t_{m}^{i}\right) h^{-1}}{\left(\sum_{j} \bar{f}_{j} t_{m}^{j}\right) h^{-1}}=\frac{\sum_{i} \bar{p}_{i} t_{m}^{i}}{\sum_{j} \bar{f}_{j} t_{m}^{j}} .
$$

Na última expressão à direita, temos que $\sum_{i} \bar{p}_{i} t_{m}^{i}$ e $\sum_{j} \bar{f}_{j} t_{m}^{j}$ estão em $D\left[t_{1}, \ldots, t_{m}\right]$. Isto prova que vale a condição L2 (à direita, mas a prova para o lado esquerdo é análoga) e completa a demonstração.

Usaremos, em argumentos futuros, as relações

$$
D\left(t_{1}, t_{2}\right) \cong D\left(t_{1}\right)\left(t_{2}\right) \cong D\left(t_{2}\right)\left(t_{1}\right)
$$

fundamentadas nos teoremas 1.1.11 e 1.1.6. Para produzir argumentos mais elaborados, introduzimos a próxima

Proposição 1.1.12. Seja $R$ um dominio de Ore, com anel total de frações $R^{\prime}$. Então $M_{n}(R)$ é um anel de Ore, com anel total de frações dado por $M_{n}\left(R^{\prime}\right)$. 
Prova: Inicialmente, observemos que $M_{n}(R)$ é embutido de maneira canônica em $M_{n}\left(R^{\prime}\right)$. Assumamos que uma dada matriz $P \in M_{n}(R)$ seja regular. Para todos $i, j \in\{1, \ldots, n\}$, as equações $\sum_{l=1}^{n} P(i, l) x_{l j}-\delta_{i j} y=0$ nas indeterminadas $x_{i l}, y$ e com coeficientes $R$ formam um sistema linear homogêneo. Temos $n^{2}$ equações - uma para cada par $(i, j)-$ e $n^{2}+1$ indeterminadas - os $x_{l j}$ 's, um para cada par $(l, j)$, e $y$. Como $R$ é embutido em $R^{\prime}$, podemos considerar que os coeficientes estão no anel com divisão $R^{\prime}$. Sucede que o sistema admite uma solução não trivial em $R^{\prime}$, porquanto há mais indeterminadas que equações. Escrevendo uma tal solução com um denominador comum, digamos $x_{l j}=u_{l j} v^{-1}$ e $y=p^{\prime} v^{-1}$, em que os $u_{l j}$ 's, $p^{\prime}$ e $v \neq 0$ estão em $R$, obtemos uma solução não trivial em $R$, a saber $x_{l j}=u_{l j}$ e $y=p^{\prime}$, porque $v$ é uma unidade em $R^{\prime}$ e, para quaisquer $i, j \in\{1, \ldots, n\}$,

$$
0=\sum_{l=1}^{n} P(i, l) u_{l j} v^{-1}-\delta_{i j} p^{\prime} v^{-1}=\left(\sum_{l=1}^{n} P(i, l) u_{l j}-\delta_{i j} p^{\prime}\right) v^{-1} .
$$

Notemos que $p^{\prime} \neq 0$, porque a solução foi tomada não trivial e $P$ é regular. Sejam $U, P^{\prime} \in M_{n}(R)$ dadas por $U(i, j)=u_{i j}$ e $P^{\prime}(i, j)=\delta_{i j} p^{\prime}$. Logo $P U=P^{\prime}$ e a matriz $U^{\prime} \in M_{n}\left(R^{\prime}\right)$ dada por $U^{\prime}(i, j)=\delta_{i j} p^{-1}$ é tal que $P\left(U U^{\prime}\right)=1$. Agora $P\left(U U^{\prime}\right) P=P$ e, como $P$ é regular, concluímos também que $\left(U U^{\prime}\right) P=1$, portanto $P$ é uma unidade em $M_{n}\left(R^{\prime}\right)$ e está estabelecia a condição $\mathbf{L} 1$.

Como $R^{\prime}$ é um anel total de frações de $R$, resulta que, para qualquer $X \in M_{n}\left(R^{\prime}\right)$, após escrever todas as suas entradas com um denominador comum, temos $X(i, j)=a_{i j} b^{-1}$, em que $a_{i j}, b \in R$ e $b \neq 0$. Portanto as matrizes $A, B \in M_{n}(R)$ dadas por $A(i, j)=a_{i j}$ e $B(i, j)=\delta_{i j} b$ verificam a relação $X=A B^{-1}$ em $M_{n}\left(R^{\prime}\right)$, que é a condição L2 para o lado direito. Como esta demonstração pode ser analogamente reconstruída para o lado esquerdo (essencialmente porque $R^{\prime}$ é um anel total de frações bilateralmente de $R$ ), o resultado segue.

Estabeleçamos as seguintes notações: $\mathfrak{R}=M_{n}(D)$ e $\mathfrak{M}_{[t]}=M_{n}(D[t])$. Afirmamos que o anel de polinômios $\mathfrak{R}[t]$ é isomorfo a $\mathfrak{M}_{[t]}$. Inicialmente, tratemos da estrutura aditiva de ambos. Vemos facilmente que $\mathfrak{R}[t]$ é um $\mathfrak{R}$-módulo livre à esquerda, com base $\left\{t^{0}=\right.$ $\left.1, t, t^{2}, \ldots\right\}$. $\mathfrak{M}_{[t]}$ também é um $\mathfrak{R}$-módulo livre à esquerda, com base $\left\{T^{0}=1, T, T^{2}, \ldots\right\}$, em que $T^{k}(i, j)=\delta_{i j} t^{k}$, para todo $k \geq 0$. O $\mathfrak{R}$-homomorfismo de $\mathfrak{R}[t]$ em $\mathfrak{M}_{[t]}$ determinado pela aplicação $t^{k} \mapsto T^{k}, k \geq 0$ é não só aditivo, como também multiplicativo e preserva o elemento identidade. Como é uma bijeção - porquanto leva uma base noutra base -, concluímos que $\mathfrak{R}[t] \cong \mathfrak{M}_{[t]}$ como anéis.

Proposição 1.1.13. $\mathfrak{R}\left[t_{1}, \ldots, t_{m}\right]$ e $\mathfrak{M}_{\left[t_{1}, \ldots, t_{m}\right]}=M_{n}\left(D\left[t_{1}, \ldots, t_{m}\right]\right)$ são isomorfos como anéis.

Prova: A afirmação é provada por indução em $m$. O caso $m=1$ já foi estabelecido acima. Suponhamos $m>1$ e que $\mathfrak{R}\left[t_{1}, \ldots, t_{m-1}\right] \cong \mathfrak{M}_{\left[t_{1}, \ldots, t_{m-1}\right]}$. Então podemos considerar $\mathfrak{R}\left[t_{1}, \ldots, t_{m}\right]$ e $\mathfrak{M}_{\left[t_{1}, \ldots, t_{m}\right]}$ como módulos livres à esquerda sobre $\mathfrak{R}\left[t_{1}, \ldots, t_{m-1}\right]$, aquele com 
base $\left\{t_{m}^{0}=1, t_{m}, t_{m}^{2}, \ldots\right\}$ e este com base $\left\{T_{m}^{0}=1, T_{m}, T_{m}^{2}, \ldots\right\}$, conforme a notação que estabelecemos acima. O restante da demonstração segue exatamente os mesmos passos do caso $m=1$.

Corolário 1.1.14. $\mathfrak{R}\left[t_{1}, \ldots, t_{m}\right]$ é um anel de Ore e seu anel total de frações é dado por $\mathfrak{R}\left(t_{1}, \ldots, t_{m}\right)=\mathfrak{M}_{\left(t_{1}, \ldots, t_{m}\right)}=M_{n}\left(D\left(t_{1}, \ldots, t_{m}\right)\right)$.

Prova: Pela proposição 1.1.12, $\mathfrak{M}_{\left(t_{1}, \ldots, t_{m}\right)}$ é um anel total de frações de $\mathfrak{M}_{\left[t_{1}, \ldots, t_{m}\right]}$. Usamos a proposição 1.1 .13 e o teorema 1.1.6 para afirmar que $\mathfrak{M}_{\left(t_{1}, \ldots, t_{m}\right)}$ também é um anel total de frações de $\mathfrak{R}\left[t_{1}, \ldots, t_{m}\right]$.

Observação 1.1.15. É importante salientar que, na demonstração da proposição 1.1.12, a matriz $P^{\prime}$ obtida por meio da resolução do sistema linear homogêneo é uma matriz escalar não nula, e o conjunto das matrizes escalares é um subanel de $M_{n}(R)$, canonicamente identificado com $R$, donde $P^{\prime}$ é identificada como um elemento de $R$. Combinando esses argumentos com o corolário acima, podemos afirmar que, dado um polinômio regular $p=$ $p[t] \in \mathfrak{R}[t]$, existem $u=u[t], p^{\prime}=p^{\prime}[t] \in \mathfrak{R}[t]$ tais que $p u=p^{\prime}$ e $0 \neq p^{\prime} \in D[t]$, em que $D[t]$ está devidamente identificado como um subanel de $\mathfrak{R}[t]$.

Para referências mais adiante, registramos o seguinte

Corolário 1.1.16. Sejam $t_{1}, t_{2}, \ldots$ indeterminadas comutativas. Então $\mathfrak{R}\left[t_{1}, t_{2}, \ldots\right]$ é um anel de Ore, cujo anel total de frações será denotado por $\mathfrak{R}\left(t_{1}, t_{2}, \ldots\right)$.

Prova: Segue diretamente das demonstrações acima que o anel total de frações do anel de polinômios em infinitas indeterminadas é dado por

$$
\bigcup_{i=1}^{\infty} \mathfrak{R}\left(t_{1}, \ldots, t_{i}\right),
$$

e, em particular, $D\left[t_{1}, t_{2}, \ldots\right]$ é um anel de Ore, cujo anel total de frações será denotado por $D\left(t_{1}, t_{2}, \ldots\right)$.

Já mostramos que $\mathfrak{R}[t]$ é um anel de Ore. Estamos agora interessados em determinar um anel de frações obtido a partir de $\mathfrak{R}[t]$, que, conforme veremos futuramente, é um subanel de $\mathfrak{R}(t)$. Precisamos, então, desenvolver mais o nosso texto. Começamos com a próxima

Proposição 1.1.17. Sejam $F$ um corpo, $R$ uma F-álgebra e $A$ e $B$ duas subálgebras. São equivalentes as seguintes afirmações:

1. O F-homomorfismo $\psi$ de $A \otimes_{F} B$ em $R$ (como espaços vetoriais) induzido pela aplicação $(a, b) \mapsto a b$ de $A \times B$ em $R$ é injetivo

2. Qualquer base de $A$ sobre $F$ é linearmente independente sobre $B$

3. Qualquer base de $B$ sobre $F$ é linearmente independente sobre $A$ 
4. Se $\left\{a_{i}\right\}$ e $\left\{b_{j}\right\}$ são bases de $A$ e de $B$, respectivamente, sobre $F$, então $\left\{a_{i} b_{j}\right\}$ é um conjunto linearmente independente sobre $F$.

Prova: Fixemos uma base $\left\{u_{i}\right\}$ de $A$ sobre $F$. Sejam $v_{1}, \ldots, v_{k}$ em $B$ tais que $\sum_{j} u_{i_{j}} v_{j}=$ 0. Então

$$
0=\sum_{j=1}^{k} u_{i_{j}} v_{j}=\psi\left(\sum_{j=1}^{k} u_{i_{j}} \otimes v_{j}\right)
$$

Se assumirmos 1, concluímos que $\sum u_{i_{j}} \otimes v_{j}=0$ e, com maior razão, $v_{1}=\cdots=v_{k}=0$. Portanto 1 implica 2.

Sejam agora $\left\{a_{i}\right\}$ e $\left\{b_{j}\right\}$ bases de $A$ e de $B$, respectivamente, sobre $F$. Suponhamos que

$$
0=\sum_{i j} \lambda_{i j} a_{i} b_{j}=\sum_{i j} a_{i}\left(\lambda_{i j} b_{j}\right)=\sum_{i} a_{i}\left(\sum_{j} \lambda_{i j} b_{j}\right)
$$

em que, a menos de um número finito, todo $\lambda_{i j} \in F$ é nulo. Se assumirmos $\mathbf{2}$, concluímos imediatamente que, para todo $i, \sum_{j} \lambda_{i j} b_{j}=0$, o que acarreta $\lambda_{i j}$, para quaisquer $i, j$. Então 2 implica 4.

Claramente 4 implica 1 e, de maneira análoga, podemos provar que as implicações acima são verdadeiras se substituirmos 2 por 3 . A demonstração está completa.

Diremos que duas subálgebras nas condições da proposição 1.1 .17 são linearmente disjuntas. Podemos complementar essas idéias com a seguinte

Proposição 1.1.18. Sejam $F$ um corpo, $R$ uma $F$-álgebra e $A$ e $B$ duas subálgebras. Então $R \cong A \otimes_{F} B$ se as três condições abaixo são verificadas:

T1 $a b=b a$, para quaisquer $a \in A$ e $b \in B$.

T2 $A B=R$.

T3 $A$ e $B$ são linearmente disjuntas.

Prova: As condições T1 e T2 mostram que a transformação $F$-linear de $A \otimes_{F} B$ em $R$ induzida pela aplicação $(a, b) \mapsto a b$ é multiplicativa e sobrejetiva. Por T3, tal transformação é, ainda, injetiva, completando esta prova.

Vamos usar estes últimos resultados para sofisticar o nosso entendimento estrutural de alguns dos anéis já apresentados. Denotemos por $K$ o centro do anel com divisão $D$, portanto $K$ é um corpo. Seja $C \subseteq K$ um subcorpo. Pela proposição 1.1.18, pondo $F=C$, $A=\mathfrak{R}, B=C[t]$ e $R=\mathfrak{R}[t]$ (identificando da devida maneira $C[t]$ como um subanel de $\mathfrak{R}[t])$, vemos imediatamente que $\mathfrak{R}[t] \cong \mathfrak{R} \otimes_{C} C[t]$ - em particular, $D \otimes_{C} C[t]$ é o subanel de $\mathfrak{R}[t]$ identificado com $D[t]$-, e, daqui em diante, não faremos distinção entre esses anéis. Usando um argumento simples de indução, provamos também que $\mathfrak{R}\left[t_{1}, \ldots, t_{m}\right] \cong$ $\mathfrak{R} \otimes_{C} C\left[t_{1}, \ldots, t_{m}\right]$. Resulta que, fixando uma base $\left\{V_{j} \mid j \in J\right\}$ de $\mathfrak{R}$ sobre $C$, então um polinômio arbitrário $p[t] \in \mathfrak{R}[t]$ escreve-se unicamente como $p[t]=\sum_{j \in J} V_{j} \otimes p_{j}[t]$, em 
que, a menos de um número finito, todo $p_{j}[t] \in C[t]$ é nulo e, manifestamente, $\left\{V_{j} \otimes t^{i} \mid\right.$ $j \in J, i \geq 0\}$ é uma base de $\mathfrak{R}[t]$ sobre $C$.

Para referências mais adiante, afirmamos que $\mathfrak{R} \otimes_{C} C(t) \hookrightarrow \mathfrak{R}(t)$. Com efeito, a subálgebra $C(t)$ de $\mathfrak{R}(t)$ é central, o que estabelece T1. Resta mostrar que $C(t)$ e $\mathfrak{R}$ são subálgebras linearmente disjuntas de $\mathfrak{R}(t)$. De fato, sejam $r_{1}(t), \ldots, r_{k}(t) \in C(t)$ tais que

$$
\sum_{i=1}^{k} V_{j_{i}} r_{i}(t)=0 .
$$

Após transpor os $r_{i}$ 's a um denominador comum e, após cancelar tal denominador da equação acima, obtemos

$$
\sum_{i=1}^{k} V_{j_{i}} \bar{r}_{i}[t]=0,
$$

em que $\bar{r}_{j}[t] \in C[t]$. Conforme as observações do parágrafo acima, $\mathfrak{R}$ e $C[t]$ são linearmente disjuntas. Então, pela proposição 1.1.17, necessariamente temos $\bar{r}_{i}[t]=0$, para todo $i$. Logo $r_{i}(t)=0$ e, usando mais uma vez 1.1.17, concluímos que a afirmação é verdadeira. Mais uma vez, facilmente concluímos que $\mathfrak{R} \otimes_{C} C\left(t_{1}, \ldots, t_{m}\right) \hookrightarrow \mathfrak{R}\left(t_{1}, \ldots, t_{m}\right)$, usando indução.

Agora, seja $\alpha$ algébrico sobre $C$, portanto o corpo $C(\alpha)$ é uma extensão finita de $C$. Denotemos por $m_{\alpha}=m_{\alpha}[t] \in C[t]$ o polinômio minimal de $\alpha$ sobre $C$, com $\partial m_{\alpha}=r$. Escrevamos $\mathfrak{D}_{\alpha}=\mathfrak{R} \otimes_{C} C(\alpha)$. A aplicação $V_{j} \otimes t^{i} \mapsto V_{j} \otimes \alpha^{i}$ estende-se unicamente a um homomorfismo $\pi_{\alpha}: \mathfrak{R}[t] \rightarrow \mathfrak{D}_{\alpha}$ de $C$-espaços vetoriais. $\pi_{\alpha}$ deve ser sobrejetivo, porquanto $\left\{V_{j} \otimes \alpha^{i} \mid j \in J, 0 \leq i \leq r-1\right\}$ é uma base de $\mathfrak{D}_{\alpha}$ sobre $C$. $\pi_{\alpha}$ é também multiplicativo, porque $t$ é uma indeterminada comutativa, e preserva o elemento identidade, logo é um epimorfismo de $C$-álgebras. Podemos escrever $\pi_{\alpha}(p[t])=p[\alpha]=\sum_{j} V_{j} \otimes p_{j}[\alpha]$, donde $p[\alpha]=0$ se e somente se $p_{j}[\alpha]=0$ para todo $j \in J$, ou seja, se e somente se cada $p_{j}[t]$ pertence a $\left(m_{\alpha}\right)$, o ideal (bilateral) de $C[t]$ gerado por $m_{\alpha}$. Portanto ker $\pi_{\alpha}=\mathfrak{R} \otimes_{C}\left(m_{\alpha}\right)$.

Proposição 1.1.19. $\mathfrak{D}_{\alpha}$ é identificado como um subanel de $M_{r n^{2}}(D)$. Ademais, todo elemento em $\mathfrak{D}_{\alpha}$ que não é divisor de zero à direita é uma unidade em $\mathfrak{D}_{\alpha}$. Em particular, todo elemento em $\mathfrak{D}_{\alpha}$ ou é uma unidade ou é divisor de zero de ambos os lados.

Prova: Observemos que $\mathfrak{D}_{\alpha}$ tem estrutura natural de $D$-espaço vetorial. Seja $\mathcal{L}=$ $\mathcal{L}_{D}\left(\mathfrak{D}_{\alpha}\right)$ o anel de todos os endomorfismos $D$-lineares de $\mathfrak{D}_{\alpha}$. Definimos, para cada $x \in \mathfrak{D}_{\alpha}$, a aplicação $\rho_{x}: \mathfrak{D}_{\alpha} \rightarrow \mathfrak{D}_{\alpha}$ dada por $\rho_{x}(y)=y x$. Resulta que $\rho_{x}$ pertence a $\mathcal{L}$ e a aplicação $x \mapsto \rho_{x}$ define um monomorfismo de anéis de $\mathfrak{D}_{\alpha}$ em $\mathcal{L}^{o p}$, anel oposto de $\mathcal{L}$. Como $\operatorname{dim}_{D} \mathfrak{D}_{\alpha}=r n^{2}$ (o que imediatamente implica $\mathfrak{D}_{\alpha}$ ser um $D$-espaço vetorial artiniano), temos a relação $\mathcal{L}^{o p} \cong M_{r n^{2}}(D)$.

Para demonstrar o que resta, suponhamos que $x$ não seja divisor de zero à direita. Por um lado, obtemos que $\rho_{x}$ é um $D$-endomorfismo injetivo de $\mathfrak{D}_{\alpha}$. Por outro lado, $\mathfrak{D}_{\alpha}$ é artiniano à esquerda, do que decorre $\rho_{x}$ ser um automorfismo de $\mathfrak{D}_{\alpha}$ como $D$-espaço vetorial. Existe, pois, $x^{\prime}$ em $\mathfrak{D}_{\alpha}$ que verifica $1=\rho_{x}\left(x^{\prime}\right)=x^{\prime} x$. Resulta desta relação que 
$x^{\prime}$ não é divisor de zero à direita; repetindo os mesmos argumentos, obtemos $x^{\prime \prime}$ em $\mathfrak{D}_{\alpha}$ que verifica $x^{\prime \prime} x^{\prime}=1$. Necessariamente, $x^{\prime \prime}=x$, o que encerra esta prova.

Proposição 1.1.20. Se $C=K$, Então $\mathfrak{D}_{\alpha}$ é isomorfo a um anel de matrizes sobre um anel com divisão.

Prova: Observemos que os ideais à esquerda de $\mathfrak{D}_{\alpha}$ são invariantes sob a ação de $D$. Conseqüentemente, usando proposição 1.1.19, concluímos que cada ideal à esquerda de $\mathfrak{D}_{\alpha}$ também possui dimensão finita sobre $D$. Então $\mathfrak{D}_{\alpha}$ é um anel artiniano (à esquerda) e, após mostrarmos que é também um anel simples, o resultado seguirá do teorema de Wedderburn-Artin.

Com efeito, seja $I$ um ideal (bilateral) não nulo em $\mathfrak{D}_{\alpha}$. Afirmamos que $I=\mathfrak{D}_{\alpha}$. De fato, tomamos $u=\sum_{i=0}^{r-1} U_{i} \otimes \alpha^{i} \in I$ não nulo de modo que o número de $U_{i}$ 's não nulos na expressão dada - ao que nos referiremos como comprimento de $u$ - seja mínimo. Seja $k \in\{0, \ldots, r-1\}$ o índice do primeiro somando não nulo, então reescrevemos $u=$ $\sum_{i=k}^{r-1} U_{i} \otimes \alpha^{i}$. Como $\mathfrak{R}$ é um anel simples, é verdadeiro que $\mathfrak{R} U_{k} \mathfrak{R}=\mathfrak{R}$, donde obtemos convenientes $A_{j}, B_{j} \in \mathfrak{R}$ tais que $\sum_{j=1}^{s} A_{j} U_{k} B_{j}=1$, para algum $s \geq 1$. Consideremos o seguinte elemento de $I$ :

$$
u^{\prime}=\sum_{j=1}^{s}\left(A_{j} \otimes 1\right) u\left(B_{j} \otimes 1\right) \in I
$$

ou explicitamente

$$
u^{\prime}=1+\sum_{i=k+1}^{r-1} U_{i}^{\prime} \otimes \alpha^{i}, \text { em que } U_{i}^{\prime}=\sum_{j=1}^{s} A_{j} U_{i} B_{j} .
$$

Vemos que o comprimento de $u^{\prime}$ não supera o de $u$, que é mínimo. Redunda que $u^{\prime}$ é um elemento não nulo de $I$ de comprimento também mínimo. Para qualquer $V \in \mathfrak{R}$, temos que

$$
u^{\prime \prime}=(V \otimes 1) u^{\prime}-u^{\prime}(V \otimes 1)=\sum_{i=k+1}^{r-1}\left(V U_{i}^{\prime}-U_{i}^{\prime} V\right) \otimes \alpha^{i}
$$

pertence a $I$ e tem comprimento estritamente inferior ao de $u^{\prime}$. Necessariamente, $u^{\prime \prime}=0$, donde $V U_{i}^{\prime}=U_{i}^{\prime} V$ para todo $i$. Como $V$ é qualquer, concluímos que os $U_{i}$ 's são centrais em $\mathfrak{R}, \operatorname{logo} U_{i}=a_{i} \cdot 1$ com $a_{i} \in K$ para todo $i$. Sucede que $u^{\prime}=1+\sum a_{i} \cdot 1 \otimes \alpha^{i}=$ $1+\sum 1 \otimes a_{i} \alpha^{i} \in 1 \otimes_{K} K(\alpha)$, portanto $u^{\prime}$ é uma unidade e concluímos que $I=\mathfrak{D}_{\alpha}$, como afirmamos.

Para referências mais adiante, temos o

Corolário 1.1.21. Para quaisquer $\alpha_{1}, \ldots, \alpha_{s}$ algébricos sobre $K$, temos que $\mathfrak{D}_{\left(\alpha_{1}, \ldots, \alpha_{s}\right)}=$ $\mathfrak{R} \otimes_{K} K\left(\alpha_{1}, \ldots, \alpha_{s}\right)$ é isomorfo a um anel de matrizes sobre um anel com divisão.

Prova: Já provamos o caso $s=1$. Suponhamos que $\mathfrak{D}_{\left(\alpha_{1}, \ldots, \alpha_{s-1}\right)} \cong M_{p}(\Delta)$, em que $\Delta$ é um anel com divisão. Cômputos simples revelam que $K_{s-1}=K\left(\alpha_{1}, \ldots, \alpha_{s-1}\right)$ é o centro de $\mathfrak{D}_{\left(\alpha_{1}, \ldots, \alpha_{s-1}\right)}$. De acordo com a proposição 1.1.18, pondo $F=K_{s-1}, A=\mathfrak{D}_{\left(\alpha_{1}, \ldots, \alpha_{s-1}\right)}, B=$ 
$K_{s}=K_{s-1}\left(\alpha_{s}\right)$ e $R=\mathfrak{D}_{\left(\alpha_{1}, \ldots, \alpha_{s}\right)}$, temos $\mathfrak{D}_{\left(\alpha_{1}, \ldots, \alpha_{s}\right)} \cong \mathfrak{D}_{\left(\alpha_{1}, \ldots, \alpha_{s-1}\right)} \otimes_{K_{s-1}} K_{s}$ e o resultado segue, como no caso $s=1$, pela proposição 1.1.20.

Seja $S_{\alpha}=\left\{p[t] \in \mathfrak{R}[t] \mid p[\alpha]\right.$ é regular em $\left.\mathfrak{D}_{\alpha}\right\}$, manifestamente um conjunto multiplicativo. Afirmamos que se trata de um conjunto de denominadores em $\mathfrak{D}_{\alpha}$. A princípio, mostremos que seus elementos são todos regulares. Seja $p=p[t] \in \mathfrak{R}[t]$ não nulo e não regular, i.e., um divisor de zero bilateral, de acordo com a proposição 1.1.19. Se $p[\alpha]=0$, então $p \notin S_{\alpha}$. Suponhamos, pois, que $p[\alpha] \neq 0$ e seja $u=u[t] \in \mathfrak{R}[t]$ não nulo tal que $p u=0$, donde $p[\alpha] u[\alpha]=0$. A prova da afirmação estará completa quando mostrarmos que pode ocorrer $u[\alpha] \neq 0$. Relembremos que $\left\{V_{j} \mid j \in J\right\}$ denota uma base de $\mathfrak{R}$ sobre $C$. Podemos escolher $u=u[t]=\sum_{j} V_{j} \otimes u_{j}[t]$ de modo que $\max _{j} \partial u_{j}$ seja o menor possível e esta condição implica $u[\alpha] \neq 0$; caso contrário, $u \in \mathfrak{R} \otimes_{C}\left(m_{\alpha}\right)$, i.e., $u_{j}=u_{j}^{\prime} m_{\alpha}$ com $u_{j}^{\prime}=u_{j}^{\prime}[t] \in C[t]$ para todo $j$. Conseqüentemente, $0=p u=p\left(\sum_{j} V_{j} \otimes u_{j}^{\prime}\right)\left(1 \otimes m_{\alpha}\right)$. Como

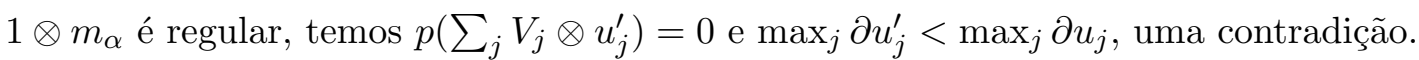

Dado $p=p[t] \in S_{\alpha}$, podemos agora usar a observação 1.1.15 para obter polinômios não nulos $u=u[t], p^{\prime}=p^{\prime}[t] \in \mathfrak{R}[t]$ tais que $p u=p^{\prime}$ e $p^{\prime} \in D \otimes_{C} C[t] \cong D[t]$. Escrevamos $u=\sum_{k} U_{k} \otimes t^{k}$ e denotemos por $E_{i j} \in \mathfrak{R}$ a matriz $i j$-elementar. Então

$$
u=\sum_{i j}\left(E_{i j} \otimes 1\right) u_{i j}
$$

em que

$$
u_{i j}=u_{i j}[t]=\sum_{k}\left(U_{k}(i, j) \cdot 1\right) \otimes t^{k} \in D[t]
$$

Suponhamos que $u$ e $p^{\prime}$ foram escolhidos de modo que $\partial p^{\prime}$ seja o menor possível. Afirmamos que esta propriedade adicional implica $p^{\prime} \in S_{\alpha}$. Pela proposição 1.1.19, é suficiente mostrar que $p^{\prime}[\alpha]$ não é um divisor de zero em $D \otimes_{C} C(\alpha)$. Se $v[t] \in D[t]$ é tal que $p^{\prime}[\alpha] v[\alpha]=0$, temos, por um lado, $p^{\prime} v \equiv 0\left(\bmod \operatorname{ker} \pi_{\alpha}\right)$. Também $p[\alpha] u[\alpha] v[\alpha]=0$, o que acarreta $0=u[\alpha] v[\alpha]=\sum_{i j}\left(E_{i j} \otimes 1\right) u_{i j}[\alpha] v[\alpha]$, porque $p \in S_{\alpha}$. Então $u_{i j} v \equiv 0\left(\bmod \operatorname{ker} \pi_{\alpha}\right)$ para todos $i, j$. Relembremos que os ideais à esquerda em $D[t]$ são principais. Como $\partial p^{\prime}$ é mínimo, não deve haver um divisor comum aos $u_{i j}$ 's e a $p^{\prime}$ com grau positivo, portanto existem $f=f[t], g_{i j}=g_{i j}[t] \in D[t]$ tais que

$$
\sum_{i j} g_{i j} u_{i j}+f p^{\prime}=1
$$

por conseguinte

$$
v=\sum_{i j} g_{i j} u_{i j} v+f p^{\prime} v \equiv 0\left(\bmod \operatorname{ker} \pi_{\alpha}\right),
$$

e, assim, $v[\alpha]=0$, o que prova a afirmação.

Nosso último passo é provar que, dados $q_{1}=q_{1}[t], \ldots, q_{r}=q_{r}[t] \in D[t]$, existem $u_{i}^{\prime}=u_{i}^{\prime}[t], b=b[t] \in D[t]$ tais que $p^{\prime} u_{i}^{\prime}=q_{i} b, 1 \leq i \leq r$, com $b[\alpha]$ regular. Dado $q=q[t] \in D[t]$, existe uma solução não trivial em $D[t]$ para a equação $p^{\prime} x-q y=0$ nas indeterminadas $x$ e $y$, digamos $x=u^{\prime}=u^{\prime}[t]$ e $y=b=b[t]$. Novamente, se escolhemos $u^{\prime}$ e $b$ de modo que $\partial b$ é mínimo, então $b \in S_{\alpha}$. Assumamos que ocorram as relações 
$p^{\prime} u_{i}^{\prime \prime}=q_{i} b_{1}, 1 \leq i \leq r-1$, com $b_{1} \in S_{\alpha}$. Como no caso $r=1$, existem $u_{r}^{\prime}, b_{2} \in D[t]$ tais que $p^{\prime} u_{r}^{\prime}=q_{r} b_{1} b_{2}$ com $b_{2} \in S_{\alpha}$. Denotamos $u_{i}^{\prime}=u_{i}^{\prime \prime} b_{2}, 1 \leq i \leq r-1$, e $b=b_{1} b_{2}$ e o resultado segue.

De acordo com o parágrafo anterior, para qualquer outro polinômio $q=q[t]=$ $\sum_{i j}\left(E_{i j} \otimes 1\right) q_{i j}[t] \in \mathfrak{R}[t]$, com $q_{i j}[t] \in D[t]$, existem $u_{i j}^{\prime}[t], b[t] \in D[t]$ tais que $p^{\prime} u_{i j}^{\prime}=q_{i j} b$ para todos $i, j$ e $b \in S_{\alpha}$. Escrevemos $u^{\prime}[t]=\sum_{i j}\left(E_{i j} \otimes 1\right) u_{i j}^{\prime}[t]$; conseqüentemente $p a=q b$, em que $a=a[t]=u[t] u^{\prime}[t]$. Isto estabelece a condição de Ore à direita para $S_{\alpha}$. A demonstração para o lado esquerdo segue por simetria.

$\mathrm{O}$ anel de frações de $\mathfrak{R}[t]$ com denominadores em $S_{\alpha}$ será denotado por $\mathfrak{R}_{\alpha}(t)$. Como os elementos de $S_{\alpha}$ são regulares, temos $\mathfrak{R}[t] \hookrightarrow \mathfrak{R}_{\alpha}(t)$ e, além disso, o monomorfismo canônico $\iota: \mathfrak{R}[t] \rightarrow \mathfrak{R}(t)$ é $S_{\alpha}$-invertível. Resulta, pelo corolário 1.1.5, que $\mathfrak{R}_{\alpha}(t)$ é subanel de $\mathfrak{R}(t)$. Temos também a propriedade de que todo elemento regular em $\mathfrak{D}_{\alpha}$ é uma unidade, então $\pi_{\alpha}$ também é $S_{\alpha}$-invertível. Sucede, pelo lema 1.1.4, que existe uma única extensão $\epsilon_{\alpha}: \mathfrak{R}_{\alpha}(t) \rightarrow \mathfrak{D}_{\alpha}$ dada por $\epsilon_{\alpha}\left(a b^{-1}\right)=\pi_{\alpha}(a) \pi_{\alpha}(b)^{-1}$, para quaisquer $a=a[t] \in \mathfrak{R}[t] \mathrm{e}$ $b=b[t] \in S_{\alpha}$. Finalmente, observamos que, se $\epsilon_{\alpha}\left(a b^{-1}\right)$ é invertível, então $\pi_{\alpha}(a)$ não pode ser um divisor de zero à direita, o que equivale a dizer que, em conformidade com o a proposição $1.1 .19, \pi_{\alpha}(a)$ é uma unidade em $\mathfrak{D}_{\alpha}$. Redunda que $a \in S_{\alpha}$, logo $a b^{-1}$ é uma unidade em $\mathfrak{R}_{\alpha}(t)$, com inverso $b a^{-1}$. Para encerrar esta seção, sintetizamos os fatos verificados nestes últimos parágrafos no próximo lema, do qual faremos extenso uso nos capítulos que virão.

Lema 1.1.22. 1. $S_{\alpha}=\left\{p[t] \in \mathfrak{R}[t] \mid p[\alpha]=\pi_{\alpha}(p[t])\right.$ é regular em $\left.\mathfrak{D}_{\alpha}\right\}$ é um conjunto de denominadores e $\mathfrak{R}_{\alpha}(t)$, o anel de frações de $\mathfrak{R}[t]$ com denominadores em $S_{\alpha}$, é um subanel de $\mathfrak{R}(t)$.

2. O epimorfismo $\pi_{\alpha}$ admite uma única extensão $\epsilon_{\alpha}: \mathfrak{R}_{\alpha}(t) \rightarrow \mathfrak{D}_{\alpha}$ tal que, para todos $a[t] \in \mathfrak{R}[t]$ e $b[t] \in S_{\alpha}$, temos $\epsilon_{\alpha}\left(a[t] b[t]^{-1}\right)=\pi_{\alpha}(a[t]) \pi_{\alpha}(b[t])^{-1}=a[\alpha] b[\alpha]^{-1}$. Ademais, se $\epsilon_{\alpha}\left(a[t] b[t]^{-1}\right)$ é uma unidade, então a $[t] b[t]^{-1}$ é uma unidade em $\mathfrak{R}_{\alpha}(t)$.

\subsection{Um pouco de topologia}

Esta seção estabelece algumas relações topológicas entre o anel das séries de Laurent em uma indeterminada comutativa $t$ e com coeficientes em um anel com divisão $D$, denotado por $D((t))$, e o anel total de frações $D(t)$. Ambos são anéis com divisão - este já estudado acima, aquele conforme, por exemplo, [Lam01], p.9.

Definimos uma valorização $\|\cdot\|: D((t)) \rightarrow \mathbb{R}_{+}$dada por $\|0\|=0$ e, para todo elemento não nulo $x=\sum_{-\infty}^{\infty} x_{i} t^{i}$, dada por $\|x\|=2^{-N}$, em que $N=\min \left\{i \in \mathbb{Z} \mid x_{i} \neq 0\right\}$. Nestes moldes, uma métrica $d$ em $D((t))$ é dada por $d(x, y)=\|x-y\|$. Não é difícil mostrar que $d$ é uma ultra-métrica, i.e., verifica a desigualdade $d(x, z) \leq \max \{d(x, y), d(y, z)\}$, para todos $x, y, z \in D((t))$, que é estritamente mais forte que a desigualdade triangular.

Com a topologia $\tau$ induzida por $d,(D((t)), \tau)$ é um anel com divisão topológico, i.e., 
as operações de adição e de multiplicação são contínuas e a aplicação de $D((t))-\{0\}$ em si mesmo dada por $x \mapsto x^{-1}$ é também contínua. Destacamos que $(D((t)), \tau)$ verifica o axioma de separação $T_{1}$, i.e., dados dois elementos distintos $x, y \in D((t))$, sempre existe $U_{x} \in \tau$ tal que $x \in U_{x}$ e $y \notin U_{x}$. Isto equivale a dizer que, para qualquer $x \in D((t)),\{x\}$ é um conjunto fechado. O próximo resultado é de nosso interesse.

Proposição 1.2.1. Com a topologia $\tau$ definida acima, $D(t)$ é um subanel denso em $D((t))$.

Prova: Inicialmente, notemos que existe um monomorfismo natural de $D[t]$ em $D((t))$, e que todo elemento regular daquele é uma unidade neste. Resulta, pelo corolário 1.1.5, que $D(t)$ é identificado como um subanel de $D((t))$.

Agora é suficiente mostrar que, para arbitrários $r>0$ e $x \in D((t))$, temos

$$
B_{r}(x) \cap D(t) \neq \emptyset,
$$

em que

$$
B_{r}(x)=\{y \in D((t)) \mid d(x, y)<r\} .
$$

Se $x \in D(t)$, não há o que demonstrar. Caso contrário, necessariamente $x \neq 0$, logo $x=\sum_{n \geq N} x_{n} t^{n}$, para algum $N \in \mathbb{Z}$. Como $\lim _{n \rightarrow \infty} 2^{-n}=0$, existe $N_{0} \geq N$ tal que $2^{-n}<r$, para todo $n \geq N_{0}$. Tomamos $p(t)=\sum_{n=N}^{N_{0}} x_{n} t^{n} \in D(t)$. Pela hipótese sobre $x$, temos $x-p(t) \notin D(t)$, logo existe $N_{1}>N_{0}$ tal que $0 \neq x-p(t)=\sum_{n \geq N_{1}} x_{n} t^{n}$. Resulta que $d(x, p(t))=2^{-N_{1}}<r$, e a demonstração está completa.

\subsection{Filtros e ultra-produtos}

Nesta seção, desenvolveremos um pouco de trabalho sobre filtros. Apesar de serem construções da lógica formal, os filtros são as mais importantes ferramentas utilizadas nas demonstrações dos resultados principais nos próximos capítulos.

No que seguirá, $\Lambda=\{\lambda\}$ é um conjunto não vazio arbitrário e $\mathcal{P}(\Lambda)$ denota a coleção dos subconjuntos de $\Lambda$.

Definição 1.3.1. Um conjunto não vazio $\mathscr{F} \subset \mathcal{P}(\Lambda)$ é dito ser um filtro (em $\Lambda$ ) se verifica os três axiomas abaixo:

F1 $\emptyset \notin \mathscr{F}$.

F2 Para qualquer subcoleção finita $\mathcal{F} \subset \mathscr{F}, \cap \mathcal{F} \in \mathscr{F}$.

F3 Para quaisquer $S, T \in \mathcal{P}(\Lambda)$, se $S \subseteq T$ e $S \in \mathscr{F}$, então $T \in \mathscr{F}$.

Resulta tão somente da definição que $\Lambda \in \mathscr{F}$. Dado $\mathcal{B} \subseteq \mathcal{P}(\Lambda)$, é razoável perguntarse se podemos construir um filtro adicionando a $\mathcal{B}$ os subconjuntos de $\Lambda$ que contêm algum elemento de $\mathcal{B}$. A próxima proposição estabelece as circunstâncias sob as quais isso pode ser obtido. 
Proposição 1.3.2. Seja $\mathcal{B} \subseteq \mathcal{P}(\Lambda)$. Então a coleção de subconjuntos de $\Lambda$ que contêm um elemento $\mathcal{B}$ é um filtro se e somente se $\mathcal{B}$ goza das seguintes propriedades:

B1 $\mathcal{B} \neq \emptyset e \emptyset \notin \mathcal{B}$

B2 Para quaisquer $B_{1}, B_{2} \in \mathcal{B}$, existe $B_{0} \in \mathcal{B}$ tal que $B_{0} \subseteq B_{1} \cap B_{2}$.

Prova: Assumamos que $\mathcal{B}$ possui as propriedades B1 e B2 e seja $\mathscr{F}$ a coleção de subconjuntos de $\Lambda$ que contêm um elemento de $\mathcal{B}$, então o axioma $\mathbf{F} 3$ é imediatamente verificado. Por B1, $\mathscr{F} \neq \emptyset$ e, para todo $S \in \mathscr{F}$, existe $B \in \mathcal{B}$ não vazio tal que $B \subseteq S$, logo o axioma F1 também é verificado. Por B2 (e um argumento simples de indução), concluímos que qualquer interseção de subcoleções finitas de $\mathcal{B}$ contém um elemento de $\mathcal{B}$, o que estabelece o axioma F2. Segue que $\mathscr{F}$ é um filtro, dito gerado por $\mathcal{B}$.

Reciprocamente, se $\mathscr{F}$ é um filtro, então é não vazio e não possui o conjunto vazio entre seus elementos, o que valida B1 para $\mathcal{B}$. A propriedade B2 segue como um caso particular do fato de $\mathscr{F}$ ser fechado para interseções finitas.

Definição 1.3.3. Um conjunto $\mathcal{B} \subseteq \mathcal{P}(\Lambda)$ que goza das propriedades $\boldsymbol{B} \mathbf{1}$ e $\boldsymbol{B} 2$ é dito ser uma base para o filtro gerado por ele.

Observemos que a coleção de todos os filtros em $\Lambda$ é um conjunto naturalmente ordenado pela relação de inclusão. Isto motiva a seguinte

Definição 1.3.4. Um filtro maximal é dito ser um ultra-filtro.

Proposição 1.3.5. Um filtro $\mathscr{F}$ em $\Lambda$ é um ultra-filtro se e somente se, para qualquer $S \subseteq \Lambda$, ou $S \in \mathscr{F}$, ou $\Lambda-S \in \mathscr{F}$.

Prova: Seja $\mathscr{F}$ um filtro com a propriedade do enunciado. Se $\mathscr{F}^{\prime}$ é um outro filtro que contém $\mathscr{F}$, então, para todo $S \in \mathscr{F}^{\prime}$, deve ocorrer $S \in \mathscr{F}$, senão $\Lambda-S \in \mathscr{F} \subseteq \mathscr{F}^{\prime}$ e, pelo axioma F2, $\emptyset=S \cap(\Lambda-S) \in \mathscr{F}^{\prime}$, uma contradição. Logo $\mathscr{F}^{\prime} \subseteq \mathscr{F}$ e $\mathscr{F}$ é maximal.

Reciprocamente, assumamos que $\mathscr{F}$ seja um ultra-filtro. Se $S \subseteq \Lambda$ não pertence a $\mathscr{F}$, temos que $S \cap T_{1}=\emptyset$, para algum $T_{1} \in \mathscr{F} ;$ caso contrário, $\mathcal{B}=\mathscr{F} \cup\{S \cap T \mid T \in \mathscr{F}\}$ seria uma base para um filtro estritamente maior que $\mathscr{F}$, o que não pode ocorrer. Se também $S^{\prime}=\Lambda-S$ não pertence a $\mathscr{F}$, encontramos $T_{2} \in \mathscr{F}$ tal que $S^{\prime} \cap T_{2}=\emptyset$. Então $T_{1} \cap T_{2}=\Lambda \cap\left(T_{1} \cap T_{2}\right)=\left(S \cup S^{\prime}\right) \cap\left(T_{1} \cap T_{2}\right)=\left(S \cap T_{1} \cap T_{2}\right) \cup\left(S^{\prime} \cap T_{1} \cap T_{2}\right)=\emptyset$, uma contradição. Conseqüentemente $\mathscr{F}$ possui a propriedade enunciada.

Observemos que ultra-filtros existem. Vejamos um exemplo construtivo: para qualquer $\lambda_{0} \in \Lambda$, verificamos facilmente que $\mathcal{B}=\left\{\lambda_{0}\right\}$ é base de um filtro, a saber a coleção de todos os subconjuntos de $\Lambda$ que contêm $\lambda_{0}$. Verificamos facilmente também que, neste caso, $\mathcal{B}$ gera um ultra-filtro. Damos agora um exemplo não construtivo, mas de grande utilidade para os nossos propósitos. Sejam $\mathscr{F}$ um filtro arbitrário e $\mathcal{F}$ a coleção (manifestamente não vazia) de todos os filtros maiores ou iguais a $\mathscr{F}$. Segue do lema de Zorn que $\mathcal{F}$ possui um elemento maximal, que deve ser um ultra-filtro que contém $\mathscr{F}$. 
De agora em diante, $\Lambda=\{\lambda\}$ será considerado como um conjunto de índices. Seja $\left\{R_{\lambda} \mid \lambda \in \Lambda\right\}$ uma família de anéis. Identificamos o produto direto completo $\prod_{\lambda \in \Lambda} R_{\lambda}=$ $\prod R_{\lambda}$ como o anel de todas as funções $f: \Lambda \rightarrow \bigcup_{\lambda \in \Lambda} R_{\lambda}$ tais que $f_{\lambda}=f(\lambda) \in R_{\lambda}$, para todo $\lambda \in \Lambda$, munido da adição e da multiplicação ponto a ponto. Quando $R_{\lambda}=R$ para todo $\lambda$, usamos a notação $\prod R_{\lambda}=R^{\Lambda}$.

Seja $\mathscr{F}$ um filtro em $\Lambda$. Para quaisquer $f, g \in \prod R_{\lambda}$, definimos $f \equiv g(\bmod \mathscr{F})$ se e somente se $\left\{\lambda \in \Lambda \mid f_{\lambda}=g_{\lambda}\right\}$ pertence a $\mathscr{F}$. O próximo resultado mostra que podemos conectar filtros a estruturas algébricas.

Proposição 1.3.6. O conjunto $I_{\mathscr{F}}=\left\{f \in \prod R_{\lambda} \mid f \equiv 0(\bmod \mathscr{F})\right\}$ é um ideal em $\prod R_{\lambda}$.

Prova: Como $\Lambda \in \mathscr{F}$, a função identicamente nula, que leva cada $\lambda$ em 0 , pertence a $I_{\mathscr{F}}$. Para arbitrários $f, f^{\prime} \in I_{\mathscr{F}}$ e $g \in \prod R_{\lambda}$, escrevamos $F=\left\{\lambda \in \Lambda \mid f_{\lambda}=0\right\}$, $F^{\prime}=\left\{\lambda \in \Lambda \mid f_{\lambda}^{\prime}=0\right\}$. Sejam $f^{\prime \prime}=f-f^{\prime}, h=f g$ e $h^{\prime}=g f$. Para completar a demonstração, devemos provar que $F^{\prime \prime}=\left\{\lambda \in \Lambda \mid f_{\lambda}^{\prime \prime}=0\right\}, H=\left\{\lambda \in \Lambda \mid h_{\lambda}=0\right\}$ e $H^{\prime}=\left\{\lambda \in \Lambda \mid h_{\lambda}^{\prime}=0\right\}$ estão em $\mathscr{F}$.

Inicialmente, notemos que $F$ e $F^{\prime}$ estão em $\mathscr{F}$, logo por $\mathbf{F} 1$ e por $\mathbf{F} 2$ temos $\emptyset \neq F \cap F^{\prime}$ e é claro que todo $\lambda$ nesta interseção verifica $f_{\lambda}^{\prime \prime}=0$, conseqüentemente $F \cap F^{\prime} \subseteq F^{\prime \prime}$. Resulta que $F^{\prime \prime} \in \mathscr{F}$, por F3. Como a multiplicação é definida ponto a ponto, $F$ está contido em ambos $H$ e $H^{\prime}$. Novamente por F3, segue que $H$ e $H^{\prime}$ estão em $\mathscr{F}$.

Denotamos o anel quociente $\left(\prod R_{\lambda}\right) / I_{\mathscr{F}}$ por $\prod R_{\lambda} / \mathscr{F}$. Particularmente quando $\mathscr{F}$ é um ultra-filtro, $\prod R_{\lambda} / \mathscr{F}$ é dito ser um ultra-produto da família $\left\{R_{\lambda}\right\}$. O próximo resultado é o nosso primeiro exemplo aplicado à álgebra que mostra quão úteis são os ultra-produtos, pela característica que têm de preservar certas propriedades dos anéis subjacentes.

Lema 1.3.7. Seja $\left\{D_{\lambda} \mid \lambda \in \Lambda\right\}$ uma coleção de anéis com divisão e escrevamos $\mathfrak{R}_{\lambda}=$ $M_{n}\left(D_{\lambda}\right)$ para cada $\lambda$. Então, para um ultra-filtro qualquer $\mathscr{F}$ em $\Lambda, \mathcal{D}=\prod D_{\lambda} / \mathscr{F}$ é um anel com divisão e $\prod \mathfrak{R}_{\lambda} / \mathscr{F} \cong M_{n}(\mathcal{D})$.

Prova: Estabeleçamos as seguintes notações:

$$
\begin{aligned}
I_{\mathscr{F}} & =\left\{p \in \prod \mathfrak{R}_{\lambda} \mid p \equiv 0(\bmod \mathscr{F})\right\} \\
J_{\mathscr{F}} & =\left\{f \in \prod D_{\lambda} \mid f \equiv 0(\bmod \mathscr{F})\right\} .
\end{aligned}
$$

Se $f \in \prod D_{\lambda}$ não está em $J_{\mathscr{F}}$, então $F=\left\{\lambda \in \Lambda \mid f_{\lambda}=0\right\} \notin \mathscr{F}$ e segue da proposição 1.3.5 que $F^{\prime}=\Lambda-F \in \mathscr{F}$. Definamos $g \in \prod D_{\lambda}$ dada por $g_{\lambda}=f_{\lambda}^{-1}$ se $\lambda \in F^{\prime}$ e $g_{\lambda}=0$ se $\lambda \in F$ e escrevamos $h=f g$ e $h^{\prime}=g f$. Pela maneira como $g$ foi definida, temos $\left\{\lambda \in \Lambda \mid h_{\lambda}=1\right\}=\left\{\lambda \in \Lambda \mid h_{\lambda}^{\prime}=1\right\}=F^{\prime} \in \mathscr{F}$. Isto mostra que $f+J_{\mathscr{F}} \in \mathcal{D}$ é uma unidade, e a primeira das afirmações é verdadeira.

Para provar a segunda afirmação, para cada $p \in \prod \mathfrak{R}_{\lambda}$, definimos $p_{i j} \in \prod D_{\lambda}$ dada 
por $p_{i j}(\lambda)=p_{\lambda}(i, j)$, isto é, a $i j$-entrada da matriz $p_{\lambda}$. Simples cômputos revelam que

$$
\begin{aligned}
\left(p+p^{\prime}\right)_{i j}(\lambda) & =\left(p_{i j}+p_{i j}^{\prime}\right)(\lambda) \\
\left(p p^{\prime}\right)_{i j}(\lambda) & =\left(\sum_{k=1}^{n} p_{i k} p_{k j}^{\prime}\right)(\lambda)
\end{aligned}
$$

do que sucede

$$
\begin{aligned}
\left(p+p^{\prime}\right)_{i j} & =p_{i j}+p_{i j}^{\prime} \\
\left(p p^{\prime}\right)_{i j} & =\sum_{k=1}^{n} p_{i k} p_{k j}^{\prime} .
\end{aligned}
$$

Como também temos $1_{i j}(\lambda)=\delta_{i j} 1$, concluímos que $\psi: \prod \mathfrak{R}_{\lambda} \rightarrow M_{n}\left(\prod D_{\lambda}\right)$, dado por $\psi(p)=P$, em que $P(i, j)=p_{i j}$, é um homomorfismo de anéis.

Dada arbitrariamente $Q \in M_{n}\left(\prod D_{\lambda}\right)$, definimos $q \in \prod \mathfrak{R}_{\lambda}$ de modo que a $i j$-entrada da matriz $q_{\lambda}$ seja dada por $Q(i, j)(\lambda)$. Resulta imediatamente que $Q=\psi(q)$, $\operatorname{logo} \psi$ é sobrejetivo.

Notemos que $M_{n}\left(J_{\mathscr{F}}\right)$ é um ideal em $M_{n}\left(\prod D_{\lambda}\right)$, e temos $M_{n}\left(\prod D_{\lambda}\right) / M_{n}\left(J_{\mathscr{F}}\right) \cong$ $M_{n}(\mathcal{D})$. Seja $\rho: M_{n}\left(\prod D_{\lambda}\right) \rightarrow M_{n}\left(\prod D_{\lambda}\right) / M_{n}\left(J_{\mathscr{F}}\right)$ a projeção canônica ao quociente. Então $\rho \psi$ é um epimorfismo de anéis. Uma vez provando que $\operatorname{ker}(\rho \psi)=I_{\mathscr{F}}$, o resultado seguirá pelo primeiro teorema do isomorfismo.

Está claro que $p_{\lambda}=0$ se e somente se $0=p_{\lambda}(i, j)=p_{i j}(\lambda)$ para todos $i, j$, donde temos a relação

$$
\left\{\lambda \in \Lambda \mid p_{\lambda}=0\right\}=\bigcap_{i j}\left\{\lambda \in \Lambda \mid p_{i j}(\lambda)=0\right\} .
$$

Podemos combinar os axiomas F2 e F3 para afirmar que $\bigcap_{i j}\left\{\lambda \in \Lambda \mid p_{i j}(\lambda)=0\right\} \in \mathscr{F}$ se e somente se $\left\{\lambda \in \Lambda \mid p_{i j}(\lambda)=0\right\} \in \mathscr{F}$ para todos $i, j$. Concluímos que $p \equiv 0(\bmod \mathscr{F})$ se e somente se $p_{i j} \equiv 0(\bmod \mathscr{F})$ para todos $i, j$, ou seja,

$$
p \in I_{\mathscr{F}} \Longleftrightarrow p_{i j} \in J_{\mathscr{F}}, \forall i, j \in\{1, \ldots, n\}
$$

Como $p \in \operatorname{ker}(\rho \psi)$ se e somente se $P=\psi(p)$ pertence a $M_{n}\left(J_{\mathscr{F}}\right)$, o que equivale a dizer que $p_{i j}=P(i, j) \in J_{\mathscr{F}}$ para quaisquer $i, j$, concluímos que $\operatorname{ker}(\rho \psi)=I_{\mathscr{F}}$.

Relembremos que $\mathfrak{R}=M_{n}(D)$ e que qualquer $p=p[t] \in \mathfrak{R}[t]$ escreve-se unicamente como $p=\sum_{j} V_{j} \otimes p_{j}[t]$, em que $\left\{V_{j} \mid j \in J\right\}$ é uma base para $\mathfrak{R}$ sobre $C$ e $p_{j} \in C[t]$, para todo $j \in J$. Finalizaremos esta seção - e este capítulo - com um resultado bastante importante, que conecta diversos resultados expostos até agora.

Lema 1.3.8. Seja $D$ um anel com divisão de centro infinito $K$. Se $C \subseteq K$ é um subcorpo infinito, então existe um ultra-filtro $\mathscr{F}$ em $C$ tal que $\mathfrak{R}(t)$ é embutido no ultra-produto $\mathfrak{R}^{C} / \mathscr{F}$. 
Prova: No curso desta prova, vamos descrever o seguinte diagrama comutativo:

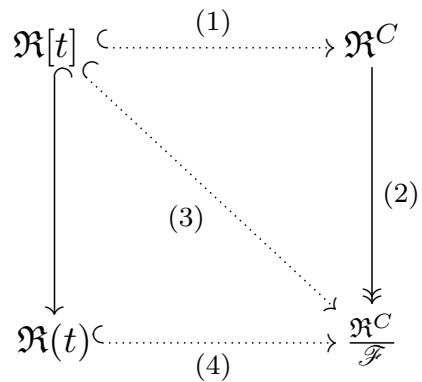

Seja $p=p[t]=\sum_{j} V_{j} \otimes p_{j}[t]$ em $\mathfrak{R}[t]$, com $p_{j} \in C[t]$, para todo $j$. Se $p[c]=$ 0 para todo $c \in C$, então $p_{j}[c]=0$ para todo $j$, porquanto os $V_{j}$ 's são linearmente independentes. Como $C$ é um corpo infinito, temos $p_{j}[c]=0$ para todo $c$ se e somente se $p_{j}=0$. Conseqüentemente, $p[c]=0$ para todo $c \in C$ se e somente se $p=0$. Isso nos permite a estabelecer um monomorfismo (1) de $\mathfrak{R}[t]$ em $\mathfrak{R}^{C}$, dado pela aplicação $p \mapsto f_{p}$, em que $f_{p}(c)=p[c]$.

Para cada $p=p[t] \in \mathfrak{R}[t]$, seja $S_{p}=\{c \in C \mid p[c]$ é regular em $\mathfrak{R}\}$. Verifiquemos que $\mathcal{B}=\left\{S_{p} \mid p[t]\right.$ é regular em $\left.\mathfrak{R}[t]\right\}$ é base de um filtro em $C$. De fato, $\mathcal{B}$ é claramente não vazio e, se $p$ é regular em $\mathfrak{R}[t]$, usamos a observação 1.1.15 para encontrar polinômios $u=u[t]$ e $p^{\prime}=p^{\prime}[t]$ em $\mathfrak{R}[t]$, com $p^{\prime} \in D[t]$ regular, tais que $p u=p^{\prime}$. Como existe ao menos um $c_{0} \in C$ tal que $0 \neq p^{\prime}\left[c_{0}\right] \in D$ (como subanel de $\mathfrak{R}$ ), resulta que $p\left[c_{0}\right] u\left[c_{0}\right]=p^{\prime}\left[c_{0}\right]$, o que implica $p\left[c_{0}\right]$ não ser um divisor de zero à direita. Com maior razão, $p\left[c_{0}\right]$ é regular e, assim, $S_{p} \neq \emptyset$, estabelecendo a condição B1. Dado outro elemento regular $q=q[t] \in \mathfrak{R}[t]$, é claro que $p q$ é regular em $\mathfrak{R}[t]$ e, para um arbitrário $c \in C, p[c] q[c]$ é regular se e somente se $p[c]$ e $q[c]$ são regulares, o que prova $S_{p} \cap S_{q}=S_{p q} \in \mathcal{B}$ e estabelece a condição B2.

Seja $\mathscr{F}$ um ultra-filtro que contém $\mathcal{B}$. Sejam $r=r[t]=\sum_{k} R_{k} \otimes t^{k} \in \mathfrak{R}[t]$ não nulo e $f_{r}$ a sua imagem em $\mathfrak{R}^{C}$. Podemos escrever

$$
r=\sum_{i j}\left(E_{i j} \otimes 1\right) r_{i j}[t]
$$

em que

$$
r_{i j}[t]=\sum_{k}\left(R_{k}(i, j) \cdot 1\right) \otimes t^{k} \in D[t] .
$$

Logo $r[c]=0$ se e somente se $r_{i j}[c]=0$ para todos $i, j$. Como ao menos um dentre os $r_{i j}$ 's é não nulo, digamos, sem perda de generalidade, $0 \neq r_{11}[t] \in D[t]$, então $S_{r_{11}} \in \mathscr{F}$. Por conseguinte, $\{c \in C \mid r[c]=0\} \cap S_{r_{11}}=\emptyset$, o que acarreta

$$
\left\{c \in C \mid f_{r}(c)=0\right\}=\{c \in C \mid r[c]=0\} \notin \mathscr{F} .
$$

Neste caso, $f_{r} \not \equiv 0(\bmod \mathscr{F})$, mostrando que a composição da projeção ao quociente $(2)$ com o monomorfismo (1) é, ainda, um monomorfismo (3).

Finalmente, para qualquer $u=u[t] \in \mathfrak{R}[t]$ regular $-f_{u}$ sua imagem em $\mathfrak{R}^{C}-$, temos $S_{u}=\left\{c \in C \mid f_{u}(c)\right.$ é regular em $\left.\mathfrak{R}\right\} \in \mathscr{F}$ e, para qualquer $c \in S_{u}$, a matriz $f_{u}(c)=u[c]$ é 
regular em $\mathfrak{R}=M_{n}(D)$, portanto uma unidade. Definimos $g \in \mathfrak{R}^{C}$ dada por $g(c)=r[c]^{-1}$ para todo $c \in S_{u}$ e $g(c)=0$, caso contrário. Resulta que $f_{u} g \equiv g f_{u} \equiv 1(\bmod \mathscr{F})$. Usamos o corolário 1.1.5 para estender o monomorfismo (3) a um monomorfismo (4), completando a demonstração 


\section{Capítulo 2}

\section{Identidades racionais}

Este capítulo contém o cerne de todo o texto. Aqui estabeleceremos formalmente o conceito de identidade racional e, reunindo os fatos desenvolvidos no capítulo anterior, produziremos os resultados principais desejados.

\subsection{Identidades Polinomiais}

A fim de motivar o conceito de identidades racionais, podemos fazer um breve recuo na história. Vamos expor um pouco do estudo de uma classe especial de álgebras, as quais satisfazem, em certo sentido, uma propriedade que generaliza a comutatividade. Referimo-nos à classe das álgebras com identidades polinomiais, ou, sucintamente, a classe das PI-álgebras (do inglês, polynomial identity algebras).

As definições e os conceitos elementares no estudo das PI-álgebras envolvem conhecimentos que compõem o repertório de muitos estudantes de álgebra. Partindo deles, podemos produzir resultados sofisticados, como o próximo

Lema 2.1.1 (e.g. Herstein [Her71], p.158). Sejam F um corpo e $n$ um inteiro positivo arbitrários. Então $M_{n}(F)$ não satisfaz uma identidade polinomial de grau inferior a $2 n$.

O próximo resultado é devido a I. Kaplansky. Ele estabelece definitivamente a importância do estudo das PI-álgebras.

Teorema 2.1.2 (Kaplansky [Kap48]). Sejam F um corpo e A uma F-álgebra primitiva. Se A satisfaz uma identidade polinomial, então A é uma álgebra central simples, de dimensão finita sobre o seu centro.

Uma conseqüência imediata do teorema acima é que anéis com divisão de dimensões infinitas sobre seus centros não satisfazem identidades polinomiais. Tendo em vista o lema 2.1.1, o próximo teorema exibe uma identidade polinomial de grau mínimo em um anel de matrizes sobre um corpo. 
Teorema 2.1.3 (Amitsur-Levitzki [AL50]). Sejam $n$ um inteiro arbitrário e $S_{n}$ o grupo simétrico de ordem $n$. Para cada permutação $\sigma$ em $S_{n},(-1)^{\sigma}$ denota o sinal de $\sigma$. Consideremos o polinômio

$$
s_{2 n}=s\left[x_{1}, \ldots, x_{2 n}\right]=\sum_{\sigma \in S_{n}}(-1)^{\sigma} x_{\sigma(1)} \cdots x_{\sigma(2 n)} .
$$

Então, para qualquer corpo $F, s_{2 n}$ é uma identidade polinomial de $M_{n}(F)$.

Destaquemos uma importante aplicação que resulta da combinação dos resultados acima: se $D$ e $E$ são anéis com divisão tais que $D \hookrightarrow E$ e $D$ tem dimensão infinita sobre seu centro, então $E$ também tem dimensão infinita sobre seu centro. Caso contrário, usamos o teorema de Wedderburn-Artin para embutir $E$ em um anel de matrizes sobre um outro anel com divisão e concluímos que $s_{2 n}$ é uma identidade polinomial em $E$, para algum inteiro positivo $n$. Em contrapartida, como as identidades de $E$ são também identidades de $D$, concluímos que $s_{2 n}$ é uma relação polinomial em $D$ e que, a fortiori, $D$ tem dimensão finita sobre seu centro.

Encerramos esta curta seção destacando uma possível extensão (conforme Amitsur [Ami65]) do estudo das identidades polinomiais em uma $F$-álgebra $A$ - que, até então, fazia-se por meio de polinômios na álgebra livre sobre o corpo $F$ em indeterminadas não comutativas do conjunto $\{x\}=\left\{x_{1}, x_{2}, \ldots\right\}$ - a um tipo mais geral de relação polinomial. Os polinômios generalizados são os elementos do produto livre de $A$ e $F\langle x\rangle$ com amálgama em $F$, denotado por $A \underset{F}{*} F\langle x\rangle$, que satisfaz a seguinte propriedade universal: para qualquer $F$-homomorfismo de álgebras $\varphi: A \rightarrow B$ e qualquer função $f:\{x\} \rightarrow B$, existe um único $F$-homomorfismo de álgebras $\Phi: A \underset{F}{*} F\langle x\rangle \rightarrow B$ tal que $\left.\Phi\right|_{A}=\varphi$ e $\left.\Phi\right|_{\{x\}}=f$. Destacamos dois teoremas relacionados a estas observações:

Teorema 2.1.4 (Amitsur [Ami65]). Um anel com divisão satisfaz uma identidade polinomial (generalizada) se e somente se tem dimensão finita sobre seu centro.

Teorema 2.1.5 (Amitsur [Ami65]). Seja D um anel com divisão de centro K. Então $D \underset{K}{*} K[x]$ é um anel sem divisores de zero não triviais que pode ser embutido em um anel com divisão.

O anel com divisão no qual podemos embutir $D \underset{K}{*} K[x]$ é da forma $D^{\Lambda} / \mathscr{F}$, em que $\Lambda$ é a coleção de todos os homomorfismos de $D_{K}^{*} K[x]$ em $D$ e $\mathscr{F}$ é um ultra-filtro conveniente em $\Lambda$, construído em moldes semelhantes aos empregados no lema 1.3.8.

\subsection{Expressões Racionais}

Após o teorema 2.1.5, o último estágio para generalizar identidades polinomiais seria considerar relações que envolvem inversos de um polinômio, algo que, mesmo antes da publicação dos resultados de Amitsur em [Ami66], era feito sob circunstâncias muito 
particulares. Por exemplo, a expressão

$$
a-\left[a^{-1}+\left(b^{-1}-a\right)^{-1}\right]^{-1}-a b a=0,
$$

conhecida como identidade de Hua, é verdadeira para quaisquer $a$ e $b$ em um anel com divisão, desde que $a b \notin\{0,1\}$. Embora possamos classificá-la como uma identidade "trivial", pelo fato de ser uma relação comum a todos os anéis com divisão (sob as devidas restrições), a identidade de Hua pode ser aplicada para obter facilmente o teorema fundamental da geometria projetiva. Uma outra expressão "trivial" que envolve inversos de um polinômio é

$$
(a b)^{-1}-b^{-1} a^{-1}=0,
$$

válida para quaisquer $a$ e $b$ em um anel com divisão, desde que $a, b \notin\{0\}$.

Por ora, as restrições para que as expressões racionais que usamos como exemplo sejam verdadeiras são ainda fáceis de controlar. Para obter resultados gerais, precisamos desenvolver o texto de modo a obter uma formalização efetiva do que chamaremos de identidade racional.

Comecemos com um corpo infinito $C$ e uma $C$-álgebra $A$, na qual elementos invertíveis à esquerda são unidades. Formemos a álgebra livre com unidade $C\langle x\rangle$, em que $\{x\}=\left\{x_{1}, x_{2} \ldots\right\}$ é um conjunto de indeterminadas não comutativas. Reescrevemos (2.1) de uma maneira um pouco mais elaborada:

$$
x_{1}-\frac{1}{\frac{1}{x_{1}}+\frac{1}{\frac{1}{x_{2}}-x_{1}}}-x_{1} x_{2} x_{1} .
$$

A expressão (2.2) ainda não tem sentido formal. Parece ser um elemento de um anel de frações obtido a partir de $C\langle x\rangle$, de cuja existência ainda (e por enquanto) não temos conhecimento. A sequência do texto sugere que a construção de tal anel de frações deve anteceder o estudo das identidades racionais, mas faremos o contrário. Segundo o trabalho de Amitsur [Ami66], a teoria das identidades racionais, cujo ponto de partida é, surpreendentemente, uma álgebra livre sobre $C$ com unidade, antecedeu a sua construção (pioneira, mais uma vez!) de um anel de frações $C(x)$ para a álgebra livre $C\langle x\rangle$. Vejamos, a seguir, uma motivação para introduzir, mas adiante, conceitos formais.

Precisamos aumentar $C\langle x\rangle$ com indeterminadas, digamos, auxiliares. Tomamos $\{y\}=\left\{y_{1}, \ldots, y_{4}\right\}$ de modo que, globalmente, $\{x ; y\}$ é um conjunto de indeterminadas não comutativas. Formamos, então, a álgebra livre com unidade $C\langle x ; y\rangle$. Consideremos os polinômios $p_{1}=p_{1}\langle x\rangle=x_{1}, p_{2}=p_{2}\langle x\rangle=x_{2}, p_{3}=p_{3}\left\langle x ; y_{2}\right\rangle=y_{2}-x_{1}$ e $p_{4}=$ $p_{4}\left\langle x ; y_{1}, y_{3}\right\rangle=y_{1}-y_{3}$. Formamos agora, para cada $j \in\{1, \ldots, 4\}$, o polinômio $q_{j}=$ $q_{j}\left\langle x ; y_{1}, \ldots, y_{j}\right\rangle=y_{j} p_{j}-1$.

Seja $\varphi$ um homomorfismo de $C\langle x ; y\rangle$ em $A$. Se $\varphi\left(q_{j}\right)=0$, para todo $j$, então 
claramente, com as nossas hipóteses,

$$
\begin{aligned}
\varphi\left(x_{1}\right) & =\varphi\left(y_{1}\right)^{-1}, \\
\varphi\left(x_{2}\right) & =\varphi\left(y_{2}\right)^{-1}, \\
\varphi\left(y_{2}\right)-\varphi\left(x_{1}\right)=\varphi\left(y_{2}-x_{1}\right) & =\varphi\left(y_{3}\right)^{-1} \mathrm{e} \\
\varphi\left(y_{1}\right)-\varphi\left(y_{3}\right)=\varphi\left(y_{1}-y_{3}\right) & =\varphi\left(y_{4}\right)^{-1} .
\end{aligned}
$$

Consideremos o polinômio $p=p\langle x ; y\rangle=x_{1}-y_{4}-x_{1} x_{2} x_{1}$ em $C\langle x ; y\rangle$. Usando as relações acima, resulta que

$$
\varphi(p)=\varphi\left(x_{1}\right)-\frac{1}{\frac{1}{\varphi\left(x_{1}\right)}+\frac{1}{\frac{1}{\varphi\left(x_{2}\right)}-\varphi\left(x_{1}\right)}}-\varphi\left(x_{1}\right) \varphi\left(x_{2}\right) \varphi\left(x_{1}\right) .
$$

Comparando o resultado obtido com (2.2), parece razoável investir nesta direção, usando as indeterminadas $y_{j}$ como auxiliares para determinar quais homomorfismos de $C\langle x ; y\rangle$ em $A$ - portanto quais aplicações $x_{i} \mapsto a_{i}$ e $y_{j} \mapsto b_{j}$ - anulam todos os $q_{j}$ 's e fazem o polinômio $p$ acima relacionar-se com a expressão racional em (2.2).

\subsection{Homomorfismos Admissíveis}

Vistas as motivações, introduzimos as definições que seguem. De agora em diante, $C$ denota um corpo infinito e $C\langle x ; y\rangle=C\left\langle x ; y_{1}, \ldots, y_{n}\right\rangle$ é como acima. As $C$-álgebras a serem estudadas satisfazem a propriedade que supusemos anteriormente para $A$ : elementos invertíveis à esquerda são unidades.

Convencionaremos as seguintes notações para subálgebras de $C\langle x ; y\rangle: C_{0}=C\langle x\rangle$ e, para cada $j \in\{1, \ldots, n\}, C_{j}=C\left\langle x ; y_{1}, \ldots, y_{j}\right\rangle$.

Definição 2.3.1. Seja $I_{0}$ um ideal bilateral de $C\langle x ; y\rangle=C\left\langle x ; y_{1}, \ldots, y_{n}\right\rangle$. Diremos que $I_{0}$ é um $Q$-ideal de nivel $\boldsymbol{n}$ (ou simplesmente $Q$-ideal) se $I_{0}=\left(q_{1}, \ldots, q_{n}\right)$ é gerado por polinômios da forma $q_{j}=q_{j}\left\langle x ; y_{1}, \ldots, y_{j}\right\rangle=y_{j} p_{j}-1$, em que $0 \neq p_{j} \in C_{j-1}$, com $1 \leq j \leq n$

Definição 2.3.2. Sejam $R$ uma $C$-álgebra, $\varphi: C\langle x ; y\rangle \rightarrow R$ um $C$-homomorfismo e $I_{0}$ um $Q$-ideal. Então $\varphi$ é dito $\left(I_{0}-\right)$ admissível se $I_{0} \subseteq \operatorname{ker} \varphi$.

Observemos que $\varphi$ é admissível se e somente se $\varphi\left(q_{j}\right)=0$, para todo $j$. A coleção dos homomorfismos admissíveis em $R$ é denotada por $\mathfrak{I}(R)$.

Com as presentes definições, estamos prontos para estabelecer uma relação de sentido entre os polinômios $p=p\langle x ; y\rangle$ em $C\langle x ; y\rangle$ e as expressões racionais, conforme esboçamos na seção anterior.

Definição 2.3.3. Sejam $R$ uma $C$-álgebra e $I_{0}$ um $Q$-ideal. Um polinômio $p=p\langle x ; y\rangle$ é dito uma identidade $\left(I_{0}-\right)$ racional de $R$ se, para todo $\varphi \in \mathfrak{I}(R)$, ocorre $p \in \operatorname{ker} \varphi$. 
Segue da definição acima que, estabelecido um $Q$-ideal, o conjunto das identidades racionais de $R$ é dado por

$$
I(R)=\bigcap_{\varphi \in \mathfrak{I}(R)} \operatorname{ker} \varphi
$$

Observemos que pode ocorrer $\mathfrak{I}(R)=\emptyset$. Neste caso, convencionamos - o que é logicamente plausível $-I(R)=C\langle x ; y\rangle$. Outrossim, uma identidade racional de $R$ que não envolve as indeterminadas $y_{j}$ é uma relação polinomial.

Enfatizamos que, de acordo com as nossas definições, se $\Im(R) \neq \emptyset$ e $\varphi$ é um homomorfismo admissível, então $\varphi(p)=0$ para qualquer identidade polinomial de $R$, ou seja, $p \in I(R)$ e, mesmo quando $\mathfrak{I}(R)=\emptyset$, temos $p \in C\langle x\rangle \hookrightarrow C\langle x ; y\rangle=I(R)$. Isto mostra que o conceito de identidade racional estende o de identidade polinomial.

Nas demonstrações que seguem, está sempre implícito que existe um $Q$-ideal fixado e que as identidades racionais de uma álgebra são aquelas tomadas em relação a esse ideal.

O seguinte lema, de certa forma, é também uma justificativa para o nome identidades racionais.

Lema 2.3.4. Um homomorfismo $\varphi_{0}$ de $C_{0}$ em $R$ origina um homomorfismo admissivel $\varphi=\varphi_{n}: C_{n} \rightarrow R$ se é possível definir, recursivamente, $\varphi_{j}: C_{j} \rightarrow R$ dada por $\left.\varphi_{j}\right|_{C_{j-1}}=$ $\varphi_{j-1}$ e $\varphi_{j}\left(y_{j}\right)=\varphi_{j-1}\left(p_{j}\right)^{-1}$ para cada $j \in\{1, \ldots, n\}$, donde necessariamente $\varphi_{j-1}\left(p_{j}\right)$ é uma unidade em $R$.

Reciprocamente, um homomorfismo admissivel $\varphi: C\langle x ; y\rangle \rightarrow R$ é unicamente determinado por $\varphi_{0}=\left.\varphi\right|_{C_{0}}$.

Prova: A primeira das afirmações é evidente. Para provar a segunda, seja $\varphi: C_{n} \rightarrow R$ um homomorfismos admissível. Denotemos $\varphi_{j}=\left.\varphi\right|_{C_{j}}$. Nestes moldes, $\varphi_{n}=\varphi$. Seja $\tilde{\varphi}: C_{n} \rightarrow R$ um homomorfismo admissível tal que $\tilde{\varphi}_{0}=\varphi_{0}$. Como $p_{1}=p_{1}\langle x\rangle \in C_{0}$, temos que

$$
\tilde{\varphi}\left(y_{1}\right)=\tilde{\varphi}\left(p_{1}\right)^{-1}=\varphi\left(p_{1}\right)^{-1}=\varphi\left(y_{1}\right)
$$

do que decorre $\tilde{\varphi}_{1}=\varphi_{1}$. Assumamos que $\tilde{\varphi}_{n-1}=\varphi_{n-1}$. Como $y_{1}, \ldots, y_{n-1} \in C_{n-1}$, temos $\tilde{\varphi}\left(y_{j}\right)=\varphi\left(y_{j}\right)$, com $1 \leq j \leq n-1$. Além disso, $p_{n}=p_{n}\left\langle x ; y_{1}, \ldots, y_{n-1}\right\rangle \in C_{n-1}$, logo

$$
\tilde{\varphi}\left(y_{n}\right)=\tilde{\varphi}\left(p_{n}\right)^{-1}=\varphi\left(p_{n}\right)^{-1}=\varphi\left(y_{n}\right) .
$$

Como ambos os homomorfismos coincidem nos geradores da álgebra livre $C_{n}$, segue que $\tilde{\varphi}=\varphi$, como queríamos.

Homomorfismos admissíveis são, essencialmente, os $C$-homomorfismos para os quais as imagens dos polinômios $p_{j}$ 's são unidades. O lema acima provê um método de construção de tais homomorfismos bastante útil, além de estabelecer um critério tão simples quanto importante para determinar a unicidade dessas aplicações. Faremos extenso uso das informações nele contidas.

Antes de seguir para o próximo teorema, que envolve fatos esperados sobre identidades racionais, estabeleceremos o seguinte: dada uma família de $C$-álgebras $\left\{R_{\lambda} \mid \lambda \in \Lambda\right\}$, 
definimos, para cada $\lambda_{0} \in \Lambda$, o homomorfismo de projeção $\mu_{\lambda_{0}}: \prod R_{\lambda} \rightarrow R_{\lambda_{0}}$ dado por $\mu_{\lambda_{0}}(f)=f\left(\lambda_{0}\right)$.

Teorema 2.3.5. Sejam $R, R^{\prime}$ e $R_{\lambda}$, para cada $\lambda$ em um conjunto de índices $\Lambda, C$-álgebras arbitrárias. Então são verdadeiras as seguintes afirmações:

1 Se $R \hookrightarrow R^{\prime}$, então $I\left(R^{\prime}\right) \subseteq I(R)$.

$\mathbf{2}$ Se $R \cong R^{\prime}$, então $I(R)=I\left(R^{\prime}\right)$.

$3 I\left(\prod R_{\lambda}\right)=\bigcap_{\lambda \in \Lambda} I\left(R_{\lambda}\right)$.

Prova: Se $\mathfrak{I}(R)=\emptyset$, então $I(R)=C\langle x ; y\rangle$ e o resultado é evidente. Podemos assumir que $\mathfrak{I}(R) \neq \emptyset$, portanto. Sejam $\vartheta: R \hookrightarrow R^{\prime}$ um monomorfismo e $\varphi \in \Im(R)$ arbitrário. Então está bem definido $\vartheta \varphi$ e, $\operatorname{como} \vartheta$ é injetivo, temos $\operatorname{ker} \varphi=\operatorname{ker}(\vartheta \varphi)$, do que decorre $\vartheta \varphi \in \mathfrak{I}\left(R^{\prime}\right)$. É dizer que $\vartheta \Im(R) \subseteq \Im\left(R^{\prime}\right)$. Assim,

$$
I(R)=\bigcap_{\varphi \in \mathfrak{I}(R)} \operatorname{ker} \varphi=\bigcap_{\varphi \in \mathfrak{I}(R)} \operatorname{ker} \vartheta \varphi=\bigcap_{\tilde{\varphi} \in \vartheta \Im(R)} \operatorname{ker} \tilde{\varphi} \supseteq \bigcap_{\tilde{\varphi} \in \mathfrak{I}\left(R^{\prime}\right)} \operatorname{ker} \tilde{\varphi}=I\left(R^{\prime}\right),
$$

o que prova 1 . No caso em que $R \cong R^{\prime}$, digamos que $\psi$ seja um isomorfismo de $R$ em $R^{\prime}$, basta substituir $\vartheta$ acima por $\psi$ e depois por $\psi^{-1}$ para obter a prova de $\mathbf{2}$.

Para mostrar que $\bigcap I\left(R_{\lambda}\right) \subseteq I\left(\prod R_{\lambda}\right)$, podemos, como fizemos acima, supor que $\mathfrak{I}\left(\prod R_{\lambda}\right) \neq \emptyset$. Sejam $p=p\langle x ; y\rangle \in \bigcap I\left(R_{\lambda}\right)$ e $\varphi \in \mathfrak{I}\left(\prod R_{\lambda}\right)$ arbitrários. Então, para cada $\lambda_{0}$ em $\Lambda, \mu_{\lambda_{0}} \varphi$ está em $\mathfrak{I}\left(R_{\lambda_{0}}\right)$, porquanto $I_{0} \subseteq \operatorname{ker} \varphi \subseteq \operatorname{ker}\left(\mu_{\lambda_{0}} \varphi\right)$. Resulta que

$$
0=\left(\mu_{\lambda_{0}} \varphi\right)(p)=(\varphi(p))\left(\lambda_{0}\right)
$$

Como $\lambda_{0}$ é qualquer, concluímos que $\varphi(p)=0$, e a primeira das inclusões está provada. Reciprocamente, para qualquer $\lambda_{0} \in \Lambda$ temos que $\left\{f \in \prod R_{\lambda} \mid f(\lambda)=0, \forall \lambda \neq \lambda_{0}\right\}$ é uma subálgebra de $\prod R_{\lambda}$ isomorfa a $R_{\lambda_{0}}$. Segue, usando $\mathbf{1}$ e $\mathbf{2}$, que também $\bigcap I\left(R_{\lambda}\right) \supseteq I\left(\prod R_{\lambda}\right)$, o que completa a prova da afirmação 3.

Observemos que, como fizemos acima para mostrar inclusões entre conjuntos de identidades racionais, podemos sempre assumir que o conjunto de homomorfismos admissíveis é não vazio. Isto será, a partir de agora, feito nas demais demonstrações.

Teorema 2.3.6. Sejam $R$ uma $C$-álgebra, $\Lambda=\{\lambda\}$ um conjunto de índices, $\left\{R_{\lambda} \mid \lambda \in \Lambda\right\}$ uma família de $C$-álgebras e $\mathscr{F}$ um filtro em $\Lambda$. São verdadeiras as afirmações abaixo:

1 Se $R^{\prime}$ é uma subálgebra de $R^{\Lambda}$, então $I(R) \subseteq I\left(R^{\prime}\right)$. Ademais, se $R_{0}$ é uma subálgebra de $R^{\prime}$ tal que $R_{0} \cong R$, então $I\left(R^{\prime}\right)=I(R)$.

2 Para cada $S \in \mathscr{F}$, definimos $I_{S}=\bigcap_{\lambda \in S} I\left(R_{\lambda}\right)$. Então $I\left(\prod R_{\lambda} / \mathscr{F}\right) \supseteq I_{S}$, para todo $S \in \mathscr{F}$ e, se $\mathscr{F}$ é um ultrafiltro, temos $I\left(\prod R_{\lambda} / \mathscr{F}\right)=\bigcup_{S \in \mathscr{F}} I_{S}$.

$3 I\left(R^{\Lambda} / \mathscr{F}\right)=I(R)$. 
Prova: Suponhamos que $p=p\langle x ; y\rangle \in C\langle x ; y\rangle$ não seja uma identidade racional em $R^{\prime}$. Então existe $\varphi \in \mathfrak{I}\left(R^{\prime}\right)$ tal que $\varphi(p) \neq 0$. Seja $\lambda_{0} \in \Lambda$ tal que $0 \neq(\varphi(p))\left(\lambda_{0}\right)=\left(\mu_{\lambda_{0}} \varphi\right)(p)$. Como ker $\varphi \subseteq \operatorname{ker}\left(\mu_{\lambda_{0}} \varphi\right)$, temos $\mu_{\lambda_{0}} \varphi \in \mathfrak{I}(R)$. Resulta que $p$ não é uma identidade racional em $R$, portanto $I(R) \subseteq I\left(R^{\prime}\right)$. Além disso, se $R_{0}$ é uma subálgebra de $R^{\prime}$ tal que $R_{0} \cong R$, segue do teorema 2.3 .5 que $I\left(R^{\prime}\right) \subseteq I\left(R_{0}\right)=I(R) \subseteq I\left(R^{\prime}\right)$. Logo $I\left(R^{\prime}\right)=I(R)$ e a afirmação 1 é verdadeira.

Para provar a segunda afirmação, fixemos arbitrários $S \in \mathscr{F}, r=r\langle x ; y\rangle \in I_{S}$ e $\varphi \in \mathfrak{I}\left(\prod R_{\lambda} / \mathscr{F}\right)$. Escolhamos $f_{i}, g_{j}$ em $\prod R_{\lambda}$ de modo que $\rho\left(f_{i}\right)=\varphi\left(x_{i}\right)$ e $\rho\left(g_{j}\right)=\varphi\left(y_{j}\right)$, em que $\rho$ é a projeção canônica de $\prod R_{\lambda}$ em $\prod R_{\lambda} / \mathscr{F}$. Seja $\Phi: C\langle x ; y\rangle \rightarrow \prod R_{\lambda}$ o homomorfismo induzido pelas aplicações $x_{i} \mapsto f_{i}$ e $y_{j} \mapsto g_{j}$. Resulta que $\varphi=\rho \Phi$. Como $\varphi$ é admissível, temos $I_{0} \subseteq \operatorname{ker}(\rho \Phi)$. Isto mostra que $\Phi\left(q_{j}\right) \equiv 0(\bmod \mathscr{F})$, para cada gerador $q_{j}$ do $Q$-ideal $I_{0}$, logo

$$
S_{j}=\left\{\lambda \in \Lambda \mid\left(\Phi\left(q_{j}\right)\right)(\lambda)=0\right\}=\left\{\lambda \in \Lambda \mid\left(\mu_{\lambda} \Phi\right)\left(q_{j}\right)=0\right\} \in \mathscr{F}
$$

para cada $j \in\{1, \ldots, n\}$. Seja $T=S \cap\left(\bigcap_{j=1}^{n} S_{j}\right)$. Pelo axioma F2, temos $T \in \mathscr{F}$ e, como $T \subseteq S$, temos também $I_{T} \supseteq I_{S}$, o que acarreta $r \in I_{T}$. Notemos que, para cada $\lambda \in T,\left(\mu_{\lambda} \Phi\right)\left(q_{j}\right)=0$ para todo $j$, ou seja, $\mu_{\lambda} \Phi \in \mathfrak{I}\left(R_{\lambda}\right)$ e, necessariamente, $\left(\mu_{\lambda} \Phi\right)(r)=0$. Nestes moldes,

$$
\left\{\lambda \in \Lambda \mid(\Phi(r))(\lambda)=\left(\mu_{\lambda} \Phi\right)(r)=0\right\} \supseteq T \in \mathscr{F} .
$$

Resulta do axioma F3 que $\{\lambda \in \Lambda \mid(\Phi(r))(\lambda)=0\} \in \mathscr{F}$, portanto $\Phi(r) \equiv 0(\bmod \mathscr{F})$, ou seja, $0=(\rho \Phi)(r)=\varphi(r)$, do que decorre $r \in I\left(\prod R_{\lambda} / \mathscr{F}\right)$. Isto prova a primeira parte da segunda afirmação.

Suponhamos agora que $\mathscr{F}$ seja um ultra-filtro. Pelos argumentos do parágrafo anterior, já sabemos que $\bigcup I_{S} \subseteq I\left(\prod R_{\lambda} / \mathscr{F}\right)$. Para mostrar a inclusão contrária, fixemos $u=u\langle x ; y\rangle \notin \bigcup I_{S}$. Vamos exibir um homomorfismo em $\mathfrak{I}\left(\prod R_{\lambda} / \mathscr{F}\right)$ cujo núcleo não contém $u$. Inicialmente, temos que, para todo $S \in \mathscr{F}$, existem $\lambda_{S} \in S$ e $\psi_{\lambda_{S}} \in \mathfrak{I}\left(R_{\lambda_{S}}\right)$ tais que $\psi_{\lambda_{S}}(u) \neq 0$. Denotemos $T_{u}=\left\{\lambda_{S} \mid S \in \mathscr{F}\right\}$. Desta forma, $T_{u} \cap S \neq \emptyset$ para todo $S \in \mathscr{F}$ e, como este é um ultra-filtro, deve ocorrer $T_{u} \in \mathscr{F}$, conforme os argumentos usados na demonstração da proposição 1.3.5. Sejam $h_{i}, k_{j} \in \prod R_{\lambda}$ dados por $h_{i}\left(\lambda_{S}\right)=\psi_{\lambda_{S}}\left(x_{i}\right)$ e $k_{j}\left(\lambda_{S}\right)=\psi_{\lambda_{S}}\left(y_{j}\right)$ para todo $\lambda_{S} \in T_{u}$, e $h_{i}(\lambda)=0=k_{j}(\lambda)$, para todo $\lambda \in \Lambda-T_{u}$. Seja $\Psi: C\langle x ; y\rangle \rightarrow \prod R_{\lambda}$ o homomorfismo induzido pelas aplicações $x_{i} \mapsto h_{i}$ e $y_{j} \mapsto k_{j}$. Nestes moldes, $\mu_{\lambda_{S}} \Psi$ é um homomorfismo de $C\langle x ; y\rangle$ em $R_{\lambda_{S}}$ que coincide com $\psi_{\lambda_{S}}$ em cada gerador $x_{i}, y_{j}$. Assim, $\mu_{\lambda_{S}} \Psi=\psi_{\lambda_{S}}$, logo $0=\left(\mu_{\lambda_{S}} \Psi\right)\left(q_{j}\right)$, para quaisquer $j \in\{1 \ldots, n\}$ e $\lambda_{S} \in T_{u}$. Além disso, por construção,

$$
\left\{\lambda \in \Lambda \mid\left(\Psi\left(q_{j}\right)\right)(\lambda)=\left(\mu_{\lambda_{S}} \Psi\right)\left(q_{j}\right)=0\right\} \supseteq T_{u} \in \mathscr{F}
$$

o que acarreta, pelo axioma F3, $\left\{\lambda \in \Lambda \mid\left(\Psi\left(q_{j}\right)\right)(\lambda)=0\right\} \in \mathscr{F}$. Logo $(\rho \Psi)\left(q_{j}\right)=0$ para todo $j$, conseqüentemente $\rho \Psi \in \mathfrak{I}\left(\prod R_{\lambda} / \mathscr{F}\right)$. Por outro lado, $(\Psi(u))\left(\lambda_{S}\right)=\psi_{\lambda_{S}}(u) \neq 0$ implica que

$$
\{\lambda \in \Lambda \mid(\Psi(u))(\lambda) \neq 0\} \supseteq T_{u} \in \mathscr{F},
$$


portanto $(\rho \Psi)(u) \neq 0$ e $\rho \Psi$ é o homomorfismo procurado. Isto prova o restante da segunda afirmação.

Para provar a última das afirmações, notemos que, para qualquer $S$ em $\mathscr{F}$, temos, pela afirmação $\mathbf{2}, I(R)=I_{S} \subseteq I\left(R^{\Lambda} / \mathscr{F}\right)$. Reciprocamente, dado $x \in R$, definimos $g_{x} \in R^{\Lambda}$ dado por $g_{x}(\lambda)=x$. Claramente, a aplicação $x \mapsto g_{x}$ é um monomorfismo de anéis e, se $x \neq 0$, temos $g_{x} \not \equiv 0(\bmod \mathscr{F})$. Resulta que $R \hookrightarrow R^{\Lambda} / \mathscr{F}$. Segue do teorema 2.3 .5 que $I(R) \supseteq I\left(R^{\Lambda} / \mathscr{F}\right)$, completando esta demonstração.

\subsection{Extensão de escalares}

Esta seção reúne alguns resultados essenciais para descrever as identidades racionais em álgebras centrais simples de dimensão finita e em álgebras com divisão de dimensão infinita. O nosso objetivo é mostrar que o conjunto das identidades de uma álgebra central simples permanece inalterado se a consideramos (via produto tensorial) como uma $H$-álgebra, em que $H$ é um corpo arbitrário que estende o centro da álgebra central. De agora em diante, $D$ é uma $C$-álgebra com divisão de centro $K$ - portanto $C \hookrightarrow K$ e este é infinito.

Relembremos que $\mathfrak{R}=M_{n}(D)$.

Lema 2.4.1. $I(\mathfrak{R})=I(\mathfrak{R}[t])=I(\mathfrak{R}(t))$. Em particular, $I(D)=I(D[t])=I(D(t))$.

Prova: Temos a sequência de monomorfismos

$$
\mathfrak{R} \hookrightarrow \mathfrak{R}[t] \hookrightarrow \mathfrak{R}(t) \hookrightarrow \mathfrak{R}^{C} / \mathscr{F},
$$

em que o último deles e o ultra-filtro $\mathscr{F}$ estão de acordo com o lema 1.3.8. Combinando os teoremas 2.3.5 e 2.3.6, obtemos

$$
I(\mathfrak{R}) \supseteq I(\mathfrak{R}[t]) \supseteq I(\mathfrak{R}(t)) \supseteq I\left(\mathfrak{R}^{C} / \mathscr{F}\right)=I(\mathfrak{R}),
$$

o que prova a afirmação.

Corolário 2.4.2. $I(\mathfrak{R})=I\left(\Re\left(t_{1}, \ldots, t_{m}\right)\right)$.

Prova: $\mathrm{O}$ caso $m=1$ já foi provado. Suponhamos que $I(\mathfrak{R})=I\left(\mathfrak{R}\left(t_{1}, \ldots, t_{m-1}\right)\right)$. Como vimos, $\mathfrak{R}\left(t_{1}, \ldots, t_{m-1}\right) \cong \mathfrak{M}_{\left(t_{1}, \ldots, t_{m-1}\right)}=M_{n}\left(D\left(t_{1}, \ldots, t_{m-1}\right)\right)$ é uma álgebra de matrizes sobre o anel com divisão $D\left(t_{1}, \ldots, t_{m-1}\right)$, cujo centro é $K\left(t_{1}, \ldots, t_{m-1}\right)$, portanto infinito e contém o corpo $C$. Decorre do lema 2.4.1 que $I\left(\Re\left(t_{1}, \ldots, t_{m-1}\right)\left(t_{m}\right)\right)=$ $I\left(\mathfrak{R}\left(t_{1}, \ldots, t_{m-1}\right)\right)=I(\mathfrak{R})$. Como $\mathfrak{R}\left(t_{1}, \ldots, t_{m-1}\right)\left(t_{m}\right) \cong \mathfrak{R}\left(t_{1}, \ldots, t_{m-1}, t_{m}\right)$, a demonstração está completa.

Para mostrar que as identidades de uma $C$-álgebra não se alteram ao estendermos o corpo de escalares, arrolaremos, a seguir, alguns resultados. O primeiro deles estabelece uma conexão entre os homomorfismos admissíveis em $\mathfrak{R}(t)$ e as construções do lema 1.1.22, e usaremos a notação $\mathfrak{D}_{\alpha}=\mathfrak{R} \otimes_{K} K(\alpha)$, em que $\alpha$ é algébrico sobre $K$. 
Lema 2.4.3. Seja $\varphi: C\langle x ; y\rangle \rightarrow \mathfrak{R}(t)$ um homomorfismo admissivel tal que $\operatorname{Im} \varphi \subseteq \mathfrak{R}_{\alpha}(t)$. Então $\epsilon_{\alpha} \varphi: C\langle x ; y\rangle \rightarrow \mathfrak{D}_{\alpha}$ é admissivel.

Reciprocamente, seja $\psi: C\langle x ; y\rangle \rightarrow \mathfrak{D}_{\alpha}$ um homomorfismo admissível. Então existe $\varphi: C\langle x ; y\rangle \rightarrow \mathfrak{R}_{\alpha}(t)$ admissivel tal que $\epsilon_{\alpha} \varphi=\psi$.

Prova: Como $\operatorname{Im} \varphi \subseteq \mathfrak{R}_{\alpha}(t)$, está bem definido $\epsilon_{\alpha} \varphi$ e $I_{0} \subseteq \operatorname{ker} \varphi \subseteq \operatorname{ker}\left(\epsilon_{\alpha} \varphi\right)$. Segue que $\epsilon_{\alpha} \varphi \in \mathfrak{I}\left(\mathfrak{D}_{\alpha}\right)$.

Para provar a condição recíproca, denotemos $\psi_{j}=\left.\psi\right|_{C_{j}}$, para cada $j \in\{0, \ldots, n\}$. Como $\epsilon_{\alpha}$ é sobrejetivo, podemos escolher, para cada $x_{i}, r_{i}=r_{i}(t) \in \mathfrak{R}_{\alpha}(t)$, de modo que $\epsilon_{\alpha}\left(r_{i}(t)\right)=\psi\left(x_{i}\right)$. Seja $\varphi_{0}$ o homomorfismo de $C_{0}$ em $\mathfrak{D}_{\alpha}$ induzido pela aplicação $x_{i} \mapsto r_{i}$. Resulta imediatamente que $\epsilon_{\alpha} \varphi_{0}=\psi_{0}$. Como $p_{1}=p_{1}\langle x\rangle \in C_{0}$ e $\psi$ é admissível, temos

$$
\left(\epsilon_{\alpha} \varphi_{0}\right)\left(p_{1}\right)=\psi_{0}\left(p_{1}\right)=\psi\left(y_{1}\right)^{-1}
$$

logo $\left(\epsilon_{\alpha} \varphi_{0}\right)\left(p_{1}\right)$ é uma unidade em $\mathfrak{D}_{\alpha}$ e segue do lema 1.1.22 que $\varphi_{0}\left(p_{1}\right)$ é uma unidade em $\mathfrak{R}_{\alpha}(t)$. Definimos $\varphi_{1}: C_{1} \rightarrow \mathfrak{R}_{\alpha}(t)$ dado por $\left.\varphi_{1}\right|_{C_{0}}=\varphi_{0}$ e $\varphi_{1}\left(y_{1}\right)=\varphi_{0}\left(p_{1}\right)^{-1}$. Nestes moldes, é claro que $\left(\epsilon_{\alpha} \varphi_{1}\right)\left(y_{1}\right)=\psi_{1}\left(y_{1}\right)$ e, com maior razão, $\epsilon_{\alpha} \varphi_{1}=\psi_{1}$. Suponhamos que foi definido, para cada $j \in\{2, \ldots, n-1\}$, um homomorfismo $\varphi_{j}: C_{j} \rightarrow \mathfrak{R}_{\alpha}(t)$ de modo que $\left.\varphi_{j}\right|_{C_{j-1}}=\varphi_{j-1}$ e $\epsilon_{\alpha} \varphi_{j}=\psi_{j}$. Como $p_{n}=p_{n}\left\langle x ; y_{1}, \ldots, y_{n-1}\right\rangle \in C_{n-1}$, temos

$$
\left(\epsilon_{\alpha} \varphi_{n-1}\right)\left(p_{n}\right)=\psi_{n-1}\left(p_{n}\right)=\psi\left(y_{n}\right)^{-1}
$$

portanto $\left(\epsilon_{\alpha} \varphi_{n-1}\right)\left(p_{n}\right)$ é uma unidade em $\mathfrak{D}_{\alpha}$. Mais uma vez, pelo lema 1.1.22, segue que $\varphi_{n-1}\left(p_{n}\right)$ é uma unidade em $\mathfrak{R}_{\alpha}(t)$. Estendemos $\varphi_{n-1}$ a $\varphi=\varphi_{n}: C_{n} \rightarrow \mathfrak{R}_{\alpha}(t)$ determinado pelas condições $\left.\varphi\right|_{C_{n-1}}=\varphi_{n-1}$ e $\varphi\left(y_{n}\right)=\varphi_{n-1}\left(p_{n}\right)^{-1}$, o que claramente acarreta $\epsilon_{\alpha} \varphi_{n}=\psi_{n}=\psi$. Segue do lema 2.3.4 que $\varphi=\varphi_{n}$ é um homomorfismo admissível, unicamente determinado por $\varphi_{0}$, com a propriedade enunciada.

Teorema 2.4.4. $I(\mathfrak{R})=I\left(\mathfrak{D}_{\alpha}\right)$.

Prova: Como $\mathfrak{R}$ é uma subálgebra de $\mathfrak{D}_{\alpha}$, segue do teorema 2.3.5 que $I(\mathfrak{R}) \supseteq I\left(\mathfrak{D}_{\alpha}\right)$. Reciprocamente, se $p=p\langle x ; y\rangle \in C\langle x ; y\rangle$ não é uma identidade racional de $\mathfrak{D}_{\alpha}$, existe $\psi \in \mathfrak{I}\left(\mathfrak{D}_{\alpha}\right)$ tal que $\psi(p) \neq 0$. Pelo lema 2.4.3, existe $\varphi \in \mathfrak{I}\left(\mathfrak{R}_{\alpha}(t)\right)$ tal que $\epsilon_{\alpha} \varphi=\psi$. Necessariamente, $\varphi(p) \neq 0$, logo $p$ não é uma identidade racional de $\mathfrak{R}_{\alpha}(t)$. Combinando o lema 1.1.22 e o teorema 2.3.5, concluímos que $I\left(\mathfrak{R}_{\alpha}(t)\right) \supseteq I(\mathfrak{R}(t))$ e, pelo corolário 2.4.1, temos $I(\mathfrak{R}(t))=I(\mathfrak{R})$. Resulta dessas observações que $p \notin I(\mathfrak{R})$. Portanto $I(\mathfrak{R}) \subseteq I\left(\mathfrak{D}_{\alpha}\right)$, o que completa esta demonstração.

Teorema 2.4.5. Para qualquer extensão de corpos $H / K$ finitamente gerada sobre $K$, temos $I(\mathfrak{R})=I\left(\mathfrak{R} \otimes_{K} H\right)$.

Prova: Escrevamos $H=K\left(\xi_{1}, \ldots, \xi_{m}, \alpha_{1}, \ldots, \alpha_{r}\right)$, em que $\left\{\xi_{1}, \ldots, \xi_{m}\right\}$ é uma base de transcendência para $H$ sobre $K$ e $\alpha_{1}, \ldots, \alpha_{r}$ são algébricos sobre $H_{t}=K\left(\xi_{1}, \ldots, \xi_{m}\right)$. 
Então $H_{t} \cong K\left(t_{1}, \ldots, t_{m}\right)$, em que $t_{1}, \ldots, t_{m}$ são indeterminadas comutativas. Temos a sequência de monomorfismos, conforme as discussões do primeiro capítulo,

$$
\mathfrak{R} \hookrightarrow \mathfrak{R} \otimes_{K} K\left(t_{1}, \ldots, t_{m}\right) \hookrightarrow \mathfrak{R}\left(t_{1}, \ldots, t_{m}\right)
$$

portanto segue do teorema 2.3.5 e do corolário 2.4.2 que

$$
I(\mathfrak{R}) \supseteq I\left(\mathfrak{R} \otimes_{K} K\left(t_{1}, \ldots, t_{m}\right)\right) \supseteq I\left(\mathfrak{R}\left(t_{1}, \ldots, t_{m}\right)\right)=I(\mathfrak{R}),
$$

do que resulta $I(\mathfrak{R})=I\left(\mathfrak{R} \otimes_{K} K\left(t_{1}, \ldots, t_{m}\right)\right)=I\left(\mathfrak{R} \otimes_{K} K\left(\xi_{1}, \ldots, \xi_{m}\right)\right)=I\left(\mathfrak{R} \otimes_{K} H_{t}\right)$.

Usamos agora a proposição 1.1.18, considerando as $H_{t}$-álgebras $A=\mathfrak{R} \otimes_{K} H_{t}, B=H$ e $R=\mathfrak{R} \otimes_{K} H$, para obter $\mathfrak{R} \otimes_{K} H \cong\left(\mathfrak{R} \otimes_{K} H_{t}\right) \otimes_{H_{t}} H$. Observemos que

$$
\mathfrak{R} \otimes_{K} H_{t} \cong \mathfrak{R} \otimes_{K} K\left(t_{1}, \ldots, t_{m}\right) \hookrightarrow \mathfrak{R}\left(t_{1}, \ldots, t_{m}\right)
$$

Conseqüentemente,

$$
\mathfrak{R} \hookrightarrow \mathfrak{R} \otimes_{K} H \cong\left(\mathfrak{R} \otimes_{K} H_{t}\right) \otimes_{H_{t}} H \hookrightarrow \mathfrak{R}\left(t_{1}, \ldots, t_{m}\right) \otimes_{H_{t}} H .
$$

Denotemos $\mathfrak{R}^{\prime}=\mathfrak{R}\left(t_{1}, \ldots, t_{m}\right)$. Segue do teorema 2.3.5 e do corolário 2.4.2 que

$$
I(\mathfrak{R}) \supseteq I\left(\mathfrak{R} \otimes_{K} H\right) \supseteq I\left(\mathfrak{R}^{\prime} \otimes_{H_{t}} H\right) .
$$

Nestes moldes, o resultado será estabelecido quando provarmos que $I\left(\mathfrak{R}^{\prime} \otimes_{H_{t}} H\right)=I\left(\mathfrak{R}^{\prime}\right)$, já que, pelo corolário 2.4.2, temos $I\left(\mathfrak{R}^{\prime}\right)=I(\mathfrak{R})$.

Observemos que $\mathfrak{R}^{\prime}$ é isomorfa à álgebra das matrizes $n \times n$ sobre o anel com divisão $D\left(t_{1}, \ldots, t_{m}\right)$, cujo centro é $K\left(t_{1}, \ldots, t_{m}\right) \cong H_{t}$, portanto infinito e também contém $C$. Ademais, $H=H_{t}\left(\alpha_{1}, \ldots, \alpha_{r}\right)$ é uma extensão algébrica e finitamente gerada. Resulta do teorema 2.4.4 que, se $r=1$, então $I\left(\mathfrak{R}^{\prime} \otimes_{H_{t}} H\right)=I\left(\mathfrak{R}^{\prime} \otimes_{H_{t}} H_{t}\left(\alpha_{1}\right)\right)=I\left(\mathfrak{R}^{\prime}\right)$. Se $r>1$, denotamos $H_{r-1}=H_{t}\left(\alpha_{1}, \ldots, \alpha_{r-1}\right)$ e assumimos que $I\left(\mathfrak{R}^{\prime} \otimes_{H_{t}} H_{r-1}\right)=I\left(\mathfrak{R}^{\prime}\right)$. Usando os argumentos do corolário 1.1.21, temos

$$
\mathfrak{R}^{\prime} \otimes_{H_{t}} H \cong\left(\mathfrak{R}^{\prime} \otimes_{H_{t}} H_{r-1}\right) \otimes_{H_{r-1}} H_{r-1}\left(\alpha_{r}\right),
$$

em que $\mathfrak{R}^{\prime} \otimes_{H_{t}} H_{r-1}$ é isomorfo a um anel de matrizes sobre um anel com divisão, cujo centro é $H_{r-1}$. Resulta da relação acima, do teorema 2.4 .4 e da hipótese de indução que $I\left(\mathfrak{R}^{\prime} \otimes_{H_{t}} H\right)=I\left(\mathfrak{R}^{\prime}\right)$, como queríamos.

O seguinte corolário estabelece os propósitos desta seção.

Corolário 2.4.6. Para qualquer extensão de corpos $H / K$, temos $I(\Re)=I\left(\Re \otimes_{K} H\right)$

Prova: Por um lado, temos que $\mathfrak{R} \hookrightarrow \mathfrak{R} \otimes_{K} H$ implica $I(\mathfrak{R}) \supseteq I\left(R \otimes_{K} H\right)$. Suponhamos, agora, que $p=p\langle x ; y\rangle \in C\langle x ; y\rangle$ não seja uma identidade racional de $\mathfrak{R} \otimes_{K} H$. Então existe um homomorfismo admissível $\psi: C\langle x ; y\rangle \rightarrow \mathfrak{R} \otimes_{K} H$ tal que $\psi(p) \neq 0$. Como os polinômios $q_{j}$ (geradores do $Q$-ideal $I_{0}$ ) e $p$ envolvem um número finito de indeterminadas $x_{i}$, deve existir um inteiro positivo $s$ tal que $p, q_{j} \in C_{[s]}=C\left\langle x_{1}, \ldots, x_{s} ; y_{1}, \ldots, y_{n}\right\rangle$, para 
todo $j$. Seja $\varphi: C\langle x ; y\rangle \rightarrow \mathfrak{R} \otimes_{K} H$ o homomorfismo induzido pelas aplicações $x_{i} \mapsto \psi\left(x_{i}\right)$, se $1 \leq i \leq s, x_{i} \mapsto 0$, se $i>s$, e $y_{j} \mapsto \psi\left(y_{j}\right)$, para todo $j$. Por construção, temos $\left.\varphi\right|_{C_{[s]}}=\left.\psi\right|_{C_{[s]}}, \log \varphi(p) \neq 0$ e $\varphi\left(q_{j}\right)=0$, para todo $j$, mostrando que $\varphi \in \mathfrak{I}\left(\Re \otimes_{K} H\right)$.

Pelos argumentos acima, concluímos que a imagem de $\varphi$ está contida em $\mathfrak{R} \otimes_{K} H_{0}$, em que $H_{0}$ é um subcorpo de $H$ finitamente gerado sobre $C$. Em particular, $H_{0}$ é finitamente gerado sobre $K$. Claramente podemos considerar $\varphi \in \mathfrak{I}\left(\Re \otimes_{K} H_{0}\right)$. Como $\varphi(p) \neq 0$ e, pelo teorema 2.4.5, $I\left(\mathfrak{R} \otimes_{K} H_{0}\right)=I(\mathfrak{R})$, segue que $p$ não é uma identidade racional em $\mathfrak{R}$, o que completa esta demonstração.

\section{5 Álgebras centrais simples de dimensão finita}

Pelo teorema de Wedderburn-Artin, uma álgebra central simples de dimensão finita é, a menos de isomorfismo, uma álgebra de matrizes sobre um anel com divisão. A fortiori, a dimensão de uma álgebra central simples sobre seu centro é um quadrado perfeito (e.g. Herstein [Her71], p.92). Usando os resultados da seção anterior, provaremos o seguinte

Teorema 2.5.1. Seja $\mathscr{D}_{n}$ uma álgebra central simples, de centro $K$ tal que $C \subseteq K e$ $\operatorname{dim}_{K} \mathscr{D}_{n}=n^{2}<\infty$. Então $I\left(\mathscr{D}_{n}\right)=I\left(M_{n}(C)\right)=I\left(M_{n}(H)\right)$, para qualquer extensão $H$ de $C$.

Prova: Seja $\bar{K}$ o fecho algébrico de $K$. Então $\operatorname{dim}_{K} \mathscr{D}_{n}=\operatorname{dim}_{\bar{K}}\left(\mathscr{D}_{n} \otimes_{K} \bar{K}\right)$ e $\mathscr{D}_{n} \otimes_{K} \bar{K}$ é, ainda, uma álgebra central simples de centro $\bar{K}$ e, pelo teorema de Wedderburn-Artin, existe um anel com divisão $\Delta$ tal que $\mathscr{D}_{n} \otimes_{K} \bar{K} \cong M_{n}(\Delta)$, do que resulta o centro de $\Delta$ ser isomorfo a $\bar{K}$. Ademais, para qualquer $d \in \Delta,[\bar{K}(d): \bar{K}] \leq n^{2}$, portanto $d$ é algébrico sobre $\bar{K}$, que é algebricamente fechado. Conseqüentemente, $d \in \bar{K}$, o que prova $\Delta \cong \bar{K}$. Como $C \subseteq K \subseteq \bar{K}$ e as matrizes elementares em $M_{n}(C)$ são linearmente independentes sobre $\bar{K}$, concluímos que $M_{n}(\bar{K}) \cong M_{n}(C) \otimes_{C} \bar{K}$. Os mesmos argumentos mostram que, se $H$ é uma extensão de $C$, ainda temos $M_{n}(H) \cong M_{n}(C) \otimes_{C} H$. Usamos o corolário 2.4.6 e o teorema 2.3.5 para concluir

$$
I\left(\mathscr{D}_{n}\right)=I\left(\mathscr{D}_{n} \otimes_{K} \bar{K}\right)=I\left(M_{n}(\bar{K})\right)=I\left(M_{n}(C)\right)=I\left(M_{n}(H)\right),
$$

como queríamos.

Originalmente em [Ami66], teorema 12, Amitsur oferece uma demonstração - baseada em resultados de artigos seus publicados anteriormente - para o fato $I\left(M_{n}(C)\right) \subsetneq$ $I\left(M_{m}(C)\right)$, para quaisquer inteiros positivos $m<n$, uma relação já estabelecida para identidades polinomiais. Em [Ber76a], Bergman apontou, com o consentimento do primeiro autor, um equívoco na prova do referido teorema, retificando-o em seguida: para quaisquer inteiros positivos $m \leq n$, a inclusão $I\left(M_{n}(C)\right) \subseteq I\left(M_{m}(C)\right)$ ocorre se e somente se $m$ divide $n$. Esta condição é suficiente, e podemos usar a teoria que desenvolvemos até aqui para prová-la: se $n=m p$, para algum inteiro positivo $p$, então

$$
M_{m}(C) \hookrightarrow M_{m}(C) \otimes_{C} M_{p}(C) \cong M_{m p}(C)=M_{n}(C)
$$


e a conclusão segue pelo teorema 2.3.5. Essencialmente, restaria mostrar que a condição é também necessária, e isto decorre do citado trabalho de Bergman. Para os nossos propósitos, basta usar a suficiência. Enfatizamos que, se $m$ divide $n$, mas ambos são distintos, então a inclusão entre os conjuntos de identidades racionais é estrita: neste caso, uma combinação do lema 2.1.1 com o teorema 2.1.3 mostra que $s_{2 m}$ está em $I\left(M_{m}(C)\right)$, mas não está em $I\left(M_{n}(C)\right)$. Sintetizamos estas observações no seguinte

Teorema 2.5.2. Para quaisquer inteiros positivos $m \leq n$, então $I\left(M_{n}(C)\right) \subseteq I\left(M_{m}(C)\right)$ se e somente se $m$ divide $n$. Ademais, a igualdade entre os conjuntos de identidades racionais só ocorre se $m=n$.

\section{6 Álgebras com divisão de dimensão infinita}

Esta é a principal seção deste capítulo, na qual descreveremos o conjunto de identidades racionais de uma $C$-álgebra com divisão $D$ de centro $K$, com $\operatorname{dim}_{K} D=\infty$. Os resultados envolvem demonstrações extensas e construções algébricas necessárias para estabelecer o teorema principal (2.6.6).

Nosso primeiro passo é mostrar que as identidades racionais da $C$-álgebra com divisão $D((t))$ (séries de Laurent) são as mesmas de $D$. Isto se inicia com o seguinte

Teorema 2.6.1. Seja $\bar{D}$ um anel com divisão topológico. Se $\bar{D}$ verifica o axioma de separação $T_{1}$ e D é um subanel denso em $\bar{D}$, então $I(\bar{D})=I(D)$.

Prova: Resta mostrar a inclusão $I(\bar{D}) \supseteq I(D)$. Suponhamos que $p=p\langle x ; y\rangle \in C\langle x ; y\rangle$ não seja uma identidade racional de $\bar{D}$. Deve, portanto, existir um homomorfismo admissível $\bar{\varphi}: C\langle x ; y\rangle \rightarrow \bar{D}$ tal que $\bar{\varphi}(p) \neq 0$. Basta, agora, exibir um homomorfismo $\varphi \in \mathfrak{I}(D)$ tal que $\varphi(p) \neq 0$.

Como cada polinômio $q_{j}=y_{j} p_{j}-1$ (gerador do $Q$-ideal $I_{0}$ ) e $p$ envolvem um número finito de indeterminadas $x_{i}$, deve existir um inteiro positivo $s$ tal que $r$ e $q_{j}$ estão em $C_{[s]}=C\left\langle x_{1}, \ldots, x_{s} ; y_{1}, \ldots, y_{n}\right\rangle$, para todo $j$. De acordo com as notações

$$
\bar{\varphi}\left(x_{i}\right)=\bar{d}_{i}, \quad \bar{\varphi}\left(y_{j}\right)=\bar{e}_{j}, \quad \bar{\varphi}\left(p_{j}\right)=\bar{p}_{j} \quad \text { e } \quad \bar{\varphi}(p)=\bar{p},
$$

verificamos que $\bar{e}_{j} \neq 0 \neq \bar{p}_{j}=p_{j}\langle\bar{d} ; \bar{e}\rangle$, para todo $j$, porque $\bar{\varphi}$ é admissível, e $0 \neq \bar{p}=$ $p\langle\bar{d} ; \bar{e}\rangle$, por hipótese.

Seja $d \in \bar{D}$ não nulo. Como $\bar{D}$ verifica $T_{1}$, o conjunto $\{0\}$ é fechado, ou seja, $\bar{D}^{*}$ é um conjunto aberto. Conseqüentemente, se $U(d)$ é uma vizinhança de $d$ em $\bar{D}$, então $U(d) \cap \bar{D}^{*} \subseteq \bar{D}^{*}$ ainda é uma vizinhança de $d$. Reciprocamente, se $U^{\prime}(d)$ é uma vizinhança de $d$ em $\bar{D}^{*}$, então $U^{\prime}(d)$ é da forma $U^{\prime \prime}(d) \cap \bar{D}^{*}$, para alguma vizinhança $U^{\prime \prime}(d)$ de $d$ em $\bar{D}$. Portanto $U^{\prime}(d)$ é também uma vizinhança de $d$ em $\bar{D}$. Além disso, como a adição e a multiplicação são contínuas, para qualquer $f\langle x ; y\rangle \in C_{[s]}$, a aplicação $\left(d_{1}, \ldots, d_{s}, e_{1}, \ldots, e_{n}\right) \mapsto f\langle d ; e\rangle$ de $\bar{D}^{s+n}$ em $\bar{D}$ é contínua. 
Restrinjamos as idéias do parágrafo acima ao nosso caso particular. Fixamos uma vizinhança $U(\bar{p}) \subseteq \bar{D}^{*}$. Então existem vizinhanças $V_{n}\left(\bar{d}_{i}\right)$ e $V_{n}\left(\bar{e}_{j}\right) \subseteq \bar{D}^{*}$, para todos $i, j$, tais que, para quaisquer $d_{i} \in V_{n}\left(\bar{d}_{i}\right)$ e $e_{j} \in V_{n}\left(\bar{e}_{j}\right)$, temos $p\langle d ; e\rangle \in U(\bar{p})$.

Fixemos a vizinhança $V_{n}\left(\bar{e}_{n}\right)$ obtida acima. Observemos que a aplicação $d \mapsto d^{-1}$ de $\bar{D}^{*}$ em si mesmo é contínua e bijetiva, coincidindo com a sua própria inversa; é, pois, um homeomorfismo. Em particular, deve ser uma aplicação aberta, do que resulta $V_{n}\left(\bar{e}_{n}\right)^{-1}=$ $\left\{e_{n}^{-1} \mid e_{n} \in V_{n}\left(\bar{e}_{n}\right)\right\}$ ser uma vizinhança de $\bar{e}_{n}^{-1}=\bar{p}_{n}$ em $\bar{D}^{*}$, logo também em $\bar{D}$. Nestes moldes, deve haver $V_{n-1}\left(\bar{d}_{i}\right)$ e $V_{n-1}\left(\bar{e}_{j}\right) \subseteq \bar{D}^{*}$, para cada $j \in\{1, \ldots, n-1\}$, tais que, para arbitrários $d_{i} \in V_{n-1}\left(\bar{d}_{i}\right), e_{j} \in V_{n-1}\left(\bar{e}_{j}\right)$, com $1 \leq j \leq n-1$,

$$
p_{n}\langle d ; e\rangle=p_{n}\left\langle d ; e_{1}, \ldots, e_{n-1}\right\rangle \in V_{n}\left(\bar{e}_{n}\right)^{-1} .
$$

Por argumentos análogos, temos que $V_{n-1}\left(\bar{e}_{n-1}\right)^{-1}$ é uma vizinhança de $\bar{e}_{n-1}^{-1}=\bar{p}_{n-1}$ em $\bar{D}$. Logo existem $V_{n-2}\left(\bar{d}_{i}\right)$ e $V_{n-2}\left(\bar{e}_{j}\right)$, para cada $j \in\{1, \ldots, n-2\}$ tais que, dados arbitrários $d_{i} \in V_{n-2}\left(\bar{d}_{i}\right)$ e $e_{j} \in V_{n-2}\left(\bar{e}_{j}\right)$, com $1 \leq j \leq n-2$,

$$
p_{n-1}\langle d ; e\rangle=p_{n-1}\left\langle d ; e_{1}, \ldots, e_{n-2}\right\rangle \in V_{n-1}\left(\bar{e}_{n-1}\right)^{-1} .
$$

Este processo deve sobrestar em um número finito de passos, quando obtivermos vizinhanças $V_{j}\left(\bar{d}_{i}\right)$ e $V_{k}\left(\bar{e}_{j}\right)$, com $1 \leq j \leq n$ e $j \leq k \leq n$, nos moldes acima. Definimos, para cada $i \in\{1, \ldots, s\}$,

$$
V\left(\bar{d}_{i}\right)=\bigcap_{j=1}^{n} V_{j}\left(\bar{d}_{i}\right) .
$$

Também definimos, para cada $j \in\{1, \ldots, n\}$,

$$
V\left(\bar{e}_{j}\right)=\bigcap_{k=j}^{n} V_{k}\left(\bar{e}_{j}\right) \subseteq \bar{D}^{*} .
$$

Por serem interseções finitas, $V\left(\bar{d}_{i}\right)$ e $V\left(\bar{e}_{j}\right)$ são vizinhanças de $\bar{d}_{i}$ e de $\bar{e}_{j}$, respectivamente, para todos $i, j$. Outrossim, pela construção acima, dados arbitrários $d_{i} \in V\left(\bar{d}_{i}\right)$ e $e_{j} \in V\left(\bar{e}_{j}\right)$, para todos $i, j$, ainda temos

$$
p_{j}\langle d ; e\rangle=p_{j}\left\langle d ; e_{1}, \ldots, e_{j-1}\right\rangle \in V\left(\bar{e}_{j}\right)^{-1}
$$

e também

$$
p\langle d ; e\rangle \in U(\bar{p}) \subseteq \bar{D}^{*},
$$

porque $V\left(\bar{d}_{i}\right) \subseteq V_{n}\left(\bar{d}_{i}\right)$ e $V\left(\bar{e}_{j}\right) \subseteq V_{n}\left(\bar{e}_{j}\right)$, para todos $i, j$.

Como $D$ é denso em $\bar{D}$, podemos escolher, para cada $i \in\{1, \ldots, s\}, d_{i} \in V\left(\bar{d}_{i}\right) \cap D$. Então $p_{1}\langle d\rangle \in V\left(\bar{e}_{1}\right)^{-1}$, ou seja, existe $e_{1} \in V\left(\bar{e}_{1}\right)$ tal que $e_{1}^{-1}=p_{1}\langle d\rangle \in D$. Determinemos, sucessivamente, os demais elementos $e_{j} \in V\left(\bar{e}_{j}\right)$ tais que $e_{j}^{-1}=p_{j}\left\langle d ; e_{1}, \ldots, e_{j-1}\right\rangle \in$ $D, \operatorname{com} 2 \leq j \leq n$. Resulta que o homomorfismo $\varphi: C\langle x ; y\rangle \rightarrow D$ induzido pelas aplicações $x_{i} \mapsto d_{i}$, se $1 \leq i \leq s, x_{i} \mapsto 0$, se $i>s$ e $y_{j} \mapsto e_{j}$ é admissível e $\varphi(p) \neq 0$, o que completa a demonstração. 
Corolário 2.6.2. Para uma C-álgebra com divisão $D$ qualquer, $I(D)=I(D(t))=$ $I[D((t))]$.

Prova: A demonstração da afirmação segue pela combinação do teorema $2.6 .1 \mathrm{com}$ o lema 2.4.1 e a proposição 1.2.1.

O próximo lema desenvolve uma importante conseqüência do teorema 2.1.5 no contexto das identidades racionais.

Lema 2.6.3. Sejam $D$ uma $C$-álgebra com divisão de centro $K$ e $\{z\}=\left\{z_{1}, z_{2}, \ldots\right\}$ um conjunto de indeterminadas não comutativas. Consideremos o produto livre $\mathcal{R}=D_{K}^{*}\langle z\rangle$ com amálgama em $K$. Então $I(D)=I(\mathcal{R})$.

Prova: Como $D \hookrightarrow \mathcal{R}$ e este é identificado como um subanel de um ultra-produto de cópias de $D$, o qual denotaremos por $\mathcal{Q}$, segue, dos teoremas 2.3.5 e 2.3.6,

$$
I(D) \supseteq I(\mathcal{R}) \supseteq I(\mathcal{Q})=I(D),
$$

o que prova a afirmação.

O próximo lema é muito interessante, por ser o primeiro avanço que fazemos para mostrar que as identidades racionais de uma $C$-álgebra com divisão de dimensão infinita sobre seu centro são triviais. Nele, reuniremos grande parte das construções feitas até agora.

Lema 2.6.4. Seja $D$ uma $C$-álgebra com divisão de centro $K$. Se D é uma subálgebra de uma $C$-álgebra com divisão $E$ e $\operatorname{dim}_{K} D=\infty$, então $I(E)=I(D)$.

Prova: Inicialmente, observemos que, de acordo com as observações feitas na seção 2.1, $E$ deve ser uma $C$-álgebra de dimensão infinita sobre seu centro. O restante da demonstração será tratado em seis passos.

Passo 1: Consideremos o anel $\mathcal{R}[[t]]$ das séries de potência formais em uma indeterminada comutativa $t$ e com coeficientes em $\mathcal{R}=D_{K}^{*} K\langle z\rangle$. Temos, então, a sequência de monomorfismos

$$
\mathcal{R} \hookrightarrow \mathcal{R}[[t]] \hookrightarrow \mathcal{Q}[[t]] \hookrightarrow \mathcal{Q}((t))
$$

do que decorre

$$
I(\mathcal{R}) \supseteq I \mathcal{R}[[t]] \supseteq I(\mathcal{Q}[[t]]) \supseteq I[\mathcal{Q}((t))] .
$$

Pelo lema 2.6.3, temos $I(\mathcal{R})=I(\mathcal{Q})=I(D)$ e, pelo corolário 2.6.2, $I(\mathcal{Q})=I[\mathcal{Q}((t))]$. Logo

$$
I(D)=I(\mathcal{R}[[t]])=I(\mathcal{Q}[[t]]) .
$$

Passo 2: : As unidades do anel $\mathcal{R}[[t]]$ são, precisamente, os elementos $r=\sum_{i=0}^{\infty} r_{i} t^{i}$ tais que $r_{0}$ é uma unidade em $\mathcal{R}$. Seja $\mu_{0}: \mathcal{R}[[t]] \rightarrow \mathcal{R}$ o homomorfismo dado por $\mu_{0}(r)=$ $\mu_{0}\left(\sum_{i=0}^{\infty} r_{i} t^{i}\right)=r_{0}$. Então $r$ é uma unidade em $\mathcal{R}[[t]]$ se e somente se $\mu_{0}(r)$ é uma unidade em $\mathcal{R}$. Nestes termos, é claro que, se $r$ é uma unidade em $\mathcal{R}[[t]]$, temos $\mu_{0}\left(r^{-1}\right)=$ $\mu_{0}(r)^{-1}=r_{0}^{-1}$. 
Passo 3: Fixemos um $Q$-ideal $I_{0}$. Seja $\tau: C\langle x ; y\rangle \rightarrow D$ um homomorfismo admissível arbitrário. Sejam $u$ uma indeterminada comutativa e $D_{u}=D(u)$ o anel total de frações de $D[u]$. Nos moldes do lema 1.1.22, fazendo $\alpha=1$, consideremos o anel de frações $D_{1}(u) \subseteq D_{u}$, cujos denominadores são os elementos de

$$
S_{1}=\left\{p[u] \in D[u] \mid \pi_{1}(p[u]) \text { é regular em } D\right\}=\{p[u] \in D[u] \mid p[1] \neq 0\} .
$$

De acordo com os resultados do primeiro capítulo, temos

$$
D[u] \hookrightarrow D_{1}(u) \hookrightarrow D_{u},
$$

portanto o homomorfismo $\psi_{0}$ induzido pela aplicação $x_{i} \mapsto \tau\left(x_{i}\right) u$ de $C_{0}$ em $D[u]$ pode ser considerado como um homomorfismo de $C_{0}$ em $D_{1}(u)$. Está definido, então, $\epsilon_{1}^{u} \psi_{0}$, em que $\epsilon_{1}^{u}$ é o homomorfismo descrito no lema 1.1.22, com $u \mapsto 1$. Manifestamente $\epsilon_{1}^{u} \psi_{0}=\tau_{0}$. Como $p_{1}=p_{1}\langle x\rangle \in C_{0}$, temos

$$
\left(\epsilon_{1}^{u} \psi_{0}\right)\left(p_{1}\right)=\tau_{0}\left(p_{1}\right)=\tau\left(p_{1}\right) \in \mathcal{U}(D)=D^{*}
$$

Pelo segundo item do lema 1.1.22, segue que $\psi_{0}\left(p_{1}\right) \in \mathcal{U}\left(D_{1}(u)\right)$. Estendemos $\psi_{0}$ ao homomorfismo $\psi_{1}$ determinado pelas condições $\left.\psi_{1}\right|_{C_{0}}=\psi_{0}$ e $\psi_{1}\left(y_{1}\right)=\psi_{0}\left(p_{1}\right)^{-1}$. Claramente, $\epsilon_{1}^{u} \psi_{1}=\tau_{1}$. Suponhamos que foram definidos homomorfismos $\psi_{j}: C_{j} \rightarrow D_{1}(u)$ de modo a verificar $\left.\psi_{j}\right|_{C_{j-1}}=\psi_{j-1}, \psi_{j}\left(y_{j}\right)=\psi_{j-1}\left(p_{j}\right)^{-1}$ e $\epsilon_{1}^{u} \psi_{j}=\tau_{j}$, com $2 \leq j \leq n-1$. Então $\left(\epsilon_{1}^{u} \psi_{n-1}\right)\left(p_{n}\right)=\tau_{n-1}\left(p_{n}\right) \in \mathcal{U}(D)$ e, pelo lema 1.1.22, obtemos $\psi_{n-1}\left(p_{n}\right) \in \mathcal{U}\left(D_{1}(u)\right)$. Estendemos, finalmente, $\psi_{n-1}$ ao homomorfismo $\psi=\psi_{n}$ de $C_{n}=C\langle x ; y\rangle$ em $D_{1}(u)$ determinado pelas condições $\left.\psi\right|_{C_{n-1}}=\psi_{n-1}$ e $\psi\left(y_{n}\right)=\psi_{n-1}\left(p_{n}\right)^{-1}$.

Segue do lema 2.3.4 que $\psi$ é um homomorfismo admissível e, pelo lema 2.4.3, $\epsilon_{1}^{u} \psi$ é, também, admissível. Como $\left.\left(\epsilon_{1}^{u} \psi\right)\right|_{C_{0}}=\epsilon_{1}^{u} \psi_{0}=\tau_{0}$, usamos mais uma vez o lema 2.3.4 para concluir que $\epsilon_{1}^{u} \psi=\tau$.

Passo 4: Sejam $\psi: C\langle x ; y\rangle \rightarrow D_{1}(u)$ o homomorfismo obtido no passo 3 e $D_{u}=$ $D(u)$ como acima, cujo centro é $K_{u}=K(u)$. Denotemos $\mathcal{R}_{u}=D_{u} \underset{K_{u}}{*} K_{u}\langle z\rangle$. Vamos construir um homomorfismo $\varphi: C\langle x ; y\rangle \rightarrow \mathcal{R}_{u}[[t]]$ que verifica $\mu_{0} \varphi=\psi$.

Inicialmente, observemos que existem monomorfismos naturais

$$
D_{1}(u) \hookrightarrow D_{u} \hookrightarrow \mathcal{R}_{u} \hookrightarrow \mathcal{R}_{u}[[t]]
$$

logo $D_{1}(u)$ pode ser identificado como um subanel de $\mathcal{R}_{u}[[t]]$. Seja $\varphi_{0}$ o homomorfismo de $C_{0}$ em $\mathcal{R}_{u}[[t]]$ determinado pela aplicação $x_{i} \mapsto \psi\left(x_{i}\right)+z_{i} t$. Decorre que, para todo $i$,

$$
\left(\mu_{0} \varphi_{0}\right)\left(x_{i}\right)=\psi\left(x_{i}\right)
$$

o que acarreta $\mu_{0} \varphi_{0}=\psi_{0}$. Em particular, concluímos que $\left(\mu_{0} \varphi_{0}\right)\left(C_{0}\right) \subseteq D_{1}(u)$. Como $p_{1}=p_{1}\langle x\rangle \in C_{0}$, temos

$$
\left(\mu_{0} \varphi_{0}\right)\left(p_{1}\right)=\psi_{0}\left(p_{1}\right) \in \mathcal{U}\left(D_{1}(u)\right) \subseteq \mathcal{U}\left(\mathcal{R}_{u}\right)
$$


e segue do passo 2 que $\varphi_{0}\left(p_{1}\right)$ é uma unidade em $\mathcal{R}_{u}[[t]]$. Estendemos $\varphi_{0}$ ao homomorfismo $\varphi_{1}$ de $C_{1}$ em $\mathcal{R}_{u}[[t]]$ determinado pelas condições $\left.\varphi_{1}\right|_{C_{0}}=\varphi_{0}$ e $\varphi_{1}\left(y_{1}\right)=\varphi_{0}\left(p_{1}\right)^{-1}$. Suponhamos que foram definidos homomorfismos $\varphi_{j}: C_{j} \rightarrow \mathcal{R}_{u}[[t]]$ de modo a verificar $\left.\varphi_{j}\right|_{C_{j-1}}=\varphi_{j-1}, \varphi_{j}\left(y_{j}\right)=\varphi_{j-1}\left(p_{j}\right)^{-1}$ e $\mu_{0} \varphi_{j}=\psi_{j}$, com $2 \leq j \leq n-1$. Em particular, $\left(\mu_{0} \varphi_{n-1}\right)\left(p_{n}\right)=\psi_{n-1}\left(p_{n}\right) \in \mathcal{U}\left(\mathcal{R}_{u}\right)$ e, mais uma vez, $\varphi_{n-1}\left(p_{n}\right)$ é uma unidade em $\mathcal{R}_{u}[[t]]$. Estendemos, finalmente, $\varphi_{n-1}$ ao homomorfismo $\varphi_{n}=\varphi$ de $C_{n}$ em $\mathcal{R}_{u}[[t]]$ determinado pelas condições $\left.\varphi\right|_{C_{n-1}}=\varphi_{n-1}$ e $\varphi\left(y_{n}\right)=\varphi_{n-1}\left(p_{n}\right)^{-1}$. Resulta, pelo lema 2.3.4, que $\varphi$ é admissível. Claramente, $\mu_{0} \varphi$ é um homomorfismo admissível de $C_{n}$ em $\mathcal{R}_{u}[[t]]$ tal que $\left.\left(\mu_{0} \varphi\right)\right|_{C_{0}}=\psi_{0}$. Usando mais uma vez o lema 2.3.4, concluímos que $\mu_{0} \varphi=\psi$.

Passo 5: Seja $\lambda: C\langle x ; y\rangle \rightarrow E$ um homomorfismo admissível. Analogamente ao que foi feito no passo 3, existe um homomorfismo admissível $\bar{\sigma}: C\langle x ; y\rangle \rightarrow E_{1}(t)$ que verifica as propriedades $\bar{\sigma}\left(x_{i}\right)=\lambda\left(x_{i}\right) t$ e $\epsilon_{1}^{t} \bar{\sigma}=\lambda$. Conforme os argumentos do primeiro capítulo, existem monomorfismos naturais

$$
D_{u} \hookrightarrow E_{u} \hookrightarrow E_{u}((t)) \quad \text { e } \quad E \hookrightarrow E_{1}(t) \hookrightarrow E(t) \hookrightarrow E_{u}(t) \hookrightarrow E_{u}((t))
$$

A inclusão de $D_{u}$ em $E_{u}$ e a aplicação $z_{i} \mapsto \lambda\left(x_{i}\right)$ de $\{z\}$ em $E_{u}$ induzem um único homomorfismo $\eta_{0}: \mathcal{R}_{u} \rightarrow E_{u}$ que verifica $\eta_{0}\left(z_{i}\right)=\lambda\left(x_{i}\right)$ e cuja restrição a $D_{u}$ é a inclusão deste em $E_{u}$. A fortiori, temos um homomorfismo $\eta: \mathcal{R}_{u}[[t]] \rightarrow E_{u}((t))$, dado por

$$
\eta\left(\sum_{i=0}^{\infty} r_{i} t^{i}\right)=\sum_{i=0}^{\infty} \eta_{0}\left(r_{i}\right) t^{i} .
$$

Sejam $\tau, \psi$ e $\varphi$ como descritos nos passos anteriores. Então $\eta \varphi$ é um homomorfismo admissível que verifica, para todo $i$,

$$
(\eta \varphi)\left(x_{i}\right)=\eta\left(\psi\left(x_{i}\right)+z_{i} t\right)=\eta\left(\tau\left(x_{i}\right) u+z_{i} t\right)=\tau\left(x_{i}\right) u+\lambda\left(x_{i}\right) t .
$$

Considerando os monomorfismos

$$
D \hookrightarrow E_{u} \hookrightarrow E_{u}[t] \hookrightarrow E_{u}(t) \hookrightarrow E_{u}((t))
$$

e os cômputos acima, temos que $(\eta \varphi)\left(C_{0}\right) \subseteq E_{u}(t)=E(u, t)=E_{t}(u)$. Suponhamos $(\rho \varphi)\left(C_{n-1}\right) \subseteq E_{t}(u)$. Então

$$
(\rho \varphi)\left(y_{n}\right)=(\rho \varphi)\left(p_{n}\right)^{-1} \in(\rho \varphi)\left(C_{n-1}\right) \subseteq E_{t}(u),
$$

do que decorre $(\rho \varphi)(C\langle x ; y\rangle) \subseteq E_{t}(u)$.

Passo 6: Este último passo reúne as informações dos anteriores e completa a demonstração, que será feita por indução sobre o nível do $Q$-ideal $I_{0}$, para mostrar que $I(D) \subseteq I(E)$, já que a inclusão contrária é verdadeira, pelo teorema 2.3.5.

Se $n=0$, então $I(D) \subseteq C\langle x\rangle$. Como a dimensão de $D$ sobre $K$ é infinita, usamos o teorema 2.1.2 para concluir que $I(D)=(0) \subseteq I(E)$, estabelecendo, assim, o resultado.

Fixemos um $Q$-ideal $I_{0}=\left(q_{1}, \ldots, q_{n}\right)$, com $q_{j}=y_{j} p_{j}-1$. Suponhamos $n>0$ e que as identidades racionais de $D$ relativas a qualquer $Q$-ideal de nível menor que $n$ sejam as 
mesmas de $E$. Suponhamos que $p=p\langle x ; y\rangle \in C\langle x ; y\rangle$ não seja uma identidade racional de $E$. Então existe um homomorfismo admissível $\lambda: C\langle x ; y\rangle \rightarrow E$ tal que $\lambda(p) \neq 0$. Observemos que

$$
\lambda\left(p_{n}\right)=\lambda\left(p_{n}\left\langle x ; y_{1}, \ldots, y_{n-1}\right\rangle\right)=\lambda\left(y_{n}\right)^{-1},
$$

portanto $p_{n} \notin I_{n-1}(E)$, em que este último é o conjunto das identidades racionais de $E$ na álgebra $C\left\langle x ; y_{1}, \ldots, y_{n-1}\right\rangle$ respectivas ao $Q$-ideal grado pelos polinômios $q_{1}, \ldots, q_{n-1}$. Por hipótese de indução, temos $I_{n-1}(E)=I_{n-1}(D)$, conseqüentemente $p_{n} \notin I_{n-1}(D)$. A princípio, deve haver um homomorfismo admissível $\tau_{0}: C_{n-1} \rightarrow D$ tal que $\tau_{0}\left(p_{n}\right) \neq 0$. Estendemos $\tau_{0}$ ao homomorfismo $\tau: C_{n} \rightarrow D$ determinado por $\left.\tau\right|_{C_{n-1}}=\tau_{0}$ e $\tau\left(y_{n}\right)=$ $\tau_{0}\left(p_{n}\right)^{-1}$. Pelo lema 2.3.4, $\tau$ é $I_{0}$-admissível. Suponhamos que o passo 3 tenha sido refeito para este $\tau$ em particular, derivando a partir daí os demais passos. Obtemos, de acordo com tudo o que construímos, os homomorfismos que se ajustam ao diagrama abaixo, em que $\sigma$ e $\epsilon_{0}^{u}$ ainda serão determinados:

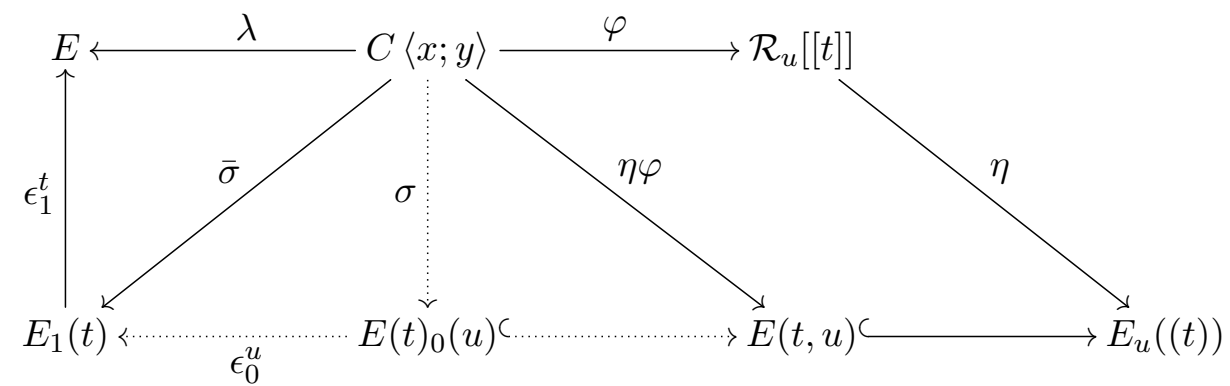

Nos moldes do lema 1.1.22, fazendo $\alpha=0$, obtemos o anel de frações $E(t)_{0}(u)$ a partir de $E_{t}[u]$ e com denominadores em

$$
S_{0}=\left\{p^{t}[u] \in E_{t}[u] \mid \pi_{0}\left(p^{t}[u]\right) \text { é regular em } E_{t}\right\}=\left\{p^{t}[u] \in E_{t}[u] \mid p^{t}[0] \neq 0\right\} .
$$

De acordo com os resultados do primeiro capítulo, temos

$$
E_{t}[u] \hookrightarrow E(t)_{0}(u) \hookrightarrow E_{t}(u)=E(t, u) .
$$

Vamos construir $\sigma: C\langle x ; y\rangle \rightarrow E(t)_{0}(u)$, de modo a verificar as propriedades $\sigma\left(x_{i}\right)=$ $\lambda\left(x_{i}\right) t+\tau\left(x_{i}\right) u$ e $\epsilon_{0}^{u} \sigma=\bar{\sigma}$. Isto é feito de modo inteiramente análogo ao passo 3.

Começamos com o homomorfismo $\sigma_{0}$ de $C_{0}$ em $E(t)_{0}(u)$ determinado pela aplicação $x_{i} \mapsto \lambda\left(x_{i}\right) t+\tau\left(x_{i}\right) u$, do que imediatamente resulta $\epsilon_{0}^{u} \sigma_{0}=\bar{\sigma}_{0}$ e, assim,

$$
\left(\epsilon_{0}^{u} \sigma_{0}\right)\left(p_{1}\right)=\bar{\sigma}_{0}\left(p_{1}\right) \in \mathcal{U}\left(E_{1}(t)\right) .
$$

Conseqüentemente, $\sigma_{0}\left(p_{1}\right)$ é uma unidade em $E(t)_{0}(u)$ e podemos estender $\sigma_{0}$ ao homomorfismo $\sigma_{1}$ de $C_{1}$ em $E(t)_{0}(u)$ determinado pelas condições $\left.\sigma_{1}\right|_{C_{0}}=\sigma_{0}$ e $\sigma_{1}\left(y_{1}\right)=\sigma_{0}\left(p_{1}\right)^{-1}$, do que facilmente decorre $\epsilon_{0}^{u} \sigma_{1}=\bar{\sigma}_{1}$. Suponhamos que tenha sido determinado, para cada $j \in\{2, \ldots, n-1\}$, um homomorfismo $\sigma_{j}$ de $C_{j}$ em $E(t)_{0}(u)$, de modo a verificar $\left.\sigma_{j}\right|_{C_{j-1}}=\sigma_{j-1}, \sigma_{j}\left(y_{j}\right)=\sigma_{j-1}\left(p_{j}\right)^{-1}$ e $\epsilon_{0}^{u} \sigma_{j}=\bar{\sigma}_{j}$. Logo

$$
\left(\epsilon_{0}^{u} \sigma_{n-1}\right)\left(p_{n}\right)=\bar{\sigma}_{n-1}\left(p_{n}\right) \in \mathcal{U}\left(E_{1}(t)\right)
$$


e sucede que $\sigma_{n-1}\left(p_{n}\right)$ é uma unidade em $E(t)_{0}(u)$. Obtemos, finalmente, o homomorfismo $\sigma_{n}=\sigma$, determinado pelas condições $\left.\sigma\right|_{C_{n-1}}=\sigma_{n-1}$ e $\sigma\left(y_{n}\right)=\sigma_{n-1}\left(p_{n}\right)^{-1}$. Segue que $\sigma$ é um homomorfismo admissível tal que

$$
\left.\left(\epsilon_{0}^{u} \sigma\right)\right|_{C_{0}}=\epsilon_{0}^{u} \sigma_{0}=\bar{\sigma}_{0},
$$

o que prova a relação $\epsilon_{0}^{u} \sigma=\bar{\sigma}$. Além disso, como

$$
\sigma_{0}\left(x_{i}\right)=\lambda\left(x_{i}\right) t+\tau\left(x_{i}\right) u=(\eta \varphi)\left(x_{i}\right),
$$

necessariamente $\sigma_{0}=\left.(\eta \varphi)\right|_{C_{0}}$. Com maior razão, $\sigma=\eta \varphi$. Podemos, portanto, resumir o diagrama acima no seguinte diagrama comutativo

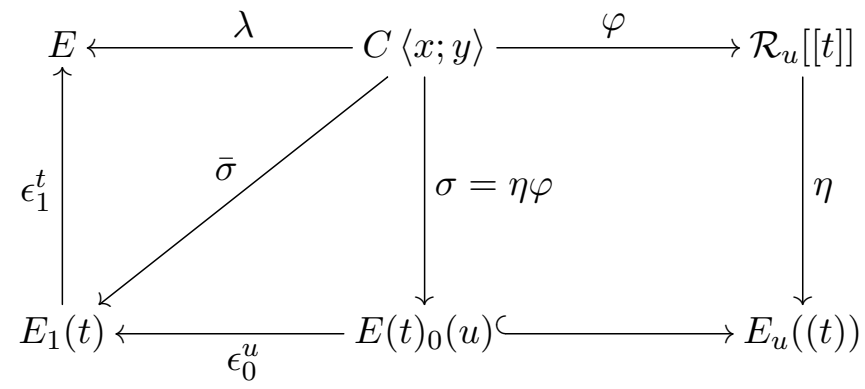

Sucede que

$$
\lambda=\epsilon_{1}^{t} \bar{\sigma}=\epsilon_{1}^{t} \epsilon_{0}^{u} \sigma=\epsilon_{1}^{t} \epsilon_{0}^{u} \eta \varphi,
$$

e concluímos que, como $\lambda(p) \neq 0$, necessariamente $\varphi(p) \neq 0$. Resulta, com auxílio do passo 1 e do lema 2.4.1, que

$$
p \notin I\left(\mathcal{R}_{u}\right)=I\left(D_{u}\right)=I(D),
$$

e a demonstração está completa.

O lema 2.6.4 ainda é um resultado que pressupõe uma relação de inclusão entre as $C$-álgebras. Para provar o resultado principal, precisamos ainda estabelecer uma espécie de ponte entre duas $C$-álgebras com divisão de dimensão infinita arbitrárias. Isto ficará mais claro na sequência do texto. O próximo resultado será fundamental a esse propósito.

Lema 2.6.5. Seja $D$ uma $C$-álgebra com divisão de centro $K$. Então, para cada inteiro positivo $m$, existem:

1 uma extensão $H_{m}$ de $K$;

2 uma $H_{m}$-álgebra central com divisão $\mathscr{D}_{m}$ tal que $\operatorname{dim}_{H_{m}} \mathscr{D}_{m}=m^{2} e$

3 uma $H_{m}$-álgebra com divisão $D_{m}$ que contém ambas $D$ e $\mathscr{D}_{m}$.

Prova: Fixemos um inteiro positivo $m$. Sejam $\xi_{1}, \xi_{2}, \ldots$ e $\eta$ indeterminadas comutativas sobre $K$. Denotemos $H=K(\xi ; \eta)=K\left(\xi_{1}, \xi_{2}, \ldots ; \eta\right)$, portanto uma extensão transcendental de $K$, e $D_{H}=D(\xi ; \eta)=D\left(\xi_{1}, \xi_{2}, \ldots ; \eta\right)$, o total de frações obtido a partir 
de $D[\xi ; \eta]=D\left[\xi_{1}, \xi_{2}, \ldots ; \eta\right]$, conforme o corolário 1.1.16. Como $D$-espaço vetorial, este último anel tem uma base dada por

$$
\left\{v_{J}=\eta^{j_{0}} \xi_{1}^{j_{1}} \cdots \xi_{i}^{j_{i}} \cdots \mid J=\left(j_{0}, j_{1}, \ldots, j_{i}, \ldots\right) \in \bigoplus_{i=0}^{\infty} \mathbb{N}\right\},
$$

que possui a propriedade $v_{J_{1}} \cdot v_{J_{2}}=v_{\left(J_{1}+J_{2}\right)}$. Consideremos a aplicação

$$
\begin{aligned}
\left(^{\prime}\right): \bigoplus_{i=0}^{\infty} \mathbb{N} & \longrightarrow \bigoplus_{i=0}^{\infty} \mathbb{N} \\
J & \longmapsto J^{\prime}=\left(j_{0}, j_{m}, j_{1}, j_{2}, \ldots, j_{m-1}, j_{m+1}, \ldots\right),
\end{aligned}
$$

que verifica a propriedade $\left(J_{1}+J_{2}\right)^{\prime}=J_{1}^{\prime}+J_{2}^{\prime}$.

Existe um único operador $D$-linear $\vartheta_{0}$ de $D[\xi ; \eta]$ tal que $\vartheta_{0}\left(v_{J}\right)=v_{J^{\prime}}$. Claramente, $\vartheta_{0}^{m}=1$, do que resulta $\vartheta_{0}$ ser bijetivo. Além disso, como

$$
\vartheta_{0}\left(v_{J_{1}} \cdot v_{J_{2}}\right)=\vartheta_{0}\left(v_{\left(J_{1}+J_{2}\right)}\right)=v_{\left(J_{1}+J_{2}\right)^{\prime}}=v_{\left(J_{1}^{\prime}+J_{2}^{\prime}\right)}=v_{J_{1}^{\prime}} \cdot v_{J_{2}^{\prime}}=\vartheta_{0}\left(v_{J_{1}}\right) \cdot \vartheta_{0}\left(v_{J_{2}}\right),
$$

concluímos que $\vartheta_{0}$ é um automorfismo de $D\left[\xi_{1}, \xi_{2}, \ldots ; \eta\right]$ como anel e podemos, agora, considerar a sua extensão $\vartheta$ a $D_{H}$, que ainda verifica $\vartheta^{m}=1$.

Seja $t$ uma indeterminada não comutativa. Consideremos o anel de polinômios não comutativos $D_{H}[t ; \vartheta]$, munido da relação

$$
t a=\vartheta(a) t, \forall a \in D_{H}
$$

Afirmamos que o polinômio $t^{m}-\eta$ é irredutível e central em $D_{H}[t ; \vartheta]$. Com efeito,

$$
\left(t^{m}-\eta\right) a=t^{m} a-\eta a=\vartheta^{m}(a) t^{m}-a \eta=a t^{m}-a \eta=a\left(t^{m}-\eta\right),
$$

para todo $a \in D_{H}$ e também, como $\vartheta(\eta)=\eta$ implica $t \eta=\vartheta(\eta) t=\eta t$, temos

$$
t\left(t^{m}-\eta\right)=t t^{m}-t \eta=t^{m} t-\eta t=\left(t^{m}-\eta\right) t,
$$

e estas duas equações acima mostram que $t^{m}-\eta$ é central. Para mostrar que também é irredutível, suponhamos que

$$
t^{m}-\eta=a^{\prime}[t] b^{\prime}[t]=\left(a_{0}^{\prime}+a_{1}^{\prime} t+\ldots a_{r}^{\prime} t^{r}\right)\left(b_{0}^{\prime}+b_{1}^{\prime} t+\cdots+b_{s}^{\prime} t^{s}\right),
$$

em que $a_{i}^{\prime}, b_{j}^{\prime} \in D_{H}$. Então, para cada $i, j$, podemos escrever, usando um denominador comum, digamos $0 \neq c=c[\xi ; \eta] \in D[\xi ; \eta]$,

$$
a_{i}^{\prime}=c^{-1} a_{i}^{\prime \prime} \text { e } b_{j}^{\prime}=c^{-1} b_{j}^{\prime \prime},
$$

em que $a_{i}^{\prime \prime}=a_{i}^{\prime \prime}[\xi ; \eta]$ e $b_{j}^{\prime \prime}=b_{j}^{\prime \prime}[\xi ; \eta]$ estão em $D[\xi ; \eta]$. Se $p$ é um inteiro positivo tal que $\eta^{p}$ divide $c, a_{i}^{\prime \prime}$ e $b_{j}^{\prime \prime}$, digamos $c=\eta^{p} d, a_{i}^{\prime \prime}=\eta^{p} a_{i}$ e $b_{j}^{\prime \prime}=\eta^{p} b_{j}$, para todos $i, j$, então

$$
a_{i}^{\prime}=\left(\eta^{p} d\right)^{-1}\left(\eta^{p} a_{i}\right)=d^{-1} a_{i} \quad \text { e } \quad b_{j}^{\prime}=\left(\eta^{p} d\right)^{-1}\left(\eta^{p} b_{j}\right)=d^{-1} b_{j} .
$$


Tomando $p$ maximal com esta propriedade, concluímos que

$$
d\left(t^{m}-\eta\right)=\left(a_{0}+a_{1} t+\cdots+a_{r} t^{r}\right)\left(b_{0}+b_{1} t+\cdots+b_{s} t^{s}\right)
$$

e $\eta$ não é um fator comum aos polinômios $a_{i}=a_{i}[\xi ; \eta], b_{j}=b_{j}[\xi ; \eta]$. Sejam $k \in\{1, \ldots, r\}$ e $h \in\{1, \ldots, s\}$ os menores possíveis tais que $a_{k} \not \equiv 0(\bmod \eta)$ e $b_{h} \not \equiv 0(\bmod \eta)$. Como $\vartheta(\eta)=\eta$, temos que, para qualquer inteiro $q, \vartheta^{q}$ não altera uma relação de congruência $\bmod \eta$. Em particular,

$\vartheta^{q}\left(a_{k}\right) \not \equiv 0(\bmod \eta), \quad \vartheta^{q}\left(b_{h}\right) \not \equiv 0(\bmod \eta), \quad \vartheta^{q}\left(a_{i}\right) \equiv 0(\bmod \eta) \quad$ e $\quad \vartheta^{q}\left(b_{j}\right) \equiv 0(\bmod \eta)$,

para quaisquer $i<k$ e $j<h$. Computando o membro direito em (2.3), concluímos que os coeficientes de $t^{u}$, com $0 \leq u<k+h$, são múltiplos de $\eta$. Para estudar o coeficiente de $t^{k+h}$, observemos que, para todo $i \in\{0, \ldots, k+h\}$,

$$
\left(a_{i} t^{i}\right)\left(b_{k+h-i} t^{k+h-i}\right)=a_{i} \vartheta^{i}\left(b_{k+h-i}\right) t^{k+h} .
$$

Das congruências acima, resulta que

$$
\sum_{i=0}^{k+h} a_{i} \vartheta^{i}\left(b_{k+h-i}\right) \equiv a_{k} \vartheta^{k}\left(b_{h}\right) \equiv a_{k} b_{h} \not \equiv 0(\bmod \eta) .
$$

Computando o membro esquerdo de (2.3), todos os coeficientes de $t^{i}$, com $i<m$ são múltiplos de $\eta$. Comparando os cômputos de ambos os membros, necessariamente $k+h=$ $m$ e, com maior razão, $d=a_{k} b_{h} \not \equiv 0(\bmod \eta)$. Portanto $k=r$ e $h=s$. Caso ocorresse $r>0$ e $s>0$, teríamos

$$
d \eta=a_{0} b_{0} \equiv 0\left(\bmod \eta^{2}\right) .
$$

Como $d, a_{0}$ e $b_{0}$ estão em $D[\xi ; \eta]$ e $\eta$ é irredutível neste anel, a igualdade $d \eta=a_{0} b_{0}$ não pode ocorrer, visto que $d \not \equiv 0(\bmod \eta)$. Então $r=0$ ou $s=0$, mostrando que $t^{m}-\eta$ é irredutível.

Como $\vartheta$ é um automorfismo de $D_{H}$, é possível usar o algoritmo da divisão para mostrar que os ideais (à esquerda, à direita, bilaterais) de $D_{H}[t ; \vartheta]$ são principais. Então o anel quociente $D_{m}=D_{H}[t ; \vartheta] /\left(t^{m}-\eta\right)$ é uma $C$-álgebra com divisão e verificamos imediatamente que a aplicação de $D$ em $D_{m}$ dada por $d \mapsto d+\left(t^{m}-\eta\right)$ é um monomorfismo de $C$-álgebras.

Para construir $\mathscr{D}_{m}$ e $H_{m}$, observemos que $\vartheta_{H}=\left.\vartheta\right|_{H}$ é um automorfismo de $H$, precisamente o determinado pela aplicação $\xi_{i} \mapsto \xi_{i+1}$, se $1 \leq i \leq m-1$ e $\xi_{m} \mapsto \xi_{1}$. Então o grupo cíclico finito $\Theta=\left\{\vartheta_{H}, \vartheta_{H}^{2}, \ldots, \vartheta_{H}^{m}=1\right\}$ é um subgrupo do grupo de automorfismos de $H$. Seja $H_{m}$ o subcorpo de $H$ fixado por $\Theta$, isto é,

$$
H_{m}=\left\{\lambda \in H \mid \vartheta_{H}^{i}(\lambda)=\lambda, 1 \leq i \leq m\right\}
$$

Claramente, $H_{m}$ é uma extensão de $K$ e resulta que $\operatorname{dim}_{H_{m}} H=m$ (cf. Kaplansky [Kap72], p.16, teorema 11). Outrossim, o fato de $\vartheta_{H}$ ser um automorfismo de $H$ implica 
que $H\left[t ; \vartheta_{H}\right]$ é uma subálgebra de $D_{H}[t ; \vartheta]$. Consideremos

$$
\mathscr{D}_{m}=\frac{H\left[t ; \vartheta_{H}\right]+\left(t^{m}-\eta\right)}{\left(t^{m}-\eta\right)} \cong \frac{H\left[t ; \vartheta_{H}\right]}{H\left[t ; \vartheta_{H}\right] \cap\left(t^{m}-\eta\right)} .
$$

Por argumentos análogos aos do parágrafo anterior, concluímos que $\mathscr{D}_{m}$ é uma $C$-subálgebra com divisão de $D_{m}$, precisamente

$$
\mathscr{D}_{m}=\left\{p[t]+\left(t^{m}-\eta\right) \mid p[t] \in H\left[t ; \vartheta_{H}\right]\right\} .
$$

Usando o algoritmo da divisão, verificamos que, dado $p[t] \in H\left[t ; \vartheta_{H}\right]$, existem únicos $p_{0}, p_{1}, \ldots, p_{m-1} \in H$ tais que

$$
p[t] \equiv p_{0}+p_{1} t+\cdots+p_{m-1} t^{m-1}\left(\bmod t^{m}-\eta\right)
$$

Logo $\operatorname{dim}_{H} \mathscr{D}_{m}=m$ e, conseqüentemente, $\operatorname{dim}_{H_{m}} \mathscr{D}_{m}=m^{2}$.

Para completar esta demonstração, resta provar que $H_{m}$ é o centro de $\mathscr{D}_{m}$. Com efeito, seja $z[t]=z_{0}+z_{1} t+\cdots+z_{m-1} t^{m-1} \in H\left[t ; \vartheta_{H}\right]$ e suponhamos que $z[t]+\left(t^{m}-\eta\right)$ esteja no centro de $\mathscr{D}_{m}$. Necessariamente,

$0 \equiv z[t] \xi_{1}-\xi_{1} z[t] \equiv z_{1}\left(\xi_{2}-\xi_{1}\right) t+z_{2}\left(\xi_{3}-\xi_{1}\right) t^{2}+\cdots+z_{m-1}\left(\xi_{m}-\xi_{1}\right) t^{m-1}\left(\bmod t^{m}-\eta\right)$.

Resulta que $z_{i}\left(\xi_{i+1}-\xi_{1}\right)=0$, para todo $i \in\{1, \ldots, m-1\}$, o que força $z_{1}=\cdots=z_{m-1}=0$. Então $z[t]=z_{0} \in H$ e deve ocorrer, também,

$$
0 \equiv z_{0} t^{i}-t^{i} z_{0} \equiv\left(z_{0}-\vartheta^{i}\left(z_{0}\right)\right) t^{i}\left(\bmod t^{m}-\eta\right)
$$

donde concluímos que $z_{0}=\vartheta^{i}\left(z_{0}\right)$, para todo $i \in\{1, \ldots, m\}$. Logo $z[t]=z_{0} \in H_{m}$. Temos, assim, que o centro de $\mathscr{D}_{m}$ é um subcorpo de $H_{m}$. Como a recíproca é evidente, segue que $\mathscr{D}_{m}$ é uma $H_{m}$-álgebra central, com divisão.

Estamos prontos para enunciar e demonstrar o principal resultado, como segue.

Teorema 2.6.6. Seja $D$ uma C-álgebra com divisão de centro $K$ tal que $\operatorname{dim}_{K} D=$ $\infty$. Então $I(D)=\bigcap_{m=1}^{\infty} I\left(M_{m}(C)\right)$. Em particular, todas as $C$-álgebras com divisão de dimensão infinita sobre seus centros têm as mesmas identidades.

Prova: A demonstração é tratada da seguinte maneira: construiremos uma $C$-álgebra com divisão $\mathscr{D}$ de dimensão infinita sobre o seu centro e de modo a verificar $I(\mathscr{D})=$ $\bigcap_{m=1}^{\infty} I\left(M_{m}(C)\right)$ e uma $C$-álgebra com divisão $E$ que contém ambas $D$ e $\mathscr{D}$. O resultado, portanto, será obtido a partir do lema 2.6.4, segundo o qual $I(D)=I(E)=I(\mathscr{D})$.

Consideremos o conjunto $\mathbb{N}^{*}=\{1,2, \ldots\}$ e as álgebras com divisão $D_{m}$ e $\mathscr{D}_{m}$, $m \in \mathbb{N}^{*}$, obtidas nos moldes do lema 2.6.5. Seja

$$
\mathcal{B}=\left\{m \mathbb{N}^{*} \mid m \in \mathbb{N}^{*}\right\}=\left\{m, 2 m, 3 m, \ldots \mid m \in \mathbb{N}^{*}\right\} \subseteq \mathcal{P}\left(\mathbb{N}^{*}\right)
$$

Claramente, $\mathcal{B} \neq \emptyset$ e, para quaisquer $m_{1}, m_{2} \in \mathbb{N}^{*}$, temos que $m_{1} \mathbb{N}^{*} \neq \emptyset$ e também $m_{1} \mathbb{N}^{*} \cap m_{2} \mathbb{N}^{*} \supseteq\left(m_{1} m_{2}\right) \mathbb{N}^{*}$. Logo $\mathcal{B}$ é base de um filtro em $\mathbb{N}^{*}$ e podemos tomar um 
ultra-filtro $\mathscr{F}$ que contém $\mathcal{B}$. Seja $E=\prod D_{m} / \mathscr{F}$. Então, pelo lema 1.3.7, $E$ é uma $C$-álgebra com divisão. No lema 2.6.5, vimos que $D \hookrightarrow D_{m}$, portanto podemos considerar o monomorfismo de $D$ em $\prod D_{m}$ dado por $d \mapsto f_{d}$, em que $f_{d}(m)=d$, para todo $m \in \mathbb{N}^{*}$. Claramente, $f_{d} \equiv 0(\bmod \mathscr{F})$ se e somente se $d=0$. Assim, temos um monomorfismo $D \hookrightarrow E$.

Também vimos no lema 2.6.5 que $\mathscr{D}_{m} \hookrightarrow D_{m}$, portanto $\prod \mathscr{D}_{m}$ é naturalmente identificada como uma subálgebra de $\prod D_{m}$. Denotemos $\mathscr{D}=\prod \mathscr{D}_{m} / \mathscr{F}$ que, pelo lema 1.3.7, é uma álgebra com divisão. Temos, pois, um monomorfismo $\mathscr{D} \hookrightarrow E$.

De acordo com o lema 2.6.5, cada $\mathscr{D}_{m}$ é uma álgebra central simples, de dimensão finita $m^{2}$ sobre seu centro. Decorre que, pelo teorema 2.5.1, $I\left(\mathscr{D}_{m}\right)=I\left(M_{m}(C)\right)$. Como $\mathbb{N}^{*} \in \mathscr{F}$, segue do teorema 2.3.6 que

$$
I(\mathscr{D}) \supseteq \bigcap_{m=1}^{\infty} I\left(\mathscr{D}_{m}\right)=\bigcap_{m=1}^{\infty} I\left(M_{m}(C)\right) .
$$

Para provar a inclusão contrária, fixemos arbitrários $S \in \mathscr{F}, m \in \mathbb{N}^{*}$ e $p=p\langle x ; y\rangle \in I_{S}=$ $\bigcap_{s \in S} I\left(M_{s}(C)\right)$. Deve ocorrer $S \cap m \mathbb{N}^{*} \neq \emptyset$, digamos que $m n \in S \cap m \mathbb{N}^{*}$, para algum inteiro positivo $n$. Então $p \in I\left(M_{m n}(C)\right)$ e, pelo teorema 2.5.2, obtemos $p \in I\left(M_{m}(C)\right)$. Com maior razão, $I_{S} \subseteq I\left(M_{m}(C)\right)$. Como $S, m$ e $p$ são arbitrários, concluímos que

$$
\bigcup_{S \in \mathscr{F}} I_{S} \subseteq \bigcap_{m=1}^{\infty} I\left(M_{m}(C)\right) .
$$

Pelo teorema 2.3.6, temos $I(\mathscr{D})=\bigcup I_{S}$, logo

$$
I(\mathscr{D}) \subseteq I\left(\bigcap_{m=1}^{\infty} M_{m}(C)\right)
$$

e ambos os conjuntos são, por conseguinte, iguais.

Por fim, observemos que, necessariamente, $\mathscr{D}$ é uma álgebra com divisão de dimensão infinita sobre seu centro. Caso contrário, usaríamos o teorema 2.5.1 para concluir que $I\left(\cap M_{m}(C)\right)=I(\mathscr{D})=I\left(M_{n}(C)\right)$, para algum inteiro positivo $n$, mas isto contradiz o teorema 2.5.2, segundo o qual $I\left(M_{n p}(C)\right) \subsetneq I\left(M_{n}(C)\right)$, para qualquer inteiro $p>1$.

\subsection{Um último teorema sobre identidades racionais}

A esta altura, é razoável indagar: se $r=r\langle x ; y\rangle$ e $s=s\langle x ; y\rangle$ não são identidades racionais de uma $C$-álgebra com divisão, então podemos concluir que $r s$ não é uma identidade racional? Por um lado, podemos afirmar apenas que existem homomorfismos admissíveis $\varphi$ e $\psi$ de $C\langle x ; y\rangle$ em $D$ tais que $\varphi(r) \neq 0 \neq \psi(s)$. Como eventualmente $\varphi$ e $\psi$ são distintos, precisamos encontrar um homomorfismo admissível $\sigma$ que não anule simultaneamente $r$ e $s$, do que concluiremos $\sigma(r s) \neq 0$. A resposta é afirmativa e está contida no próximo teorema, que encerra este capítulo. 
Teorema 2.7.1. Seja $D$ uma $C$-álgebra com divisão qualquer. Então $C\langle x ; y\rangle / I(D)$ é um anel sem divisores de zero e existe um anel com divisão $\mathfrak{D}$ tal que $C\langle x ; y\rangle / I(D) \hookrightarrow \mathfrak{D} e$ $I(\mathfrak{D})=I(D)$.

Prova: Caso $\Im(D)=\emptyset$, temos $I(D)=C\langle x ; y\rangle$ e o resultado segue trivialmente pondo $\mathfrak{D}=D$. Podemos assumir, portanto, que $\mathfrak{I}(D) \neq \emptyset$.

Sejam $r=r\langle x ; y\rangle$ e $s=s\langle x ; y\rangle$ em $C\langle x ; y\rangle$. Suponhamos que nenhum dos dois seja uma identidade racional de $D$. Então devem existir homomorfismos admissíveis $\varphi$ e $\psi$ em $\Im(D)$ tais que $\varphi(r) \neq 0 \neq \psi(s)$. Vamos provar que $r s \not \equiv 0(\bmod I(D))$.

Sejam $u, v$ indeterminadas comutativas. Formemos, nos moldes do lema 1.1.22, com $\alpha=1$, o anel de frações $D_{1}(u)$ a partir de $D[u]$. Consideremos o homomorfismo $\tau$ de $C_{0}$ em $D_{1}(u)$ determinado pela aplicação $x_{i} \mapsto \varphi\left(x_{i}\right) u$. Então $\epsilon_{1}^{u} \tau_{0}=\varphi_{0}$ e, procedendo de maneira inteiramente análoga ao lema 2.6.4, podemos estender $\tau_{0}$ a um homomorfismo admissível $\tau \in \mathfrak{I}\left(D_{1}(u)\right)$ que verifica $\epsilon_{1}^{u} \tau=\varphi$.

Formemos, agora, também nos moldes do lema 1.1.22, com $\alpha=0$, o anel de frações $D(u)_{0}(v)$ a partir de $D_{u}[v]$. Consideremos o homomorfismo $\sigma_{0}$ de $C_{0}$ em $D(u)_{0}(v)$ determinado pela aplicação $x_{i} \mapsto \varphi\left(x_{i}\right) u+\psi\left(x_{i}\right) v$. Então $\epsilon_{0}^{v} \sigma_{0}=\tau_{0}$. Mais uma vez, podemos estender $\sigma_{0}$ a um homomorfismo admissível $\sigma \in \mathfrak{I}\left(D(u)_{0}(v)\right)$ que verifica $\epsilon_{0}^{v} \sigma=\tau$ e, com maior razão,

$$
\epsilon_{1}^{u} \epsilon_{0}^{v} \sigma=\epsilon_{1}^{u} \tau=\varphi
$$

Vamos repetir o mesmo processo dos dois últimos parágrafos. Formamos o anel de frações $D_{1}(v)$ e o homomorfismo $\tau_{0}^{\prime}$ determinado pela aplicação $x_{i} \mapsto \psi\left(x_{i}\right) v$. Então $\epsilon_{1}^{v} \tau_{0}^{\prime}=\psi_{0}$ e podemos estender $\tau_{0}^{\prime}$ a um homomorfismo admissível $\tau^{\prime} \in \mathfrak{I}\left(D_{1}(v)\right)$ que verifica $\epsilon_{1}^{v} \tau^{\prime}=\psi$. Formamos, agora, o anel de frações $D(v)_{0}(u)$, obtido a partir de $D_{v}[u]$, e tomamos $\sigma_{0}^{\prime}$ de $C_{0}$ em $D(v)_{0}(u)$ determinado pela aplicação $x_{i} \mapsto \varphi\left(x_{i}\right) u+\psi\left(x_{i}\right) v$. Então $\epsilon_{0}^{u} \sigma_{0}^{\prime}=\tau_{0}^{\prime}$ e a fortiori obtemos $\sigma^{\prime} \in \mathfrak{I}\left(D_{u}\right)$, extensão de $\sigma_{0}^{\prime}$ que verifica $\epsilon_{0}^{u} \lambda^{\prime}=\tau^{\prime}$. Conseqüentemente,

$$
\epsilon_{1}^{v} \epsilon_{0}^{u} \sigma^{\prime}=\epsilon_{1}^{v} \tau^{\prime}=\psi
$$

Ambos $D(u)_{0}(v)$ e $D(v)_{0}(u)$ são, de acordo com o lema 1.1.22, subanéis de $D(u, v)$, portanto podemos considerar que $\sigma$ e $\sigma^{\prime}$ estão definidos de $C\langle x ; y\rangle$ em $D(u, v)$. Como está claro acima que $\sigma_{0}=\sigma_{0}^{\prime}$, resulta, pelo lema 2.3.4, que $\sigma=\sigma^{\prime}$ e, como assumimos $\varphi(r) \neq 0 \neq \psi(s)$, concluímos que

$$
\sigma(r) \neq 0 \neq \sigma(s)
$$

Como ambos $\sigma(r)$ e $\sigma(s)$ estão no anel com divisão $D(u, v)$, segue que $0 \neq \sigma(r) \sigma(s)=$ $\sigma(r s)$. Logo $r s$ não é uma identidade racional de $D(u, v)$. Conforme o corolário 2.4.2, temos $I(D(u, v))=I(D)$, o que acarreta $r s \notin I(D)$, completando a primeira parte desta demonstração. 
Para demonstrar a segunda parte do teorema, vamos descrever o seguinte diagrama comutativo:

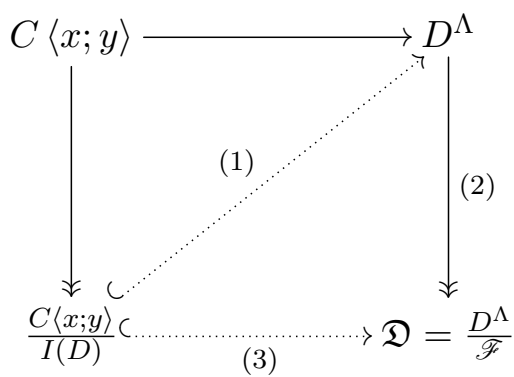

Tomamos, inicialmente, o conjunto $\Lambda=\mathfrak{I}(D)$ dos homomorfismos admissíveis em $D$. Consideremos a aplicação de $C\langle x ; y\rangle$ em $D^{\Lambda}$ dada por $r=r\langle x ; y\rangle \mapsto f_{r}$, em que $f_{r}(\lambda)=\lambda(r)$, para todo $\lambda \in \Lambda$. Então $f_{r}=0$ se e somente se $\lambda(r)=0$ para todo $\lambda$, ou seja, se e somente se $r \in \bigcap_{\lambda \in \Lambda} \operatorname{ker} \lambda=I(D)$. Pelo teorema ho homomorfismo, redunda que a aplicação $\bar{r}=r+I(D) \mapsto f_{r}$ é um monomorfismo (1) de $C\langle x ; y\rangle / I(D)$ em $D^{\Lambda}$ bem definido.

Para cada $r=r\langle x ; y\rangle$ em $C\langle x ; y\rangle$, definimos $S_{r}=\{\lambda \in \Lambda \mid \lambda(r) \neq 0\}$. Consideramos o conjunto

$$
\mathcal{B}=\left\{S_{r} \mid r \notin I(D)\right\}
$$

Como $I(D) \neq C\langle x ; y\rangle$, temos $\mathcal{B} \neq \emptyset$ e é óbvio que $S_{r} \neq \emptyset$ sempre que $r \notin I(D)$. Além disso, se também $s=s\langle x ; y\rangle$ não é uma identidade racional de $D$, temos $S_{s} \in \mathcal{B}$ e a primeira parte da demonstração implica que $r s \notin I(D)$, $\log S_{r s} \in \mathcal{B}$. Assim, para cada $\lambda \in \Lambda$, ocorrerá $0 \neq \lambda(r s)=\lambda(r) \lambda(s)$ se e somente se $\lambda(r) \neq 0 \neq \lambda(s)$. Isto mostra que $S_{r} \cap S_{s}=S_{r s}$. Sucede que $\mathcal{B}$ é base de um filtro em $\Lambda$ e podemos tomar um ultra-filtro $\mathscr{F}$ que contém $\mathcal{B}$. Conseqüentemente, $\left\{\lambda \in \Lambda \mid f_{r}(\lambda) \neq 0\right\}=\{\lambda \in \Lambda \mid \lambda(r) \neq 0\}=S_{r} \in \mathscr{F}$, ou seja, $f_{r} \not \equiv 0(\bmod \mathscr{F})$. Isto mostra que a composição da projeção ao quociente $(2)$ com o monomorfismo (1) é, ainda, um monomorfismo (3). Pelo lema $1.3 .7, \mathfrak{D}=D^{\Lambda} / \mathscr{F}$ é o anel com divisão procurado. 


\section{Capítulo 3}

\section{Aplicações}

\subsection{O grupo multiplicativo de anéis com divisão}

O objetivo desta seção é aplicar a teoria desenvolvida no segundo capítulo para mostrar que o grupo multiplicativo de uma $C$-álgebra com divisão (não comutativa) não satisfaz identidades de grupo não triviais.

Para alcançar o nosso objetivo, vamos mostrar que uma $C$-álgebra de grupo, formada sobre um grupo livre com infinitos geradores, pode ser embutida em uma $C$-álgebra com divisão de dimensão infinita sobre seu centro (portanto não comutativa), e disto resultará o que queremos mostrar.

Antes de estabelecer os resultados básicos desta seção, precisamos observar alguns fatos.

Sejam $\{\xi\}=\left\{\xi_{1}, \xi_{2}, \ldots\right\}, \mathfrak{F}$ o grupo livre gerado por $\{\xi\}$ e $C[\mathfrak{F}]$ a $C$-álgebra de grupo gerada a partir de $\mathfrak{F}$. Fixemos $p=p[\xi] \in C[\mathfrak{F}]$ não-nulo. Então existe um inteiro positivo $n$ tal que $p \in C\left[\mathfrak{F}_{n}\right]$, em que $\mathfrak{F}_{n}$ é o grupo livre gerado por $\left\{\xi_{1}, \ldots, \xi_{n}\right\}$, isto é, $p$ contém apenas as palavras que envolvem $\xi_{1}, \ldots, \xi_{n}$ e seus respectivos inversos. Seja $T: C\left[\mathfrak{F}_{n}\right] \rightarrow C\langle x ; y\rangle$ a transformação $C$-linear obtida a partir da extensão das aplicações $\xi_{j} \mapsto x_{j} x_{n+j}, \xi_{j}^{-1} \mapsto y_{j}$ e $1 \mapsto 1$ aos elementos de $\mathfrak{F}_{n}$, isto é, uma palavra (reduzida) $\xi_{i_{1}}^{\epsilon\left(i_{1}\right)} \cdots \xi_{i_{m}}^{\epsilon\left(i_{m}\right)}$, em que $\epsilon\left(i_{j}\right) \in\{-1,1\}$, é transformada no monômio $z_{i_{1}} \cdots z_{i_{m}}$, em que $z_{i_{j}}=x_{i_{j}} x_{n+i_{j}}$, se $\epsilon\left(i_{j}\right)=1$, e $z_{i_{j}}=y_{i_{j}}$, se $\epsilon\left(i_{j}\right)=-1$. Observemos que $T$ é injetiva e denotemos $p\langle x ; y\rangle=T(p)$, portanto $p\langle x ; y\rangle \neq 0$.

Formemos o $Q$-ideal $I_{0}=\left(q_{1}, \ldots, q_{n}\right)$ de $C\langle x ; y\rangle$, em que $q_{j}=q_{j}\langle x ; y\rangle=y_{j} x_{j} x_{n+j}-$ 1. Então o conjunto de homomorfismos $I_{0}$-admissíveis em $D$ é não vazio: por exemplo, as aplicações de $C\langle x ; y\rangle$ em $D x_{i} \mapsto 1$ e $y_{j} \mapsto 1$, para todos $i \geq 1$ e $1 \leq j \leq n$, determinam um tal homomorfismo. Seja $\varphi \in \mathfrak{I}(D)$ arbitrário. Resulta que

$$
(\varphi T)\left(\xi_{j}\right)=\varphi\left(x_{j} x_{n+j}\right)=\varphi\left(y_{j}\right)^{-1}=\left[(\varphi T)\left(\xi_{j}^{-1}\right)\right]^{-1},
$$

o que acarreta $(\varphi T)\left(\xi_{j}^{-1}\right)=\left[(\varphi T)\left(\xi_{j}\right)\right]^{-1}$. Com maior razão, $\varphi T$ é um $C$-homomorfismo de álgebras. 
O nosso objetivo agora é mostrar que, dada uma $C$-álgebra com divisão $D$ de dimensão infinita sobre seu centro, $p\langle x ; y\rangle$ não é uma identidade $\left(I_{0}-\right)$ racional de $D$. Faremos isso construindo uma $C$-álgebra de dimensão finita - que, portanto, está embutida em um anel de matrizes sobre $C$ - da qual $p\langle x ; y\rangle$ não é uma identidade racional. Resultará que $p\langle x ; y\rangle \notin \bigcap M_{m}(C)$, e a conclusão seguirá pelo teorema 2.6.6.

Inicialmente, observemos que, pelo teorema 2.7.1, $C\langle x ; y\rangle / I(D)$ é um domínio, portanto $y_{j} x_{j} x_{n+j} \equiv 1(\bmod I(D))$ implica que $y_{j}+I(D), x_{j}+I(D)$ e $x_{n+j}+I(D)$ são unidades em $C\langle x ; y\rangle / I(D)$. Isso nos autoriza a formular a seguinte relação:

$$
p\langle x ; y\rangle \equiv \sum_{(i)} c_{(i)}\left(x_{i_{1}} x_{n+i_{1}}\right)^{\epsilon\left(i_{1}\right)} \ldots\left(x_{i_{m}} x_{n+i_{m}}\right)^{\epsilon\left(i_{m}\right)}(\bmod I(D)),
$$

em que $\epsilon\left(i_{j}\right) \in\{-1,1\}$. Podemos ir além e, eliminando os parênteses acima, obtemos

$$
p\langle x ; y\rangle \equiv \sum_{(k)} c_{(k)} x_{k_{1}}^{\epsilon\left(k_{1}\right)} x_{k_{2}}^{\epsilon\left(k_{2}\right)} \ldots x_{k_{2 m}}^{\epsilon\left(k_{2 m}\right)}(\bmod I(D)),
$$

em que

$$
x_{k_{1}}=x_{i_{1}}, x_{k_{2}}=x_{n+i_{1}} \text { e } \epsilon\left(k_{1}\right)=\epsilon\left(k_{2}\right)=1, \text { se } \epsilon\left(i_{1}\right)=1,
$$

$\mathrm{Ou}$

$$
x_{k_{1}}=x_{n+i_{1}}, x_{k_{2}}=x_{i_{1}} \text { e } \epsilon\left(k_{1}\right)=\epsilon\left(k_{2}\right)=-1, \text { se } \epsilon\left(i_{1}\right)=-1,
$$

e assim por diante. Outrossim, como os monômios de $p\langle x ; y\rangle$ foram obtidos a partir de palavras reduzidas, concluímos que $k_{j} \neq k_{j+1}$.

Sejam $\{v\}=\left\{v_{1}, \ldots, v_{2 n}\right\}$ um conjunto de indeterminadas não comutativas, $C\langle v\rangle$ a álgebra livre com unidade gerada por $\{v\}$, e $\mu, \nu$ inteiros positivos quaisquer. Formemos o ideal (bilateral) $J$ de $C\langle v\rangle$, gerado pelos elementos da forma $v_{j}^{\mu+1}$, com $j \in\{1, \ldots, 2 n\}$ e $v_{j_{1}} v_{j_{2}} \cdots v_{j_{\nu}}$, com $j_{k} \in\{1, \ldots, 2 n\}$ e $1 \leq k \leq \nu$. Como os monômios de grau não inferior a $\nu$ e as potências de cada $v_{j}$ não inferiores a $\mu+1$ são congruentes a zero, concluímos que o conjunto finito

$$
\left\{v_{j_{1}} v_{j_{2}} \cdots v_{j_{\nu-1}} \mid j_{k} \in\{1, \ldots, 2 n\}, 1 \leq k \leq \nu-1\right\} \cup\left\{v_{j}^{m} \mid 1 \leq j \leq 2 n, m \leq \mu\right\}
$$

é gerador da $C$-álgebra $B=C\langle v\rangle / J$, o que acarreta $\operatorname{dim}_{C} B<\infty$. Para cada $j$, denotemos $b_{j}=1-v_{j}$ e $b_{j}^{\prime}=1+v_{j}+\cdots+v_{j}^{\mu}$. Então

$$
b_{j} b_{j}^{\prime} \equiv b_{j}^{\prime} b_{j} \equiv 1(\bmod J) .
$$

Consideremos o homomorfismo $\psi$ de $C\langle x ; y\rangle$ em $C\langle v\rangle$ determinado pelas aplicações $x_{j} \mapsto$ $b_{j}, x_{n+j} \mapsto b_{n+j}$ e $y_{j} \mapsto b_{n+j}^{\prime} b_{j}^{\prime}, 1 \leq j \leq n$, e $x_{j} \mapsto 0$, se $j>2 n$. Então

$$
\psi\left(q_{j}\right)=\psi\left(y_{j} x_{j} x_{n+j}-1\right)=b_{n+j}^{\prime} b_{j}^{\prime} b_{j} b_{n+j}-1 \equiv 0(\bmod J)
$$

para todo $j$, o que mostra que $\rho \psi \in \mathfrak{I}(B)$, em que $\rho: C\langle v\rangle \rightarrow B$ é a projeção canônica ao quociente. Além disso, de acordo com a relação (3.1), temos que

$$
\psi(p\langle x ; y\rangle)=p\left\langle b ; b^{\prime}\right\rangle \equiv \sum_{(k)} c_{(k)} b_{k_{1}}^{\epsilon\left(k_{1}\right)} b_{k_{2}}^{\epsilon\left(k_{2}\right)} \ldots b_{k_{2 m}}^{\epsilon\left(k_{2 m}\right)}(\bmod J),
$$


ou ainda

$$
\psi(p\langle x ; y\rangle)=p\left\langle b ; b^{\prime}\right\rangle=\sum_{(k)} c_{(k)}\left(1+\cdots+\epsilon_{(k)} v_{k_{1}}^{\delta\left(k_{1}\right)} v_{k_{2}}^{\delta\left(k_{2}\right)} \cdots v_{k_{2 m}}^{\delta\left(k_{2 m}\right)}\right),
$$

em que $\delta\left(k_{j}\right)=1$, se $\epsilon\left(k_{j}\right)=1, \delta\left(k_{j}\right)=\mu$, se $\epsilon\left(k_{j}\right)=-1$, e $\epsilon_{(k)} \in\{-1,1\}$. Denotemos

$$
v_{(k)}^{\delta_{(k)}}=\epsilon_{(k)} v_{k_{1}}^{\delta\left(k_{1}\right)} v_{k_{2}}^{\delta\left(k_{2}\right)} \cdots v_{k_{2 m}}^{\delta\left(k_{2 m}\right)} \quad \text { e } \quad p_{(k)}(b)=1+\cdots+v_{(k)}^{\delta_{(k)}}
$$

Observemos que $v_{(k)}^{\delta_{(k)}}$ é o monômio em $v$ de maior grau em $p_{(k)}(b)$, a saber $r_{(k)}+s_{(k)} \mu$, em que $r_{(k)}$ é o número de $k_{j}$ 's tais que $\epsilon\left(k_{j}\right)=1$ e $s_{(k)}$ é o número de $k_{j}$ 's tais que $\epsilon\left(k_{j}\right)=-1$. Além disso, como $k_{j} \neq k_{j+1}$, as potências de cada $v_{k_{j}}$ em $v_{(k)}^{\delta_{(k)}}$ não superam $\mu$.

Se todos os $s_{(k)}$ 's são zero, então $p\langle x ; y\rangle$ não envolve as indeterminadas $y_{j}$, ou seja, $p\langle x ; y\rangle=p\langle x\rangle$ não pode ser uma identidade polinomial em $D$, porquanto este tem dimensão infinita sobre seu centro. Podemos assumir, portanto, que, para algum $(k)$, temos $s_{(k)} \geq 1$. Tomemos $r=\max _{(k)} r_{(k)}$. Observemos que este máximo independe dos inteiros $\mu, \nu$, de modo que podemos escolher $\mu>r$. Assim, se $r_{(i)}+s_{(i)} \mu=r_{(j)}+s_{(j)} \mu$, temos $r_{(i)}=\left(s_{(j)}-s_{(i)}\right) \mu+r_{(j)}$, com $r_{(j)}<\mu$. Como também $r_{(i)}<\mu$, segue que, usando a unicidade do resto e do quociente no algoritmo da divisão para os números inteiros, $r_{(i)}=r_{(j)}$ e $s_{(i)}=s_{(j)}$. Agora escolhemos $\nu>\max _{(k)}\left(r_{(k)}+s_{(k)} \mu\right)$. Nestes moldes, concluímos que $v_{(k)}^{\delta_{(k)}} \notin J$, para todo $(k)$.

Podemos reformular a equação (3.2), destacando os monômios em $v$ de maior grau, como segue:

$$
p\left\langle b ; b^{\prime}\right\rangle=\tilde{p}\left\langle b ; b^{\prime}\right\rangle+\sum_{(k)} c_{(k)} v_{(k)}^{\delta_{(k)}},
$$

e para provar que $p\langle x ; y\rangle$ não é uma identidade racional de $B$, é suficiente mostrar que $\sum_{(k)} c_{(k)} v_{(k)}^{\delta_{(k)}} \not \equiv 0(\bmod J)$. Com efeito, fixemos um monômio $v_{(i)}^{\delta_{(i)}}$ de grau máximo $r_{(i)}+s_{(i)} \mu$. Se $v_{(j)}^{\delta_{(j)}}$ também possui grau máximo, decorre das observações acima que $r_{(j)}=r_{(i)}$ e $s_{(j)}=s_{(i)}$ e, como $(i)$ e $(j)$ não possuem índices consecutivos iguais, estes monômios coincidem se e somente se $(i)=(j)$.

Concluímos que os monômios em $v$ de grau máximo aparecem uma única vez em $p\left\langle b ; b^{\prime}\right\rangle$, não possuem potências de $v_{j}$ maiores que $\mu$ e seus graus são menores que $\nu$, do que resulta, a fortiori,

$$
(\rho \psi)(p\langle x ; y\rangle)=\rho\left(p\left\langle b ; b^{\prime}\right\rangle\right) \neq 0
$$

como queríamos.

Proposição 3.1.1. Seja $\Lambda=\{\lambda\}$ a coleção de todos os C-homomorfismos de álgebra de $C[\mathfrak{F}]$ em uma $C$-álgebra com divisão $D$ de dimensão infinita sobre seu centro. Então $\bigcap_{\lambda \in \Lambda} \operatorname{ker} \lambda=(0)$

Prova: Seja $p=p[\xi]$ não nulo em $C[\mathfrak{F}]$. Conforme os argumentos acima, existe um homomorfismo admissível $\varphi$ de $C\langle x ; y\rangle$ em $D$ tal que $(\varphi T)(p)=\varphi(p\langle x ; y\rangle) \neq 0$. Isto prova a afirmação. 
Teorema 3.1.2. A álgebra de grupo $C[\mathfrak{F}]$ não possui divisores de zero e pode ser embutida em um anel com divisão de dimensão infinita sobre seu centro.

Prova: Sejam $D$ uma $C$-álgebra com divisão de dimensão infinita sobre seu centro $K$, $p=p[\xi]$ e $q=q[\xi]$ ambos não nulos em $C[\mathfrak{F}]$. De acordo com os argumentos e as notações do início deste capítulo, existe um $Q$-ideal $I_{0}$ de $C\langle x ; y\rangle$ tal que $\mathfrak{I}(D) \neq \emptyset$ e nem $p\langle x ; y\rangle=$ $T(p)$, nem $q\langle x ; y\rangle=T(q)$ são identidades $\left(I_{0}-\right)$ racionais de $D$. Pelo teorema 2.7.1, resulta que $p\langle x ; y\rangle q\langle x ; y\rangle$ também não é uma identidade racional de $D$. Seja $\varphi \in \mathfrak{I}(D)$ tal que $\varphi(p\langle x ; y\rangle q\langle x ; y\rangle) \neq 0$. Portanto

$$
(\varphi T)(p q)=(\varphi T)(p)(\varphi T)(q)=\varphi(p\langle x ; y\rangle) \varphi(q\langle x ; y\rangle)=\varphi(p\langle x ; y\rangle q\langle x ; y\rangle) \neq 0
$$

do que concluímos, necessariamente, $p q \neq 0$, o que prova a primeira afirmação.

Para demonstrar o restante, vamos descrever o seguinte diagrama comutativo:

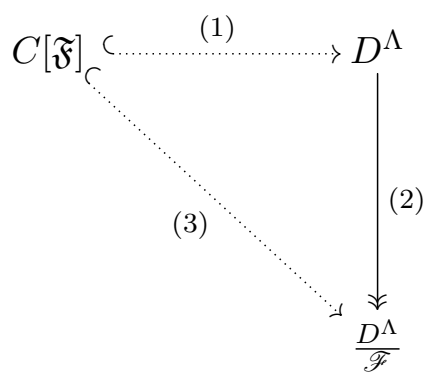

Seja $\Lambda=\{\lambda\}$ a coleção de todos os $C$-homomorfismos de álgebra de $C[\mathfrak{F}]$ em uma $C$-álgebra com divisão $D$, de dimensão infinita sobre seu centro. Decorre da proposição 3.1.1 que a aplicação $p[\xi] \mapsto f_{p}$ de $C[\mathfrak{F}]$ em $D^{\Lambda}$, em que $f_{p}(\lambda)=\lambda(p)$, é um monomorfismo (1) de $C$-álgebras.

Para cada $p=p[\xi]$ em $C[\mathfrak{F}]$, definimos $S_{p}=\{\lambda \in \Lambda \mid \lambda(p) \neq 0\}$. Consideremos o conjunto $\mathcal{B}=\left\{S_{p} \mid 0 \neq p \in C[\mathfrak{F}]\right\}$. Se $p, q \in C[\mathfrak{F}]$ são não nulos, então $S_{p} \neq \emptyset \neq S_{q}$ e, como $C[\mathfrak{F}]$ não tem divisores de zero e o contradomínio de cada $\lambda$ é o anel com divisão $D$, resulta que $S_{p} \cap S_{q}=S_{p q} \in \mathcal{B}$. Logo $\mathcal{B}$ é base de um filtro, portanto podemos tomar um ultra-filtro $\mathscr{F}$ em $\Lambda$ que contém $\mathcal{B}$. Nestes moldes, como $S_{p} \in \mathscr{F}$, necessariamente $f_{p} \not \equiv 0(\bmod \mathscr{F})$ e concluímos que a composição da projeção ao quociente $(2)$ com o monomorfismo (1) é, ainda, um monomorfismo (3) de $C[\mathfrak{F}]$ em $D^{\Lambda} / \mathscr{F}$. Este último é uma $C$-álgebra com divisão de acordo com o lema 1.3.7. Outrossim, como $D \hookrightarrow D^{\Lambda} / \mathscr{F}$, usamos as observações da seção 2.1 do segundo capítulo para concluir que $D^{\Lambda} / \mathscr{F}$ tem dimensão infinita sobre seu centro.

O principal resultado desta seção é dado a seguir.

Teorema 3.1.3. Seja $D$ uma $C$-álgebra com divisão de centro $K$. Então o grupo multiplicativo $D^{*}$ não satisfaz qualquer identidade de grupo não trivial.

Prova: Suponhamos que $W(x)=x_{i_{1}}^{\epsilon\left(i_{1}\right)} \cdots x_{i_{n}}^{\epsilon\left(i_{n}\right)}$, com $\epsilon\left(i_{j}\right) \in\{-1,1\}$, seja uma identidade em $D^{*}$. Então $W(x)-1$ é uma identidade racional de $D . \operatorname{Se} \operatorname{dim}_{K} D=\infty$, então 
segue do teorema 2.6.6 que $W(x)-1$ é uma identidade racional de todas as $C$-álgebras com divisão de dimensão infinita sobre seus centros. Em particular, deve ser uma identidade da álgebra com divisão que contém o grupo livre $\mathfrak{F}$. Então $W(x)=1$.

Suponhamos que $\operatorname{dim}_{K} D<\infty$. Então, pelo teorema 2.5.1, $W(x)-1 \in I(D)=$ $I\left(M_{n}(C)\right)=I\left(M_{n}(\bar{C})\right)$, em que $\bar{C}$ é o fecho algébrico de $C$. Vamos mostrar, em um primeiro estágio, que $W(x)-1$ não é uma identidade racional de $M_{2}(\bar{C})$. Com efeito, fazendo $x_{i_{j}}=u^{i_{j}} v u^{i_{j}}$, a palavra $W(x)$ se reescreve como uma palavra em duas indeterminadas, digamos

$$
\begin{aligned}
W\left(u^{i} v u^{i}\right) & =\left(u^{i_{1}} v u^{i_{1}}\right)^{\epsilon\left(i_{1}\right)} \cdots\left(u^{i_{n}} v u^{i_{n}}\right)^{\epsilon\left(i_{n}\right)} \\
& =u^{n_{k}} v^{m_{k}} u^{n_{k-1}} v^{m_{k-1}} \cdots u^{n_{1}} v^{m_{1}} u^{n_{0}}=W^{(k)}(u, v)
\end{aligned}
$$

Sejam arbitrários $c$ e $d$ não nulos em $\bar{C}-\{1\}$, s e $t$ indeterminadas comutativas. Fazemos

$$
U=\left[\begin{array}{ll}
1 & 0 \\
s & c
\end{array}\right] \text { e } V=\left[\begin{array}{ll}
1 & t \\
0 & d
\end{array}\right], \quad \log 0 \quad U^{n}=\left[\begin{array}{cc}
1 & 0 \\
c_{[n]} s & c^{n}
\end{array}\right] \text { e } \quad V^{m}=\left[\begin{array}{cc}
1 & d_{[m]} t \\
0 & d^{m}
\end{array}\right],
$$

em que

$$
c_{[n]}=\left(c^{n}-1\right)(c-1)^{-1} \text { e } d_{[m]}=\left(d^{m}-1\right)(d-1)^{-1},
$$

para quaisquer inteiros não nulos $n$ e $m$. Nestes moldes, temos

$$
U^{n} V^{m}=\left[\begin{array}{cc}
1 & d_{[m]} t \\
c_{[n]} s & \left(c^{n} d^{m}+c_{[n]} d_{[m]} s t\right)
\end{array}\right] .
$$

Vamos analisar, agora, as entradas da matriz $W^{(k)}(U, V)=\left(W_{i j}^{(k)}(U, V)\right)=\left(W_{i j}^{(k)}\right)$. Começamos com

$$
W^{(0)}=U^{n_{0}}=\left[\begin{array}{cc}
1 & 0 \\
c_{\left[n_{0}\right]} s & c^{n_{0}}
\end{array}\right]
$$

e denotamos $\alpha_{0}=1$ e $\beta_{0}=c_{\left[n_{0}\right]}$. Prosseguindo, temos

$$
W^{(1)}=U^{n_{1}} V^{m_{1}} U^{n_{0}}=\left[\begin{array}{cc}
\left(1+c_{\left[n_{0}\right]} d_{\left[m_{1}\right]} s t\right) & (\cdots) \\
\left(c_{\left[n_{1}\right]} s+c_{\left[n_{0}\right]} c^{n_{1}} d^{m_{1}} s+c_{\left[n_{0}\right]} c_{\left[n_{1}\right]} d_{\left[m_{1}\right]} s^{2} t\right) & (\cdots)
\end{array}\right],
$$

portanto, denotando $\alpha_{1}=c_{\left[n_{0}\right]} d_{\left[m_{1}\right]}$ e $\beta_{1}=c_{\left[n_{0}\right]} c_{\left[n_{1}\right]} d_{\left[m_{1}\right]}$, temos

$$
W_{11}^{(1)}=\alpha_{1} s t+1 \text { e } W_{21}^{(1)}=\beta_{1} s^{2} t+\cdots .
$$

Procedendo indutivamente, para $1<j \leq k$, temos $W^{(j)}=\left(U^{n_{j}} V^{m_{j}}\right) W^{(j-1)}$, e resulta que

$$
W_{11}^{(j)}=W_{11}^{(j-1)}+d_{\left[m_{j}\right]} t W_{21}^{(j-1)}=d_{\left[m_{j}\right]} t \cdot \beta_{j-1} s^{j} t^{j-1}+\alpha_{j-1}(s t)^{j-1}+\cdots,
$$

e também

$$
\begin{aligned}
W_{21}^{(j)} & =c_{\left[n_{j}\right]} s W_{11}^{(j-1)}+\left(c^{n_{j}} d^{m_{j}}+c_{\left[n_{j}\right]} d_{\left[m_{j}\right]} s t\right) W_{21}^{(j-1)} \\
& =c_{\left[n_{j}\right]} d_{\left[m_{j}\right]} s t \cdot \beta_{j-1} s^{j} t^{j-1}+c_{\left[n_{j}\right]} s \cdot \alpha_{j-1}(s t)^{j-1}+\cdots
\end{aligned}
$$


A partir das equações acima, concluímos que $\alpha_{j}=d_{\left[m_{j}\right]} \cdot \beta_{j-1}$ e que $\beta_{j}=c_{\left[n_{j}\right]} d_{\left[m_{j}\right]} \beta_{j-1}$. Com maior razão, resulta que

$$
\beta_{k}=\left(c_{\left[n_{0}\right]} c_{\left[n_{1}\right]} \cdots c_{\left[n_{k}\right]}\right)\left(d_{\left[m_{1}\right]} d_{\left[m_{2}\right]} \cdots d_{\left[m_{k}\right]}\right) .
$$

Como $\bar{C}$ é infinito, podemos escolher devidamente $c$, de modo que não seja uma raiz da unidade de ordem $n_{i}$, para cada $i \in\{0, \ldots, k\}$, e $d$, de modo que não seja uma raiz da unidade de ordem $m_{i}$, para cada $i \in\{1, \ldots, k\}$. Nestes moldes, obtemos $\beta \neq 0$, e necessariamente

$$
W_{21}^{(k)}=W_{21}^{(k)}(s, t)=\beta_{k} s^{k+1} t^{k}+\cdots \in \bar{C}(s, t)
$$

é um polinômio não nulo e seu monômio de maior grau é, precisamente, $\beta_{k} s^{k+1} t^{k}$. Basta agora fazer $s=a$ e $t=b$, em que $a, b \in \bar{C}$ são tais que $W_{21}^{(k)}(a, b) \neq 0$, e isto é possível, porque $\bar{C}$ é infinito. Tomamos, finalmente, as matrizes invertíveis

$$
A=\left[\begin{array}{ll}
1 & 0 \\
a & c
\end{array}\right] \text { e } B=\left[\begin{array}{ll}
1 & b \\
0 & d
\end{array}\right]
$$

e obtemos $W^{(k)}(A, B) \neq 1$. Para um $n>2$ qualquer, tomamos as matrizes (dadas em forma de bloco)

$$
\bar{A}=\left[\begin{array}{cc}
A & 0 \\
0 & 1
\end{array}\right] \text { e } \bar{B}=\left[\begin{array}{cc}
B & 0 \\
0 & 1
\end{array}\right]
$$

em que 1 é um bloco identidade de tamanho $(n-2) \times(n-2)$. Ainda assim, $W(\bar{A}, \bar{B})^{(k)} \neq 1$, o que completa a demonstração.

Em particular, concluímos um resultado já existente (Hua [Hua50]):

Corolário 3.1.4. O grupo multiplicativo de um anel com divisão de centro infinito não é solúvel.

\section{2 $\mathrm{O}$ anel de funções racionais em um anel com divisão}

Nesta seção, vamos usar os resultados dos capítulos anteriores para, a partir de uma $C$-álgebra com divisão arbitrária $D$, construir um anel $C_{D}(x)$ de funções racionais em $D$. Vamos mostrar que, entre outras propriedades, $C_{D}(x)$ é um anel com divisão cuja estrutura algébrica depende apenas da dimensão de $D$ sobre seu centro, isto é, se duas $C$ álgebras com divisão têm a mesma dimensão sobre seus centros, então os anéis de funções racionais de cada uma são isomorfos.

Inicialmente, vamos expandir os conceitos apresentados no segundo capítulo. Até aqui, estudamos as identidades racionais de uma $C$-álgebra com divisão $D$ relativas a um $Q$-ideal da álgebra livre com unidade $C\langle x ; y\rangle=C\left\langle x ; y_{1}, \ldots, y_{n}\right\rangle$. Podemos sempre tomar um número finito de indeterminadas $y_{j}$, porquanto os polinômios da álgebra livre sempre envolvem finitas variáveis $x_{i}$ e $y_{j}$. Para generalizar as idéias expostas anteriormente, vamos tomar, em um primeiro estágio, ambos os conjuntos $\{x\}=\left\{x_{1}, x_{2}, \ldots\right\}$ e 
$\{y\}=\left\{y_{1}, y_{2}, \ldots\right\}$ infinitos. Formamos, a seguir, a coleção $\mathcal{Q}$ de todos os possíveis $Q$-ideais (os quais têm, por definição, nível finito) em $C\langle x ; y\rangle$. Claramente, $\mathcal{Q}$ é um subconjunto do conjunto das partes de $C\langle x ; y\rangle$, portanto pode ser indexado biunivocamente por um conjunto $A=\{\alpha\}$. Nestes moldes, tendo em consideração que apenas finitas indeterminadas $y_{j}$ estão envolvidas nos geradores de cada $Q$-ideal, para cada $\alpha$ em $A$, estão determinados:

1. Uma subálgebra $C\left\langle x ; y_{\alpha}\right\rangle=C\left\langle x ; y_{\alpha 1}, \ldots, y_{\alpha n_{\alpha}}\right\rangle$ de $C\langle x ; y\rangle$.

2. Um $Q$-ideal $I_{\alpha}^{0}$ de $C\left\langle x ; y_{\alpha}\right\rangle$, gerado por polinômios $q_{\alpha j}=q_{\alpha j}\left\langle x ; y_{\alpha}\right\rangle=y_{\alpha j} p_{\alpha j}-1$, em que $p_{\alpha j}=p_{\alpha j}\left\langle x ; y_{\alpha 1}, \ldots, y_{\alpha j-1}\right\rangle$.

3. Um conjunto $\mathfrak{I}_{\alpha}(D)$ (eventualmente vazio) de homomorfismos $I_{\alpha}^{0}$-admissíveis.

4. Um conjunto $I_{\alpha}(D)$ das identidades $I_{\alpha}^{0}$-racionais de $D$.

Para os nossos propósitos, vamos considerar apenas os $Q$-ideais para os quais os conjuntos de homomorfismos admissíveis não são vazios. Sem perda de generalidade, poderemos prosseguir com as notações $\mathcal{Q}$ e $A=\{\alpha\}$ para fazer referência a esses objetos. Enfatizamos que $\mathcal{Q}$ não é vazio: a seção anterior provê exemplos de $Q$-ideais para os quais existem homomorfismos admissíveis.

Para cada $\alpha$ em $A$, denotaremos $R_{\alpha}=C\left\langle x ; y_{\alpha}\right\rangle / I_{\alpha}(D)$. Conforme as nossas convenções, $\mathfrak{I}_{\alpha}(D)$ é não vazio, o que acarreta $I_{\alpha}(D) \neq C\left\langle x ; y_{\alpha}\right\rangle$, ou seja, $R_{\alpha}$ é não trivial e, de acordo com o teorema 2.7.1, é também um domínio. Os próximos passos que nos conduzirão à construção de $C_{D}(x)$ consistem em mostrar que os $R_{\alpha}$ 's dispõem-se em um conjunto direcionado. Nosso ponto de partida é a seguinte definição: dados $\alpha$ e $\beta$ em $A$, diremos que um $C$-homomorfismo $\eta: C\left\langle x ; y_{\alpha}\right\rangle \rightarrow C\left\langle x ; y_{\beta}\right\rangle$ é um homomorfismo padrão se $\eta\left(x_{i}\right)=x_{i}$, para todo $i$, e se $\eta\left(I_{\alpha}^{0}\right) \subseteq I_{\beta}^{0}$. Nestes moldes, para qualquer $\varphi$ em $\mathfrak{I}_{\beta}(D)$, temos

$$
\eta\left(I_{\alpha}^{0}\right) \subseteq I_{\beta}^{0} \subseteq \operatorname{ker} \varphi
$$

do que decorre

$$
I_{\alpha}^{0} \subseteq \operatorname{ker}(\varphi \eta)
$$

Sucede que $\varphi \eta \in \mathfrak{I}_{\alpha}(D)$ e, com maior razão,

$$
\mathfrak{I}_{\beta}(D) \eta \subseteq \mathfrak{I}_{\alpha}(D)
$$

Resulta também que, se $p=p\left\langle x ; y_{\alpha}\right\rangle \in C\left\langle x ; y_{\alpha}\right\rangle$ é uma identidade $\left(I_{\alpha}^{0}-\right)$ racional de $D$, deve ocorrer

$$
\varphi(\eta(p))=(\varphi \eta)(p)=0,
$$

donde $\eta(p) \in I_{\beta}(D)$ e, como $p$ e é arbitrário, temos

$$
\eta\left(I_{\alpha}(D)\right) \subseteq I_{\beta}(D) .
$$

Das observações acima, decorre que está bem definido o homomorfismo $\tilde{\eta}: R_{\alpha} \rightarrow R_{\beta}$, de maneira que, para qualquer $\bar{p}=p\left\langle x ; y_{\alpha}\right\rangle+I_{\alpha}(D)$ em $R_{\alpha}$, temos $\tilde{\eta}(\bar{p})=\eta\left(p\left\langle x ; y_{\alpha}\right\rangle\right)+I_{\beta}(D)$. 
Diremos que $\tilde{\eta}$ é o homomorfismo induzido por $\eta$. Ilustramos estas informações no seguinte diagrama (comutativo):

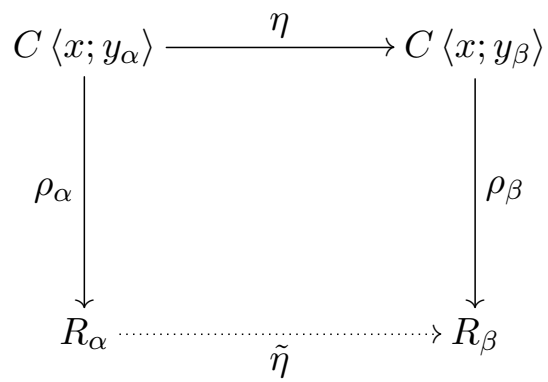

Como de uso, $\rho_{\alpha}$ e $\rho_{\beta}$ denotam as projeções ao quociente. Mais à frente, descreveremos propriedades importantes dos homomorfismos padrão e de seus respectivos homomorfismos induzidos.

Antes de seguir para o próximo lema, estabeleceremos algumas notações. $\Lambda=\{\lambda\}$ é o conjunto de todos os $C$-homomorfismos de $C\langle x\rangle$ em $D$. Recordemos que $C_{0}$ denota a álgebra livre com unidade $C\langle x\rangle$ e que $\varphi_{0}$ denota a restrição de $\varphi$ a $C_{0}$. Para cada $\alpha \mathrm{em}$ $A$, a projeção de $\mathfrak{I}_{\alpha}(D)$ em $\Lambda$ é o conjunto $\widetilde{\mathfrak{I}}_{\alpha}(D)=\left\{\varphi_{0} \mid \varphi \in \mathfrak{I}_{\alpha}(D)\right\}$, que, de acordo com as nossas convenções acima, é não vazio. O lema 2.3.4 nos permite afirmar que $\mathfrak{I}_{\alpha}(D)$ está em correspondência biunívoca com sua projeção em $\Lambda$.

Lema 3.2.1. Para quaisquer $\alpha$ e $\beta$ em $A, \widetilde{\mathfrak{I}}_{\alpha}(D) \cap \widetilde{\mathfrak{I}}_{\beta}(D) \neq \emptyset$.

Prova: Fixemos $\varphi \in \mathfrak{I}_{\alpha}(D)$ e $\psi \in \mathfrak{I}_{\beta}(D)$. Sejam $u$ e $v$ indeterminadas comutativas sobre $D$. Vamos repetir o processo empregado na demonstração do teorema 2.7.1.

Inicialmente, obtemos um homomorfismo admissível $\tau \in \mathfrak{I}_{\alpha}(D(u))$ que possui as propriedades

$$
\tau\left(x_{i}\right)=\varphi\left(x_{i}\right) u, \quad \tau\left(C\left\langle x ; y_{\alpha}\right\rangle\right) \subseteq D_{1}(u) \quad \text { e } \quad \epsilon_{1}^{u} \tau=\varphi .
$$

Depois, obtemos um homomorfismo admissível $\sigma \in \mathfrak{I}_{\alpha}(D(u, v))$ que verifica as propriedades

$$
\sigma\left(x_{i}\right)=\varphi\left(x_{i}\right) u+\psi\left(x_{i}\right) v, \quad \sigma\left(C\left\langle x ; y_{\alpha}\right\rangle\right) \subseteq D(u)_{0}(v) \quad \text { e } \quad \epsilon_{0}^{v} \sigma=\tau,
$$

do que resulta

$$
\epsilon_{1}^{u} \epsilon_{0}^{v} \sigma=\epsilon_{1}^{u} \tau=\varphi
$$

Repetindo o mesmo processo para $\psi$, obteremos $\tau^{\prime} \in \mathfrak{I}_{\beta}(D(v))$ que possui as propriedades

$$
\tau^{\prime}\left(x_{i}\right)=\psi\left(x_{i}\right) v, \quad \tau^{\prime}\left(C\left\langle x ; y_{\beta}\right\rangle\right) \subseteq D_{1}(v) \quad \text { e } \quad \epsilon_{1}^{v} \tau^{\prime}=\psi,
$$

e, em seguida, $\sigma^{\prime} \in \mathfrak{I}_{\alpha}(D(u, v))$, que verifica

$$
\sigma^{\prime}\left(x_{i}\right)=\varphi\left(x_{i}\right) u+\psi\left(x_{i}\right) v, \quad \sigma^{\prime}\left(C\left\langle x ; y_{\beta}\right\rangle\right) \subseteq D(v)_{0}(u) \quad \text { e } \quad \epsilon_{0}^{u} \sigma^{\prime}=\tau^{\prime},
$$


conseqüentemente

$$
\epsilon_{1}^{v} \epsilon_{0}^{u} \sigma^{\prime}=\epsilon_{1}^{v} \tau^{\prime}=\psi
$$

Relembremos que $D_{u}=D(u)$ e que $D_{u}[v]$ é um domínio de ideais principais - o que permite estabelecer a noção de máximo divisor comum. Além disso, como $D(u, v) \cong D_{u}(v)$, podemos escrever, para cada $i \in\left\{1, \ldots, n_{\alpha}\right\}$ e para cada $j \in\left\{1, \ldots, n_{\beta}\right\}$,

$$
\sigma\left(p_{\alpha i}\right)=f_{\alpha i}^{u}[v] g_{\alpha i}^{u}[v]^{-1} \text { e } \sigma^{\prime}\left(p_{\beta j}\right)=h_{\beta j}^{u}[v] k_{\beta j}^{u}[v]^{-1},
$$

em que $f_{\alpha i}^{u}, g_{\alpha i}^{u}, h_{\beta j}^{u}$ e $h_{\beta j}^{u}$ são polinômios em $v$ com coeficientes em $D_{u}$ e estas representações estão dadas de forma irredutível, isto é, um divisor comum de $f_{\alpha i}^{u}$ e de $g_{\alpha i}^{u}$ é uma unidade em $D_{u}[v]$, e $h_{\beta j}^{u}$ e $k_{\beta j}^{u}$ têm a mesma propriedade. Configura-se, a partir disso, a seguinte conseqüência: como $C$ é infinito, podemos fixar $d$ em $C$ tal que $f_{\alpha i}^{u}[d], g_{\alpha i}^{u}[d]$, $h_{\beta j}^{u}[d]$ e $h_{\beta j}^{u}[d]$ são todos não nulos e quaisquer representações na forma irredutível devem verificar a mesma condição. De fato, suponhamos, por exemplo, que, para algum $i$, ocorra $\sigma\left(p_{\alpha i}\right)=p_{\alpha i}^{u}[v] q_{\alpha i}^{u}[v]^{-1}$. De acordo com o lema 1.1.22, o conjunto

$$
S_{d}=\left\{q[v] \in D_{u}[v] \mid q[d] \neq 0\right\}
$$

verifica a condição de Ore (D2). Logo existem $a=a[v]$ e $b=b[v]$ em $D_{u}[v]$ tais que $b[d] \neq 0$ e $g_{\alpha i}^{u} a=q_{\alpha i}^{u} b$, o que claramente implica $f_{\alpha i}^{u} a=p_{\alpha i}^{u} b$. Da primeira das equações, resulta que $q_{\alpha i}^{u}[d]=0$ se e somente se $a[d]=0$; da segunda delas, que $p_{\alpha i}^{u}[d]=0$ se e somente se $a[d]=0$. Então a condição $p_{\alpha i}^{u}[d]=0$ é equivalente a $q_{\alpha i}^{u}[d]=0$. conseqüentemente, se $p_{\alpha i}^{u}[v] q_{\alpha i}^{u}[v]^{-1}$ está na forma irredutível, concluímos que ambos $p_{\alpha i}^{u}$ e $q_{\alpha i}^{u}$ estão em $S_{d}$.

Os fatos expostos no parágrafo acima nos permitem afirmar que estão bem definidos os homomorfismos

$$
\epsilon_{d}^{v} \sigma: C\left\langle x ; y_{\alpha}\right\rangle \rightarrow D_{u} \text { e } \epsilon_{d}^{v} \sigma^{\prime}: C\left\langle x ; y_{\beta}\right\rangle \rightarrow D_{u}
$$

Repetindo a mesma argumentação, usando as representações irredutíveis dos elementos $\left(\epsilon_{d}^{v} \sigma\right)\left(p_{\alpha i}\right),\left(\epsilon_{d}^{v} \sigma^{\prime}\right)\left(p_{\beta j}\right)$ e um conveniente $c$ em $C$, de modo que $\left(\epsilon_{d}^{v} \sigma\right)\left(p_{\alpha i}\right)[c]$ e $\left(\epsilon_{d}^{v} \sigma^{\prime}\right)\left(p_{\beta j}\right)[c]$ existam e sejam distintos de zero, concluímos que estão bem definidos os homomorfismos

$$
\Phi=\epsilon_{c}^{u} \epsilon_{d}^{v} \sigma: C\left\langle x ; y_{\alpha}\right\rangle \rightarrow D \text { e } \Psi=\epsilon_{c}^{u} \epsilon_{d}^{v} \sigma^{\prime}: C\left\langle x ; y_{\beta}\right\rangle \rightarrow D
$$

os quais são, manifestamente, admissíveis. Como $\Phi\left(x_{i}\right)=\varphi\left(x_{i}\right) u+\psi\left(x_{i}\right) v=\Psi\left(x_{i}\right)$, concluímos que $\Phi_{0}=\Psi_{0} \in \widetilde{\mathfrak{I}}_{\alpha}(D) \cap \widetilde{\mathfrak{I}}_{\beta}(D)$, o que completa a prova do lema.

Para mostrar que $\left\{R_{\alpha} \mid \alpha \in A\right\}$ é um conjunto direcionado, precisamos, inicialmente, exibir uma relação binária que seja reflexiva e transitiva. Faremos uso dos homomorfismos padrão, como segue.

Lema 3.2.2. Sejam $\alpha$ e $\beta$ em A quaisquer. Se $\eta: C\left\langle x ; y_{\alpha}\right\rangle \rightarrow C\left\langle x ; y_{\beta}\right\rangle$ é um homomorfismo padrão, então $\tilde{\eta}$ é um monomorfismo e é o único que verifica a relação $\tilde{\eta} \rho_{\alpha}=\rho_{\beta} \eta$. Além disso, se $\xi: C\left\langle x ; y_{\alpha}\right\rangle \rightarrow C\left\langle x ; y_{\beta}\right\rangle$ é um outro homomorfismo padrão, então $\tilde{\xi}=\tilde{\eta}$. 
Prova: Precisamos provar que $\operatorname{ker}\left(\rho_{\beta} \eta\right)=I_{\alpha}(D)$ e, pelo teorema do homomorfismo, obteremos as propriedades enunciadas para $\eta$. Com efeito, suponhamos que $p=p\left\langle x ; y_{\alpha}\right\rangle$ em $C\left\langle x ; y_{\alpha}\right\rangle$ não seja uma identidade racional de $D$, digamos que $\varphi \in \mathfrak{I}_{\alpha}(D)$ seja tal que $\varphi(p) \neq 0$. Tomemos indeterminadas distintas $y_{\gamma 1}, \ldots, y_{\gamma n_{\gamma}}$, de modo que $n_{\gamma}=n_{\alpha}+1 \mathrm{e}$ consideremos a aplicação $\theta: C\left\langle x ; y_{\alpha}\right\rangle \rightarrow C\left\langle x ; y_{\gamma}\right\rangle$ determinada pelas condições $\theta\left(x_{i}\right)=x_{i}$, para todo $i$ e $\theta\left(y_{\alpha j}\right)=y_{\gamma j}$, para todo $j \in\{1, \ldots, n\}$. Manifestamente, $\theta$ é um monomorfismo (é um isomorfismo entre as $C$-álgebras $C\left\langle x ; y_{\alpha}\right\rangle$ e $C\left\langle x ; y_{\gamma 1}, \ldots, y_{\gamma n_{\gamma-1}}\right\rangle$ ), de modo que ainda temos $\theta(p) \neq 0$. Formemos o $Q$-ideal

$$
I_{\gamma}^{0}=\left(\theta\left(q_{\alpha 1}\right), \ldots, \theta\left(q_{\alpha n_{\alpha}}\right), y_{\gamma n_{\gamma}} \theta(p)-1\right)
$$

em que os $q_{\alpha j}$ 's são os geradores do $Q$-ideal $I_{\alpha}^{0}$. Nestes moldes, $\mathfrak{I}_{\alpha}(D) \neq \emptyset$ e, assim, $\gamma \in A$. Com efeito, consideramos o homomorfismo $\psi: C\left\langle x ; y_{\gamma}\right\rangle \rightarrow D$ determinado pelas condições $\psi\left(x_{i}\right)=\varphi\left(x_{i}\right)$ para todo $i, \psi\left(y_{\gamma j}\right)=\varphi\left(y_{\alpha j}\right)$ para todo $j \in\left\{1, \ldots, n_{\gamma}-1=n_{\alpha}\right\}$ e $\psi\left(y_{\gamma n_{\gamma}}\right)=\varphi(p)^{-1}$. Das duas primeiras condições, concluímos que $\psi(\theta(p))=\varphi(p)$; com maior razão, $I_{\gamma}^{0} \subseteq$ ker $\psi$, ou seja, $\psi$ é um homomorfismo admissível. Observemos, ainda, que $\theta$ é um homomorfismo padrão. Podemos, agora, usar o lema 3.2.1 para encontrar $\Phi \in \mathfrak{I}_{\gamma}(D)$ e $\Psi \in \mathfrak{I}_{\beta}(D)$ tais que $\Phi_{0}=\Psi_{0}$. Resulta que $\Phi \theta$ e $\Psi \eta$ estão ambos em $\mathfrak{I}_{\alpha}(D)$ e, como $\eta\left(x_{i}\right)=x_{i}=\theta\left(x_{i}\right)$, temos $(\Phi \theta)\left(x_{i}\right)=\Phi\left(x_{i}\right)=\Psi\left(x_{i}\right)=(\Psi \eta)\left(x_{i}\right)$, o que acarreta $\Phi \theta=\Psi \eta$, pelo lema 2.3.4. Redunda que

$$
(\Psi \eta)(p)=(\Phi \theta)(p)=\Phi\left(y_{\gamma n_{\gamma}}\right)^{-1} \neq 0,
$$

e necessariamente $\eta(p) \notin I_{\beta}(D)$, ou seja, $\left(\rho_{\beta} \eta\right)(p) \neq 0$, o que prova a nossa afirmação.

Para demonstrar o restante do lema, suponhamos que $\xi$ esteja nas condições do enunciado. Então $\xi\left(x_{i}\right)=x_{i}$ e temos, para qualquer $\tau \in \mathfrak{I}_{\beta}(D)$,

$$
(\tau \xi)\left(x_{i}\right)=\tau\left(x_{i}\right)=(\tau \eta)\left(x_{i}\right),
$$

do que decorre, de acordo com o lema 2.3.4, $\tau \xi=\tau \eta \in \mathfrak{I}_{\alpha}(D)$. Dado $q=q\left\langle x ; y_{\alpha}\right\rangle$ qualquer em $I_{\alpha}(D)$, temos, portanto, $0=(\tau \xi)(q)-(\tau \eta)(q)=\tau(\xi(p)-\eta(q))$ e, como $\tau$ foi tomado arbitrariamente, concluímos que $\xi(q) \equiv \eta(q)\left(\bmod I_{\beta}(D)\right)$, mostrando que $\tilde{\xi}\left(q+I_{\alpha}(D)\right)=\tilde{\eta}\left(q+I_{\alpha}(D)\right)$. Como $q$ é qualquer, segue que $\tilde{\xi}=\tilde{\eta}$, como queríamos.

Sejam $\alpha$ e $\beta$ em $A$ quaisquer. À luz do precedente lema, quando existe um homomorfismo padrão $\eta$ de $C\left\langle x ; y_{\alpha}\right\rangle$ em $C\left\langle x ; y_{\beta}\right\rangle$, então $R_{\alpha}$ é isomorfo ao subanel $\eta\left(R_{\alpha}\right)$ de $R_{\beta}$, e tal relação independe de $\eta$. Podemos seguramente identificar $R_{\alpha}$ como um subanel de $R_{\beta}$, neste caso. Reciprocamente, diremos que $R_{\alpha}$ é subanel de $R_{\beta}$ se existir um monomorfismo induzido por um homomorfismo padrão. Como de costume, utilizaremos o símbolo $\hookrightarrow$ para denotar essa relação, que é reflexiva e transitiva. O próximo lema essencialmente no item $\mathbf{2}$ - contém a última propriedade que precisamos validar para $\hookrightarrow$, a fim de mostramos que $\left\{R_{\alpha} \mid \alpha \in A\right\}$ é um conjunto direcionado.

Lema 3.2.3. Sejam $\alpha$ e $\beta$ em A quaisquer. São verdadeiras as seguintes afirmações: 
1. Se $R_{\alpha} \hookrightarrow R_{\beta}$, então $\widetilde{\mathfrak{I}}_{\alpha}(D) \supseteq \widetilde{\mathfrak{I}}_{\beta}(D)$.

2. Existe $\gamma$ em A tal que $R_{\alpha} \hookrightarrow R_{\gamma}, R_{\beta} \hookrightarrow R_{\gamma}$ e $\widetilde{\mathfrak{I}}_{\gamma}(D)=\widetilde{\mathfrak{I}}_{\alpha}(D) \cap \widetilde{\mathfrak{I}}_{\beta}(D)$.

3. Se $p=p\left\langle x ; y_{\alpha}\right\rangle$ em $C\left\langle x ; y_{\alpha}\right\rangle$ não é uma identidade racional de $D$, então, para algum $\gamma$ em $A$, temos $R_{\alpha} \hookrightarrow R_{\gamma}$ e $p+I_{\gamma}(D)$ é uma unidade em $R_{\gamma}$.

Prova: Para provar 1, começamos com um homomorfismo padrão $\eta$ de $C\left\langle x ; y_{\alpha}\right\rangle$ em $C\left\langle x ; y_{\beta}\right\rangle$ e consideramos a aplicação de $\mathfrak{I}_{\beta}(D)$ em $\mathfrak{I}_{\alpha}(D)$ dada por $\varphi \mapsto \varphi \eta$. Se $\varphi^{\prime} \eta=\varphi \eta$, então, como $\eta\left(x_{i}\right)=x_{i}$, temos $\varphi_{0}^{\prime}=\varphi_{0}$. Segue do lema 2.3.4 que $\varphi^{\prime}=\varphi$ e a aplicação em tela é, pois, injetiva. Por outro lado, se $\eta^{\prime}$ é um outro homomorfismo padrão de $C\left\langle x ; y_{\alpha}\right\rangle$ em $C\left\langle x ; y_{\beta}\right\rangle$, então $(\varphi \eta)\left(x_{i}\right)=\left(\varphi \eta^{\prime}\right)\left(x_{i}\right)$, do que resulta, usando mais uma vez o lema 2.3.4, $\varphi \eta=\varphi \eta^{\prime}$. Portanto independe do homomorfismo padrão que tomarmos a maneira como identificamos $\mathfrak{I}_{\beta}(D)$ como subconjunto de $\mathfrak{I}_{\alpha}(D)$. Finalmente, como ambos estão em correspondência biunívoca com suas projeções, obtemos o resultado.

Para provar 2, tomemos indeterminadas distintas $y_{\gamma 1}, \ldots, y_{\gamma n_{\gamma}}$, de modo que $n_{\gamma}=$ $n_{\alpha}+n_{\beta}$ e consideremos as aplicações $\eta_{\alpha}: C\left\langle x ; y_{\alpha}\right\rangle \rightarrow C\left\langle x ; y_{\gamma}\right\rangle$ e $\eta_{\beta}: C\left\langle x ; y_{\beta}\right\rangle \rightarrow C\left\langle x ; y_{\gamma}\right\rangle$, determinadas pelas condições $\eta_{\alpha}\left(x_{i}\right)=x_{i}=\eta_{\beta}\left(x_{i}\right)$, para todo $i, \eta_{\alpha}\left(y_{\alpha j}\right)=y_{\gamma j}$, em que $1 \leq j \leq n_{\alpha}$, e $\eta_{\beta}\left(y_{\beta j}\right)=y_{\gamma n_{\alpha}+j}$, em que $1 \leq j \leq n_{\beta}$. Está claro que $\eta_{\alpha}$ e $\eta_{\beta}$ são monomorfismos. Formemos o $Q$-ideal $I_{\gamma}^{0}$ de $C\left\langle x ; y_{\gamma}\right\rangle$ gerado pelos polinômios $\eta_{\alpha}\left(q_{\alpha 1}\right), \ldots, \eta_{\alpha}\left(q_{\alpha n_{\alpha}}\right), \eta_{\beta}\left(q_{\beta 1}\right), \ldots, \eta_{\beta}\left(q_{\beta n_{\beta}}\right)$. Sejam $\Phi \in \mathfrak{I}_{\alpha}(D)$ e $\Psi \in \mathfrak{I}_{\beta}(D)$ tais que $\Phi_{0}=\Psi_{0}$, de acordo com o lema 3.2.1. Seja $\tau$ o homomorfismo de $C\left\langle x ; y_{\gamma}\right\rangle$ em $D$ determinado pelas aplicações

$$
x_{i} \mapsto \Phi\left(x_{i}\right)=\Psi\left(x_{i}\right), \quad y_{\gamma i} \mapsto \Phi\left(y_{\alpha i}\right) \quad \text { e } \quad y_{\gamma n_{\alpha}+j} \mapsto \Psi\left(y_{\beta j}\right),
$$

com $i \in\left\{1, \ldots, n_{\alpha}\right\}$ e $j \in\left\{1, \ldots, n_{\beta}\right\}$. Nestas condições, $\tau$ é admissível, $\operatorname{logo} \mathfrak{I}_{\gamma}(D) \neq \emptyset$ e, assim, $\gamma \in A$. É imediata a verificação de que $\eta_{\alpha}$ e $\eta_{\beta}$ são homomorfismos padrão, o que prova a primeira parte desta afirmação, com auxílio do lema 3.2.2. Para mostrar o restante, observemos que $\tau_{0}=\left.\tau\right|_{C_{0}}=\Phi_{0}=\Psi_{0}$, do que obtemos $\widetilde{\mathfrak{I}}_{\gamma}(D) \supseteq \widetilde{\mathfrak{I}}_{\alpha}(D) \cap \widetilde{\mathfrak{I}}_{\beta}(D)$. A inclusão contrária a esta última é facilmente obtida por meio da aplicação da afirmação 1.

Finalmente, se um polinômio $p=p\left\langle x ; y_{\alpha}\right\rangle \in C\left\langle x ; y_{\alpha}\right\rangle$ não é uma identidade racional de $D$, construímos $C\left\langle x ; y_{\gamma}\right\rangle, \theta$ e $I_{\gamma}^{0}$, exatamente como foi feito na demonstração da primeira parte do lema 3.2.2. Disto sucederá, com o auxílio do teorema 2.7.1, que $p+I_{\gamma}(D)$ (identificamos $p \operatorname{com} \theta(p)$, de acordo com as observações após o lema 3.2.2) é uma unidade em $R_{\gamma}$, provando 3 .

Uma importante conseqüência da afirmação $\mathbf{2}$ do lema 3.2 .3 é o seguinte

Corolário 3.2.4. O conjunto $\mathcal{B}=\left\{\widetilde{\mathfrak{I}}_{\alpha}(D) \mid \alpha \in A\right\}$ é base de um filtro em $\Lambda$.

Para referências mais adiante, observemos também que, na prova da afirmação $\mathbf{2}$ do lema 3.2.3, podemos, sem perda de generalidade, assumir que $\left\{y_{\alpha}\right\} \cap\left\{y_{\beta}\right\}=\emptyset$ e, 
em vez de construir $C\left\langle x ; y_{\gamma}\right\rangle$ e usar os homomorfismos $\eta_{\alpha}$ e $\eta_{\beta}$, fazemos simplesmente $C\left\langle x ; y_{\gamma}\right\rangle=C\left\langle x ; y_{\alpha}, y_{\beta}\right\rangle$ e os homomorfismos padrão como sendo as inclusões naturais de $C\left\langle x ; y_{\alpha}\right\rangle$ e de $C\left\langle x ; y_{\beta}\right\rangle$ em $C\left\langle x ; y_{\alpha}, y_{\beta}\right\rangle$.

Apresentaremos, a seguir, a mais importante conseqüência dos resultados até agora expostos nesta seção.

Teorema 3.2.5. Dada uma $C$-álgebra com divisão $D$, existe um anel com divisão $C_{D}(x)$ contendo cada $R_{\alpha}$, com $\mathfrak{I}_{\alpha}(D) \neq \emptyset$. Ademais se $\{x\} \subseteq\{z\}$, então existe um monomorfismo natural de $C_{D}(x)$ em $C_{D}(z)$.

Prova: Seja $C_{D}(x)=\bigcup_{\alpha \in A} R_{\alpha}$. Dados quaisquer $\bar{p}, \bar{q} \in C_{D}(x)$, existem $\alpha$ e $\beta$ em $A$ tais que $\bar{p}=p\left\langle x ; y_{\alpha}\right\rangle+I_{\alpha}(D) \in R_{\alpha}$ e $\bar{q}=q\left\langle x ; y_{\beta}\right\rangle+I_{\beta}(D) \in R_{\beta}$. Pela parte 2 do lema 3.2.3, ambos $\bar{p}$ e $\bar{q}$ estão em $R_{\gamma}$, para algum $\gamma$ em $A$. Então podemos tomar $\bar{p}+\bar{q}$ e $\bar{p} \bar{q}$ como elementos de $R_{\gamma}$. Se também ocorre $\bar{p}, \bar{q} \in R_{\delta}$ para algum $\delta$ em $A$, usamos mais uma vez a parte 2 do lema acima para encontrar $\epsilon$ em $A$ tal que $R_{\gamma} \hookrightarrow R_{\epsilon}$ e $R_{\delta} \hookrightarrow R_{\epsilon}$, do que resulta $\bar{p}+\bar{q}$ e $\bar{p} \bar{q}$ estarem determinados do mesmo modo em $R_{\gamma}$ e em $R_{\delta}$ como estão em $R_{\epsilon}$. Isto mostra que a aritmética está bem definida em $C_{D}(x)$ e automaticamente este verifica os axiomas de um anel com unidade. Segue da parte 3 do lema 3.2.3 que $C_{D}(x)$ é um anel com divisão.

Para provar a segunda parte, basta observar que, procedendo da mesma maneira empregada na demonstração do lema 3.2.2, para cada $\alpha$ em $A$, a $C$-álgebra livre $C\left\langle x ; y_{\alpha}\right\rangle$ é naturalmente embutida em $C\left[z ; y_{\alpha}\right]$, de modo que $I_{\alpha}^{0}$ é gerado pelos mesmos polinômios $q_{\alpha j}$ tanto em $C\left\langle x ; y_{\alpha}\right\rangle$ como em $C\left[z ; y_{\alpha}\right]$. A fortiori, teremos um monomorfismo de $R_{\alpha}^{x}=$ $C\left\langle x ; y_{\alpha}\right\rangle / I_{\alpha}^{x}(D)$ em $R_{\alpha}^{z}=C\left[z ; y_{\alpha}\right] / I_{\alpha}^{z}(D)$ para cada $\alpha$ e sucede disto que $C_{D}(x)=\bigcup R_{\alpha}^{x}$ pode ser embutido em $C_{D}(z)=\bigcup R_{\alpha}^{z}$.

Antes de enunciar e provar uma porção de propriedades de $C_{D}(x)$, façamos algumas observações pertinentes. Manifestamente, existe um homomorfismo natural de $C\langle x\rangle$ em $C_{D}(x)$. Por um lado, se $D$ é uma $C$-álgebra com divisão de dimensão infinita sobre seu centro, então $C\langle x\rangle$ está, na verdade, embutido em $C_{D}(x)$, porquanto $D$ não verifica relações polinomiais. Além disso, neste caso, $C_{D}(x)$ é gerado pelos elementos de $C\langle x\rangle$ : para cada $\alpha$ em $A, R_{\alpha}=C\left\langle x ; y_{\alpha}\right\rangle / I_{\alpha}(D)$ é um domínio, conforme o teorema 2.7.1, e cada gerador do $Q$-ideal $I_{\alpha}^{0}$ é da forma $q_{\alpha j}=y_{\alpha j} p_{\alpha j}-1$, com $p_{\alpha j} \in C\left\langle x ; y_{\alpha 1}, \ldots, y_{\alpha j-1}\right\rangle$. Então

$$
y_{\alpha 1} \equiv p_{\alpha 1}\langle x\rangle^{-1}\left(\bmod I_{\alpha}(D)\right), \ldots, y_{\alpha n_{\alpha}} \equiv \cdots \equiv p_{\alpha n_{\alpha}}\left\langle x ; \ldots, p_{\alpha n_{\alpha}-1}^{-1}\right\rangle^{-1}\left(\bmod I_{\alpha}(D)\right),
$$

ou seja, os elementos de $R_{\alpha}$ serão dados a partir dos elementos de $C\langle x\rangle$, envolvendo adição, multiplicação e, eventualmente, inversos. Isto mostra que $C_{D}(x)$ é gerado por $C\langle x\rangle$ envolvendo estas três operações.

Por outro lado, se $D$ é uma $C$-álgebra com divisão de dimensão $n^{2}$ finita sobre seu centro, então, de acordo com o teorema 2.5.1, $I_{\alpha}(D)=I_{\alpha}\left(M_{n}(C)\right)$, para todo $\alpha$ em $A$ e, com maior razão, as identidades polinomiais de $D$ são as mesmas de $M_{n}(C)$ - denotaremos o conjunto dessas identidades por $\mathscr{M}_{n}$. Pelo teorema $2.1 .3, \mathscr{M}_{n}$ é um ideal não trivial de 
$C\langle x\rangle$. Resulta que a imagem de $C\langle x\rangle$ em $C_{D}(x)$ é, neste caso, $C\langle x\rangle / \mathscr{M}_{n}$. Observemos que, do modo como construímos $C_{D}(x)$, os polinômios $p_{\alpha j}$ envolvidos nos geradores do $Q$-ideal $I_{\alpha}^{0}$ não são identidades polinomiais de $D$, porque exigimos $\mathfrak{I}_{\alpha}(D) \neq \emptyset$ e, neste caso, se $\varphi$ é um homomorfismo admissível de $C\left\langle x ; y_{\alpha}\right\rangle$ em $D$, necessariamente $\varphi\left(p_{\alpha j}\right) \neq 0$. Então as relações de congruência entre os $y_{\alpha j}$ 's e os $p_{\alpha j}$ 's módulo $I_{\alpha}(D)$ são as mesmas apreciadas acima, ainda que a dimensão de $D$ sobre seu centro seja finita. Em outras palavras, $C_{D}(x)$ é, também neste caso, um anel com divisão gerado pela imagem de $C\langle x\rangle$.

Em [Ami55], Amitsur mostrou que $C\langle x\rangle / \mathscr{M}_{n}$ é um domínio de Ore (à direita e à esquerda), ou seja, que gera, a menos de isomorfismos, um único anel com divisão que, ademais, é uma álgebra central simples de dimensão $n^{2}$. Em virtude da discussão no parágrafo acima e do citado resultado, concluímos que, no caso em que $D$ é uma álgebra com divisão de dimensão $n^{2}$ finita sobre seu centro, então $C_{D}(x)$ é isomorfo ao anel total de frações de $C\langle x\rangle / \mathscr{M}_{n}$ e, portanto, uma álgebra com divisão de dimensão $n^{2}$ sobre seu centro.

Temos motivação suficiente para enunciar e demonstrar o próximo

Teorema 3.2.6. Se $D$ e $\mathfrak{D}$ são $C$-álgebras com divisão de dimensões infinitas (ou finitas e iguais) sobre seus centros, então existe uma identificação natural entre $C_{D}(x)$ e $C_{\mathfrak{D}}(x)$. Ademais, se $D$ tem dimensão $n^{2}$ finita, então $C_{D}(x)$ é isomorfo ao anel total de frações de $C\langle x\rangle / \mathscr{M}_{n}$, que é um domínio de Ore. Se as dimensões de $D$ e de $\mathfrak{D}$ não são iguais, então $C_{D}(x)$ e $C_{\mathfrak{D}}(x)$ não são isomorfos.

Prova: Decorre dos teoremas 2.5.1 e 2.6.6 que, se as dimensões de $D$ e de $\mathfrak{D}$ são infinitas ou são finitas e iguais, então $I_{\alpha}(D)=I_{\alpha}(\mathfrak{D})$, para qualquer $\alpha$ em $A$. Com maior razão, identificamos $R_{\alpha}=C\left\langle x ; y_{\alpha}\right\rangle / I_{\alpha}(D)$ com $R_{\alpha}^{\prime}=C\left\langle x ; y_{\alpha}\right\rangle / I_{\alpha}(\mathfrak{D})$ e, a fortiori, obtemos a identificação entre $C_{D}(x)=\bigcup R_{\alpha}$ e $C_{\mathfrak{D}}(x)=\bigcup R_{\alpha}^{\prime}$. Isto prova a primeira das afirmações.

A segunda afirmação decorre das observações previamente feitas. Outrossim, se as dimensões de $D$ e de $\mathfrak{D}$ são finitas, entretanto distintas, concluímos que $C_{D}(x)$ e $C_{\mathfrak{D}}(x)$ não são isomorfos, porquanto são álgebras centrais simples de dimensões diferentes.

Finalmente, se $D$ tem dimensão infinita sobre seu centro, então $C\langle x\rangle \hookrightarrow C_{D}(x)$, de acordo com o que discutimos acima. Sucede que, por conter uma subálgebra livre, $C_{D}(x)$ não verifica relação polinomial alguma e, assim, tem dimensão infinita sobre seu centro. Se, por outro lado, $\mathfrak{D}$ tem dimensão finita, então $C_{D}(x)$ e $C_{\mathfrak{D}}(x)$ não são isomorfos. Isto conclui a demonstração.

Sucede imediatamente deste último teorema o seguinte

Corolário 3.2.7. $\bar{p} \in C_{D}(x)$ é nulo se e somente se, para qualquer $\alpha \in A$ tal que $\bar{p} \in R_{\alpha} e$ para qualquer $p\left\langle x ; y_{\alpha}\right\rangle \in C\left\langle x ; y_{\alpha}\right\rangle$ tal que $\bar{p}=p\left\langle x ; y_{\alpha}\right\rangle+I_{\alpha}(D), p\left\langle x ; y_{\alpha}\right\rangle$ é uma identidade racional de qualquer $C$-álgebra com divisão $\mathfrak{D}$ tal que $C_{D}(x) \cong C_{\mathfrak{D}}(x)$.

Podemos, ainda, relacionar ultra-produtos e o anel $C_{D}(x)$.

Teorema 3.2.8. $C_{D}(x)$ pode ser embutido em um ultra-produto de cópias de $D$. 
Prova: Seja $\Lambda=\lambda$ o conjunto de todos os homomorfismos de $C\langle x\rangle$ em $D$. Em conformidade com o corolário 3.2.4, tomamos um ultra-filtro em $\Lambda$ que contém a base $\mathcal{B}$. Vamos provar que $C_{D}(x) \hookrightarrow D^{\Lambda} / \mathscr{F}$.

Inicialmente, definimos uma relação de $C_{D}(x)$ em $D^{\Lambda}$ : para cada $\bar{p}$ em $C_{D}(x)$, diga$\operatorname{mos} \bar{p}=p\left\langle x ; y_{\alpha}\right\rangle+I_{\alpha}(D) \in R_{\alpha}$ para algum $\alpha$ em $A$, tomamos a função $f_{\bar{p}}^{\alpha}: \Lambda \rightarrow D$ dada por $f_{\bar{p}}^{\alpha}(\lambda)=0$, se $\lambda \notin \widetilde{\mathfrak{I}}_{\alpha}(D)$ e, se $\lambda \in \widetilde{\mathfrak{I}}_{\alpha}(D)$, então $\lambda=\varphi_{0}$, para um único $\varphi \in \mathfrak{I}_{\alpha}(D)$, e definimos $f_{\bar{p}}^{\alpha}(\lambda)=\varphi\left(p\left\langle x ; y_{\alpha}\right\rangle\right)$. Observemos que, se $p\left\langle x ; y_{\alpha}\right\rangle \equiv q\left\langle x ; y_{\alpha}\right\rangle\left(\bmod I_{\alpha}(D)\right)$, então $\varphi(p)=\varphi(q)$. Redunda que $f_{\bar{p}}^{\alpha}(\lambda)=\varphi(p)=\varphi(q)=f_{\bar{q}}^{\alpha}(\lambda)$ e isto é suficiente para afirmar que $f_{\bar{p}}^{\alpha}$ está bem definida. Para sermos breves, podemos simplesmente escrever $\varphi(\bar{p})$. Se também $\bar{p} \in R_{\beta}$ para outro $\beta$ em $A$, é possível tomar $f_{\bar{p}}^{\beta}$ de maneira análoga, mas eventualmente ocorrerá $f_{\bar{p}}^{\alpha} \neq f_{\bar{p}}^{\beta}$. Para contornar esse obstáculo, vamos usar a projeção $\rho$ de $D^{\Lambda}$ ao quociente $D^{\Lambda} / \mathscr{F}$ e provar que $\rho\left(f_{\bar{p}}^{\alpha}\right)=\rho\left(f_{\bar{p}}^{\beta}\right)$ para quaisquer $\alpha$ e $\beta$ para os quais temos $\bar{p} \in R_{\alpha}$ e $\bar{p} \in R_{\beta}$.

Em virtude do lema 3.2.3, existe $\gamma$ em $A$ tal que $R_{\alpha} \hookrightarrow R_{\gamma}$ e $R_{\beta} \hookrightarrow R_{\gamma}$, com $\widetilde{\mathfrak{I}}_{\gamma}(D)=\widetilde{\mathfrak{I}}_{\alpha}(D) \cap \widetilde{\mathfrak{I}}_{\beta}(D)$ e, pelo corolário 3.2.4, temos ainda que $\widetilde{\mathfrak{I}}_{\gamma}(D) \in \mathscr{F}$. Dado $\lambda$ em $\widetilde{\mathfrak{I}}_{\gamma}(D)$, digamos que $\lambda=\psi_{0}$, para um único $\psi$ em $\mathfrak{I}_{\gamma}(D)$, então $\psi_{\alpha}=\left.\psi\right|_{C\left\langle x ; y_{\alpha}\right\rangle} \in \mathfrak{I}_{\alpha}(D) \mathrm{e}$ $\psi_{\beta}=\left.\psi\right|_{C\left\langle x ; y_{\beta}\right\rangle} \in \mathfrak{I}_{\beta}(D)$. Logo

$$
f_{\bar{p}}^{\alpha}(\lambda)=\psi_{\alpha}(\bar{p})=\psi(\bar{p})=\psi_{\beta}(\bar{p})=f_{\bar{p}}^{\beta}(\lambda)
$$

e então $\left\{\lambda \in \Lambda \mid f_{\bar{p}}^{\alpha}(\lambda)=f_{\bar{p}}^{\beta}(\lambda)\right\} \supseteq \widetilde{\mathfrak{I}}_{\gamma}(D) \in \mathscr{F}$. conseqüentemente, $f_{\bar{p}}^{\alpha} \equiv f_{\bar{p}}^{\beta}(\bmod \mathscr{F})$, como queríamos.

Seja $F: C_{D}(x) \rightarrow D^{\Lambda} / \mathscr{F}$ a aplicação que cumpre $F(\bar{p})=\rho\left(f_{\bar{p}}^{\alpha}\right)$. Cômputos simples e rotineiros mostram que $F$ é um homomorfismo não trivial. Como ambos $C_{D}(x)$ e $D^{\Lambda} / \mathscr{F}$ são anéis com divisão, necessariamente $F$ é um monomorfismo, e a demonstração está completa.

Teorema 3.2.9. Todo automorfismo de $C\langle x\rangle$ estende-se de modo único a um automorfismo de $C_{D}(x)$.

Prova: Seja $\eta: C\langle x\rangle \rightarrow C\langle x\rangle$ um automorfismo. Fixemos $\alpha$ em $A$ e consideremos a álgebra livre com unidade $C\left\langle x ; y_{\alpha}\right\rangle$ e o $Q$-ideal $I_{\alpha}^{0}=\left(q_{\alpha 1}, \ldots, q_{\alpha n_{\alpha}}\right)$, com $q_{\alpha j}=y_{\alpha j} p_{\alpha j}-1$. Formemos uma nova álgebra livre $C\left\langle x ; y_{\alpha^{\prime}}\right\rangle$, com $n_{\alpha^{\prime}}=n_{\alpha}$, e o $Q$-ideal $I_{\alpha^{\prime}}^{0}$ gerado pelos polinômios da forma $q_{\alpha^{\prime} j}=y_{\alpha^{\prime} j} p_{\alpha^{\prime} j}\left\langle\eta(x) ; y_{\alpha^{\prime} 1}, \ldots, y_{\alpha^{\prime} j-1}\right\rangle-1$, em que $p_{\alpha^{\prime} j}$ é obtido a partir do polinômio $p_{\alpha j}$ pela aplicação de $\eta$ às indeterminadas $x_{i}$, com $1 \leq j \leq n_{\alpha^{\prime}}$.

Seja $\eta_{\alpha}: C\left\langle x ; y_{\alpha}\right\rangle \rightarrow C\left\langle x ; y_{\alpha^{\prime}}\right\rangle$ o homomorfismo determinado pelas condições $\left.\eta_{\alpha}\right|_{C_{0}}=$ $\eta$ e $\eta_{\alpha}\left(y_{\alpha j}\right)=y_{\alpha^{\prime} j}$, para todo $j$. Então $\eta_{\alpha}$ é um isomorfismo, cujo inverso é dado pela aplicação $\eta_{\alpha}^{-1}$, definida de modo análogo, pondo $\eta^{-1}$ em lugar de $\eta$. Nos moldes do primeiro parágrafo, concluímos que $\eta_{\alpha}\left(I_{\alpha}^{0}\right) \subseteq I_{\alpha^{\prime}}^{0}$ e também que $\eta_{\alpha}^{-1}\left(I_{\alpha^{\prime}}^{0}\right) \subseteq I_{\alpha}^{0}$. Observemos agora que a aplicação de $\mathfrak{I}_{\alpha}(D)$ em $\mathfrak{I}_{\alpha^{\prime}}(D)$ dada por $\varphi \mapsto \varphi \eta_{\alpha}^{-1}$ é injetiva e, para qualquer $\psi$ em $\mathfrak{I}_{\alpha^{\prime}}(D)$, temos $\psi=\left(\psi \eta_{\alpha}\right) \eta_{\alpha}^{-1}$, e manifestamente $\psi \eta_{\alpha} \in \mathfrak{I}_{\alpha}(D)$. Sucede que $\mathfrak{I}_{\alpha^{\prime}}(D)=\mathfrak{I}_{\alpha}(D) \eta_{\alpha}^{-1} \neq \emptyset$. Logo $\alpha^{\prime} \in A$. 
Conforme as argumentações do início desta seção, $\eta_{\alpha}$ induz um isomorfismo $\hat{\eta}_{\alpha}$ de $R_{\alpha}$ em $R_{\alpha^{\prime}}$, dado por $\hat{\eta}_{\alpha}\left(p\left\langle x ; y_{\alpha}\right\rangle+I_{\alpha}(D)\right)=\eta_{\alpha}(p)+I_{\alpha^{\prime}}(D)$. Analogamente, $\eta_{\alpha}^{-1}$ induz um isomorfismo $\hat{\eta}_{\alpha}^{-1}$ de $R_{\alpha^{\prime}}$ em $R_{\alpha}$ e claramente $\hat{\eta}_{\alpha}^{-1} \hat{\eta}_{\alpha}=\hat{\eta}_{\alpha} \hat{\eta}_{\alpha}^{-1}=1$.

Definamos $\theta$ de $C_{D}(x)$ em si mesmo da seguinte forma: dado $\bar{p}$ em $C_{D}(x)$ qualquer, digamos que $\bar{p}=p\left\langle x ; y_{\alpha}\right\rangle+I_{\alpha}(D) \in R_{\alpha}$ para algum $\alpha$ em $A$. Fazemos $\theta(\bar{p})=\hat{\eta}_{\alpha}(\bar{p})$. Vamos, a seguir, mostrar que $\theta$ não depende de um $\alpha$ em particular.

Se também $\bar{p} \in R_{\beta}$, repetimos os argumentos e as notações usados na demonstração da afirmação 2 do lema 3.2.3: tomamos $R_{\gamma}$ que contém ambos $R_{\alpha}$ e $R_{\beta}$ e as inclusões canônicas destes em $R_{\gamma}$ serão denotadas por $\iota_{\alpha}$ e $\iota_{\beta}$, respectivamente. Sejam $R_{\beta^{\prime}}, \hat{\eta}_{\beta}, R_{\gamma^{\prime}}$ e $\hat{\eta}_{\gamma}$ nos moldes da construção que fizemos acima para $\alpha$. Necessariamente, $\hat{\eta}_{\gamma} \iota_{\alpha}=\hat{\eta}_{\alpha}$ e $\hat{\eta}_{\gamma} \iota_{\beta}=\hat{\eta}_{\beta}$, do que resulta

$$
\hat{\eta}_{\beta}(\bar{p})=\left(\hat{\eta}_{\gamma} \iota_{\beta}\right)(\bar{p})=\hat{\eta}_{\gamma}(\bar{p})=\left(\hat{\eta}_{\gamma} \iota_{\alpha}\right)(\bar{p})=\hat{\eta}_{\alpha}(\bar{p}) .
$$

Isto mostra que $\theta$ está bem definido e é claramente um homomorfismo cuja restrição à imagem de $C\langle x\rangle$ em $C_{D}(x)$ coincide com $\eta$. Podemos, também, definir $\theta^{-1}$ de $C_{D}(x)$ em si mesmo fazendo $\theta^{\prime}(\bar{p})=\hat{\eta}_{\alpha}^{\prime}(\bar{p})$ e o que expusemos até aqui também mostra que $\theta^{\prime}$ não depende de um $\alpha$ em particular. Resulta que $\theta^{\prime} \theta=\theta \theta^{\prime}=1$, ou seja, $\theta$ é um automorfismo de $C_{D}(x)$ que estende $\eta$.

Finalmente, como $C_{D}(x)$ é gerado pela imagem de $C\langle x\rangle$, no sentido descrito nas discussões acima, se $\vartheta$ é um automorfismo de $C_{D}(x)$ que estende $\eta$, então $\vartheta=\theta$. Isto encerra esta prova.

Vamos mostrar duas últimas propriedades do anel $C_{D}(x)$. Vejamos primeiro que este pode ser considerado como um anel de funções racionais em $D$, de maneira natural.

Teorema 3.2.10. $C_{D}(x)$ pode ser considerado como um anel de funções racionais em $D$, no seguinte sentido: dado $\bar{p}$ qualquer em $C_{D}(x)$, então existe um conjunto $\widetilde{\mathfrak{I}}_{\alpha}(D) \subseteq \Lambda$ não vazio e, para todo $\lambda \in \widetilde{\mathfrak{I}}_{\alpha}(D)$, está definido $\bar{p}(\lambda)$ em $D$. Ademais, se $\bar{p} \neq 0$, então existe um subconjunto não vazio $\widetilde{\mathfrak{I}}_{\gamma}(D)$ de $\widetilde{\mathfrak{I}}_{\alpha}(D)$ tal que $\bar{p}(\lambda) \neq 0$ e $[\bar{p}(\lambda)]^{-1}=\left(\bar{p}^{-1}\right)(\lambda)$, para qualquer $\lambda$ em $\widetilde{\mathfrak{I}}_{\gamma}(D)$.

Prova: Propositalmente, já adiantamos no enunciado o conjunto que servirá de domínio para $\bar{p}$ : existe $\alpha$ em $A$ tal que $\bar{p}=p\left\langle x ; y_{\alpha}\right\rangle+I_{\alpha}(D) \in R_{\alpha}$, com $\mathfrak{I}_{\alpha}(D) \neq \emptyset$. Tomamos a projeção $\widetilde{\mathfrak{I}}_{\alpha}(D)$ e, dado $\lambda=\varphi_{0}$ em $\widetilde{\mathfrak{I}}_{\alpha}(D)$, para um único $\varphi$ em $\mathfrak{I}_{\alpha}(D)$, fazemos $\bar{p}(\lambda)=$ $\varphi\left(p\left\langle x ; y_{\alpha}\right\rangle\right)$. Se $p\left\langle x ; y_{\alpha}\right\rangle \equiv q\left\langle x ; y_{\alpha}\right\rangle\left(\bmod I_{\alpha}(D)\right)$, então

$$
\bar{p}(\lambda)=\varphi(p)=\varphi(q)=\bar{q}(\lambda),
$$

mostrando que $\bar{p}(\lambda)$ está bem definida.

Se $\bar{p}=p\left\langle x ; y_{\alpha}\right\rangle+I_{\alpha}(D) \neq 0$, segue do lema 3.2 .3 que existe $\gamma$ em $A$ tal que $\bar{p}^{-1}=\bar{q}=q\left\langle x ; y_{\gamma}\right\rangle+I_{\gamma}(D) \in R_{\gamma}$ e, além disso, $R_{\alpha} \hookrightarrow R_{\gamma}$, do que decorre $\widetilde{\mathfrak{I}}_{\gamma}(D) \subseteq \widetilde{\mathfrak{I}}_{\alpha}(D)$. Então $p q \equiv 1\left(\bmod I_{\gamma}(D)\right)$, do que redunda $1=\varphi(p q)=\varphi(p) \varphi(q)=\bar{p}(\lambda) \bar{q}(\lambda)$, ou seja, $[\bar{p}(\lambda)]^{-1}=\bar{q}(\lambda)=\left(\bar{p}^{-1}\right)(\lambda)$ para todo $\lambda \in \widetilde{\mathfrak{I}}_{\gamma}(D)$, como queríamos.

Relacionamos o último teorema a algumas propriedades aritméticas, no seguinte 
Corolário 3.2.11. Dados $\bar{p}_{1}, \ldots, \bar{p}_{n}$ em $C_{D}(x)$ quaisquer, existe um conjunto não vazio $\widetilde{\mathfrak{I}}_{\alpha}(D) \subseteq \Lambda$ e, para todo $\lambda \in \widetilde{\mathfrak{I}}_{\alpha}(D)$, temos $\bar{p}_{i}(\lambda)+\bar{p}_{j}(\lambda)=\left(\bar{p}_{i}+\bar{p}_{j}\right)(\lambda)$ e $\bar{p}_{i}(\lambda) \bar{p}_{j}(\lambda)=$ $\left(\bar{p}_{i} \bar{p}_{j}\right)(\lambda)$, para quaisquer $i, j \in\{1, \ldots, n\}$.

Para encerrar o nosso texto, vamos mostrar que, se $D$ tem dimensão infinita sobre seu centro, então qualquer outro anel com divisão gerado pelos elementos de $C\langle x\rangle$ é imagem homomórfica de um subanel local de $C_{D}(x)$, fazendo sentido referir-se a este como o anel universal de frações de $C\langle x\rangle$.

Seja $D$ uma $C$-álgebra com divisão de dimensão infinita sobre seu centro. Formemos o anel com divisão $C_{D}(x)$. Como verificamos, este é um anel com divisão gerado a partir dos elementos da álgebra livre $C\langle x\rangle$, por meio das operações de adição, de multiplicação e tomando inversos. Suponhamos que $\mathfrak{D}$ seja um outro anel com divisão igualmente gerado por $C\langle x\rangle$. Observemos que, necessariamente, a dimensão de $\mathfrak{D}$ sobre seu centro é infinita, o que acarreta $C_{D}(x) \cong C_{\mathfrak{D}}(x)$. De agora em diante, usaremos a notação $C(x)$. Vamos mostrar que existem um subanel $\mathfrak{C} \hookrightarrow C(x)$ e um epimorfismo $\psi: \mathfrak{C} \rightarrow \mathfrak{D}$ que estende a inclusão de $C\langle x\rangle$ em $\mathfrak{D}$. Construiremos uma cadeia $C\langle x\rangle=C_{0} \subseteq C^{1} \subseteq C^{2} \subseteq \ldots$ de subanéis de $C(x)$ e sucessivas extensões de homomorfismos $\psi_{0}, \psi_{1}, \psi_{2}, \ldots$ de $C^{i}$ em $\mathfrak{D}$. Por fim, faremos $\mathfrak{C}=\bigcup C^{i}$ e $\psi: \mathfrak{C} \rightarrow \mathfrak{D}$ de modo que $\left.\psi\right|_{C^{i}}=\psi_{i}$.

Consideremos o homomorfismo $\psi_{0}: C_{0} \hookrightarrow \mathfrak{D}$ que embute $C\langle x\rangle$ em $\mathfrak{D}$. Para cada subconjunto finito de elementos não nulos $\left\{p_{\alpha 1}=p_{\alpha 1}\langle x\rangle, \ldots, p_{\alpha n_{\alpha}}=p_{\alpha n_{\alpha}}\langle x\rangle\right\} \subseteq C\langle x\rangle$, formemos a álgebra livre $C\left\langle x ; y_{\alpha}\right\rangle=C\left\langle x ; y_{\alpha 1}, \ldots, y_{\alpha n_{\alpha}}\right\rangle$ e o $Q$-ideal $I_{\alpha}^{0}=\left(q_{\alpha 1}, \ldots, q_{\alpha n_{\alpha}}\right)$ de $C\left\langle x ; y_{\alpha}\right\rangle$, em que $q_{\alpha j}=q_{\alpha j}\left\langle x ; y_{\alpha}\right\rangle=y_{\alpha j} p_{\alpha j}-1$, para todo $j$. Então o homomorfismo $\widetilde{\psi}_{1}^{\alpha}$ de $C\left\langle x ; y_{\alpha}\right\rangle$ em $\mathfrak{D}$ determinado pelas condições $\left.\widetilde{\psi}_{1}^{\alpha}\right|_{C_{0}}=\psi_{0}$ e $\widetilde{\psi}_{1}^{\alpha}\left(y_{\alpha j}\right)=\psi_{0}\left(p_{\alpha j}\right)^{-1}$ é admissível, do que resulta $\mathfrak{I}_{\alpha}(\mathfrak{D}) \neq \emptyset$, ou seja, $\alpha \in A$. Está bem definido, portanto, o homomorfismo $\psi_{1}^{\alpha}: R_{\alpha} \rightarrow \mathfrak{D}$ dado por $\psi_{1}^{\alpha}(\bar{r})=\widetilde{\psi}_{1}^{\alpha}(r)$, em que $r=r\left\langle x ; y_{\alpha}\right\rangle$ é um representante qualquer de $\bar{r}$.

Observemos que nada nos impede de repetir os argumentos do lema 3.2.3 (partes $\mathbf{1}$ e 2) para a coleção de todos os $R_{\alpha}$ 's obtidos nos moldes do parágrafo anterior e afirmar que eles estão dispostos em um conjunto direcionado. Em seguida, usamos o teorema 3.2.5 para concluir que a união de todos eles forma um subanel de $C(x)$ - o qual denotaremos por $C^{1}$ - que contém cada elemento não nulo de $C\langle x\rangle$ e seu respectivo inverso. Podemos, agora, estender $\psi_{0}$ a um homomorfismo $\psi_{1}: C^{1} \rightarrow \mathfrak{D}$, da seguinte maneira: para cada $\bar{r} \in C^{1}$, temos $\bar{r} \in R_{\alpha}$, para algum $R_{\alpha}$ na construção acima, e pomos $\psi_{1}(\bar{r})=\psi_{1}^{\alpha}(\bar{r})$. Os mesmos argumentos em 3.2.3 e em 3.2.5 mostram que $\psi_{1}$ não depende de um $\alpha$ em particular, estando, assim, bem definido. Manifestamente, $\psi_{1}$ é uma extensão de $\psi_{0}$.

Passamos para o segundo estágio: para cada subconjunto finito $\left\{\bar{r}_{\beta 1}, \ldots, \bar{r}_{\beta n_{\beta}}\right\}$ de $C^{1}-\operatorname{ker} \psi_{1}$, digamos $\bar{r}_{\beta j}=r_{\beta j}\left\langle x ; y_{\alpha}\right\rangle+I_{\alpha}(\mathfrak{D}) \in R_{\alpha}$, formamos a álgebra livre $C\left\langle x ; y_{\gamma}\right\rangle=$ $C\left\langle x ; y_{\alpha}, y_{\beta}\right\rangle$ e o ideal $I_{\gamma}^{0}=\left(q_{\alpha 1}, \ldots, q_{\alpha n_{\alpha}}, q_{\beta 1}, \ldots, q_{\beta n_{\beta}}\right)$, em que os $q_{\alpha i}$ 's são como antes e, para cada $j$ em $\left\{1, \ldots, n_{\beta}\right\}, q_{\beta j}=y_{\beta j} r_{\beta j}-1$. Então o homomorfismo $\widetilde{\psi}_{2}^{\gamma}$ de $C\left\langle x ; y_{\alpha}, y_{\beta}\right\rangle$ em $\mathfrak{D}$ determinado pelas condições $\left.\widetilde{\psi}_{2}^{\gamma}\right|_{C\left\langle x ; y_{\alpha}\right\rangle}=\widetilde{\psi}_{1}^{\alpha}$ e $\widetilde{\psi}_{2}^{\gamma}\left(y_{\beta j}\right)=\widetilde{\psi}_{1}^{\alpha}\left(r_{\beta j}\right)^{-1}$ é admissível, 
portanto $\mathfrak{I}_{\gamma}(\mathfrak{D}) \neq \emptyset$, ou seja, $\gamma \in A$. Está bem definido o homomorfismo $\psi_{2}^{\gamma}: R_{\gamma} \rightarrow \mathfrak{D}$ dado por $\psi_{2}^{\gamma}(\bar{s})=\widetilde{\psi}_{2}^{\gamma}(s)$, em que $s=s\left\langle x ; y_{\gamma}\right\rangle$ é um representante qualquer de $\bar{s}$. Podemos, pelos mesmos motivos do parágrafo anterior, afirmar que os $R_{\gamma}$ 's construídos neste segundo passo estão dispostos em um conjunto direcionado e que $C^{2}=\bigcup R_{\gamma}$ é um subanel de $C(x)$ que contém cada elemento de $C^{1}$ (essencialmente porque $R_{\alpha} \hookrightarrow R_{\gamma}$ ) e os inversos de todos aqueles cuja imagem por $\psi_{1}$ é não nula. Além disso, obtemos também $\psi_{2}$ : $C^{2} \rightarrow \mathfrak{D}$, uma extensão de $\psi_{1}$ construída às expensas dos $\psi_{2}^{\gamma}$ 's.

Procedendo indutivamente, obtemos, conforme anunciamos em princípio, o subanel $\mathfrak{C}=\bigcup C^{i} \hookrightarrow C(x)$ e o homomorfismo $\psi: \mathfrak{C} \rightarrow \mathfrak{D}$ tal que $\left.\psi\right|_{C^{i}}=\psi_{i}$. Redunda da nossa construção que $\mathfrak{C}-\operatorname{ker} \psi$ é, precisamente, o conjunto das unidades de $\mathfrak{C}$. Por conseguinte, este é um anel local, cujo único ideal maximal é $\operatorname{ker} \psi$. Então $\psi(\mathfrak{C}) \cong \mathfrak{C} / \operatorname{ker} \psi$, o anel com divisão de resíduos de $\mathfrak{C}$ que, em particular, contém uma cópia de $C\langle x\rangle$. Como $\mathfrak{D}$ é um corpo gerado por $C\langle x\rangle$, concluímos que $\mathfrak{D}=\mathfrak{C} / \operatorname{ker} \psi$, ou seja, $\psi$ é, de fato, um epimorfismo. 


\section{Referências Bibliográficas}

[AL50] S. A. Amitsur e J. Levitzki. Minimal identities for algebras. Proceedings of the American Mathematical Society, 1(4):449-463, 1950.

[Ami55] S. A. Amitsur. The T-ideals of the free ring. Journal of the London Mathematical Society, 1(4):470-475, 1955.

[Ami65] S. A. Amitsur. Generalized polynomial identities and pivotal monomials. Transactions of the American Mathematical Society, páginas 210-226, 1965.

[Ami66] S. A. Amitsur. Rational identities and applications to algebra and geometry. Journal of Algebra, 3(3):304-359, 1966.

[Ber70] G. M. Bergman. Skew fields of noncommutative rational functions, after Amitsur. Séminaire Schützenberger-Lentin-Nivat, Année 1969, 70, 1970.

[Ber76a] G. M. Bergman. Rational relations and rational identities in division rings I. Journal of Algebra, 43(1):252-266, 1976.

[Ber76b] G. M. Bergman. Rational relations and rational identities in division rings II. Journal of Algebra, 43(1):267-297, 1976.

[Coh95] P. M. Cohn. Skew Fields. Theory of General Division Rings. Cambridge University Press, Cambridge, 1995.

[Deh22] M. Dehn. Über die grundlagen der projektiven geometrie und allgemeine zahlsysteme. Em Festschrift David Hilbert zu Seinem Sechzigsten Geburtstag am 23. Januar 1922, páginas 184-194. Springer, 1922.

[Her71] I. N. Herstein. Noncommutative rings. Mathematical Association of America, 1971.

[Hua50] L. Hua. On the multiplicative group of a field. Acad. Sinica Science Record, $3(1-6): 2,1950$.

[Jac45] N. Jacobson. Structure theory for algebraic algebras of bounded degree. Annals of Mathematics, páginas 695-707, 1945.

[Jac85] N. Jacobson. Basic Algebra, volume 1. W. H. Freeman \& Company, 1985. 
[Jac89] N. Jacobson. Basic Algebra, volume 2. W. H. Freeman \& Company, 1989.

[Kap48] I. Kaplansky. Rings with a polynomial identity. Bull. Amer. Math. Soc., (6):575580, 061948.

[Kap72] I. Kaplansky. Fields and rings. University of Chicago Press, 1972.

[Kur41] A. Kurosch. Ringtheoretische probleme, die mit dem burnsideschen problem uber periodische gruppen in zusammenhang stehen. Izvestiya Rossiiskoi Akademii Nauk. Seriya Matematicheskaya, 5(3):233-240, 1941.

[Lam99] T. Y. Lam. Lectures on Modules and Rings. Springer-Verlag, New York, 1999.

[Lam01] T. Y. Lam. A First Course in Noncommutative Rings. Springer-Verlag, New York, segunda edição, 2001.

[Ore31] O. Ore. Linear equations in non-commutative fields. Annals of Mathematics, páginas 463-477, 1931.

[Pas11] D. S. Passman. The algebraic structure of group rings. Dover Publications, 2011.

[Row80] L. H. Rowen. Polynomial identities in ring theory, volume 84. Academic Press, 1980. 


\section{Índice Remissivo}

anel

com divisão topológico, 12

de frações, 3

total de frações, 3

base de um filtro, 14

condição de Ore, 2

conjunto

de Ore, 2

de denominadores, 2

multiplicativo, 1

domínio, 3

elemento regular, 3

filtro, 13

homomorfismo

admissível, 22

padrão, 49

identidade

de Hua, 21

racional, 22

localização, 2

Q-ideal, 22

ultra-filtro, 14

ultra-produto, 15 